Florida International University FIU Digital Commons

\title{
The Role of Agriculture and Food Consumption in Tropical Conservation
}

Brian Machovina

brianmachovina@gmail.com

DOI: $10.25148 /$ etd.FI15032154

Follow this and additional works at: https://digitalcommons.fiu.edu/etd

Part of the Agriculture Commons, and the Ecology and Evolutionary Biology Commons

\section{Recommended Citation}

Machovina, Brian, "The Role of Agriculture and Food Consumption in Tropical Conservation" (2015). FIU Electronic Theses and Dissertations. 1841.

https://digitalcommons.fiu.edu/etd/1841 


\section{FLORIDA INTERNATIONAL UNIVERSITY}

Miami, Florida

\section{THE ROLE OF AGRICULTURE AND FOOD CONSUMPTION IN TROPICAL CONSERVATION}

A dissertation submitted in partial fulfillment of

the requirements for the degree of

DOCTOR OF PHILOSOPHY

in

BIOLOGY

by

Brian L. Machovina

2015 
To: Dean Michael R. Heithuas

College of Arts and Sciences

This dissertation, written by Brian L. Machovina, and entitled The Role of Agriculture and Food Consumption in Tropical Conservation, having been approved in respect to style and intellectual content, is referred to you for judgment.

We have read this dissertation and recommend that it be approved.

$\begin{array}{r}\hline \text { Jennifer Gebelein } \\ \hline \text { Jennifer Richards } \\ \hline \text { Steve Oberbauer } \\ \hline \text { Pallab Mozumder } \\ \hline \text { Kenneth J. Feeley, Major Professor }\end{array}$

Date of Defense: March 18, 2015

The dissertation of Brian L. Machovina is approved.

Dean Michael C. Heithaus College of Arts and Sciences

Dean Lakshmi N. Reddi University Graduate School

Florida International University, 2015 


\section{ACKNOWLEDGMENTS}

I would like to thank my friends and lab mates for their help with my research efforts. I would also like to thank my dissertation committee members, Dr. Jennifer Richards, Dr. Jennifer Gebelein, Dr. Steve Oberbauer, and Dr. Pallab Mozumder for their support over the past several years. I would especially like to thank my major professor, Dr. Kenneth J. Feeley, for his encouragement, persistence, and enthusiasm supporting my research interests, wherever they may have taken me. I also owe great thanks to Florida International University, the Department of Biological Sciences, and the very long list of professors within its walls that have taught and inspired me during the more than a decade of my life spent studying there for my B.S., .M.S. and Ph.D degrees. I would like to thank my brother Lt. Col. Brett J. Machovina for his assistance with the many GIS obstacles I encountered during my research. I am also grateful for the support from Dole Food Co. Inc., their research team in Costa Rica, Ty Rozier of Elevated Horizons, and Jesse Bushong of Marcus UAV during my UAV research efforts.

I owe the greatest thanks to my wife for her patience and continued support of my desire to complete a doctorate degree started nearly 20 years ago. Thank you! 


\section{ABSTRACT OF THE DISSERTATION \\ THE ROLE OF AGRICULTURE AND FOOD CONSUMPTION \\ IN TROPICAL CONSERVATION \\ by}

Brian L. Machovina

Florida International University, 2015

Miami, Florida

Professor Kenneth J. Feeley, Major Professor

A growing human population, shifting human dietary habits, and climate change are negatively affecting global ecosystems on a massive scale. Expanding agricultural areas to feed a growing population drives extensive habitat loss, and climate change compounds stresses on both food security and ecosystems. Understanding the negative effects of human diet and climate change on agricultural and natural ecosystems provides a context within which potential technological and behavioral solutions can be proposed to help maximize conservation. The purpose of this research was to (1) examine the potential effects of climate change on the suitability of areas for commercial banana plantations in Latin America in the 2050s and how shifts in growing areas could affect protected areas; (2) test the ability of small unmanned aerial vehicles (UAVs) to map productivity of banana plantations as a potential tool for increasing yields and decreasing future plantation expansions; (3) project the effects on biodiversity of increasing rates of animal product consumption in developing megadiverse countries; and (4) estimate the capacity of global pasture biomass production and Fischer-Tropsch hydrocarbon synthesis (IGCC-FT) processing to meet electricity, gasoline and diesel needs. The 
results indicate that (1) the overall extent of areas suitable for conventional banana cultivation is predicted to decrease by $19 \%$ by 2050 because of a hotter and drier climate, but all current banana exporting countries are predicted to maintain some suitable areas with no effects on protected areas; (2) Spatial patterns of NDVI and ENDVI were significantly positively correlated with several metrics of fruit yield and quality, indicating that UAV systems can be used in banana plantations to map spatial patterns of fruit yield; (3) Livestock production is the single largest driver of habitat loss, and both livestock and feedstock production are increasing in developing biodiverse tropical countries. Reducing global animal product consumption should therefore be at the forefront of strategies aimed at reducing biodiversity loss; (4) Removing livestock from global pasture lands and instead utilizing the biomass production could produce enough energy to meet $100 \%$ of the electricity, gasoline, and diesel needs of over 40 countries with extensive grassland ecosystems, primarily in tropical developing countries. 


\section{TABLE OF CONTENTS}

CHAPTER

PAGE

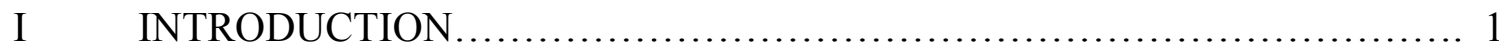

Literature Cited............................................................. 7

II CLIMATE CHANGE DRIVEN SHIFTS IN THE EXTENT AND

LOCATION OF AREAS SUITABLE FOR EXPORT BANANA



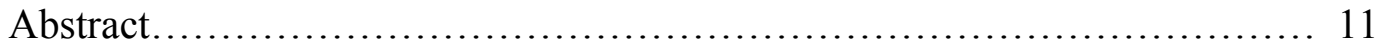

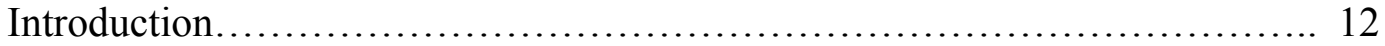

Methods........................................................................ 16

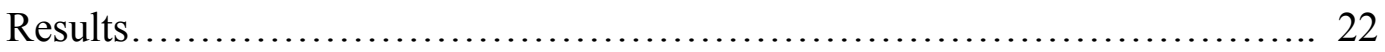

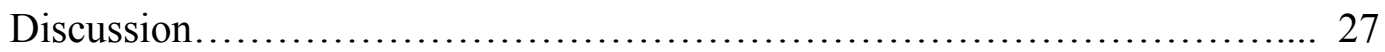

Literature Cited........................................................ 48

III AN EVALUATION OF UAV SYSTEMS FOR REMOTE SENSING OF BANANA PRODUCTION AND YIELD........................................................ 53

Abstract........................................................................ 54

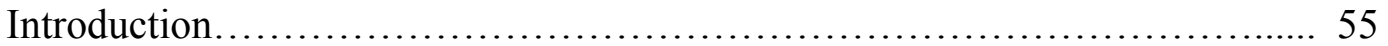



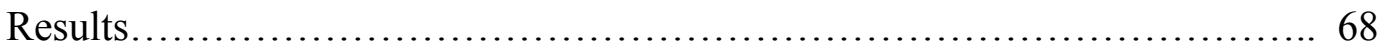

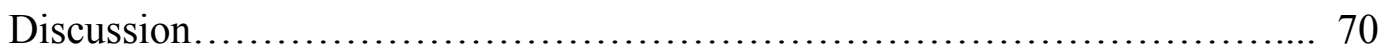

Acknowledgements..................................................... 87

Literature Cited.......................................................... 87

IV BIODIVERSITY CONSERVATION: THE KEY IS REDUCING HUMAN

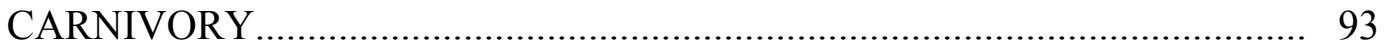

Abstract......................................................................... 94

Introduction........................................................ 94

Patterns of Biodiversity Loss Driven by Human Carnivory in the Tropics....... 96

Increasing Meat Production in Biodiverse Countries.......................... 98

The Importance of China.............................................. 102

Livestock-Driven Climate Change....................................... 105

The Important Role of Ruminants.......................................... 107

Human Health......................................................... 108

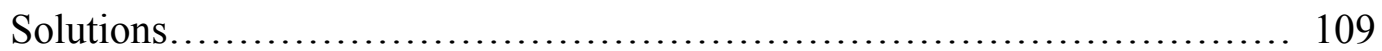



Literature Cited.................................................... 131

V POTENTIAL GLOBAL ENERGY AND BIOFUEL YIELDS FROM

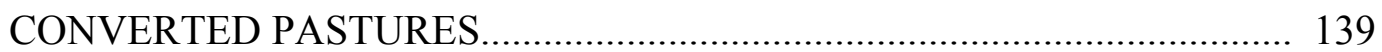

Abstract............................................................ 140

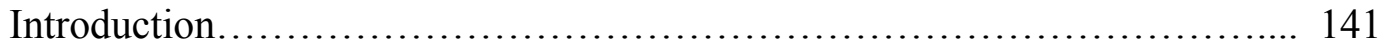


Methods.............................................................. 146

Results............................................................. 149

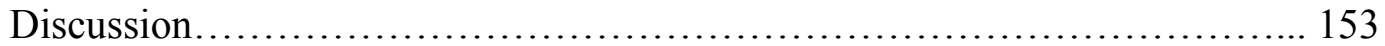

Literature Cited................................................. 178

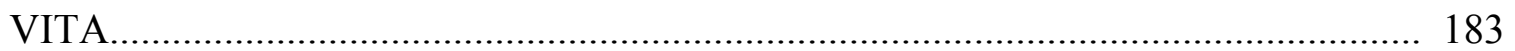




\section{LIST OF TABLES}

TABLE

PAGE

\section{CHAPTER II}

2.1. The climate envelope of the study area and areas suitable for conventional banana plantation cultivation

2.2. The current area $(\mathrm{km} 2)$ predicted suitable and not-suitable for conventional banana plantations, percent of country predicted suitable, actual area under banana cultivation (FAOSTAT, 2010), and percentage of suitable area under cultivation.

2.3. Climatic changes predicted by the model in (a) areas currently suitable for conventional banana plantation cultivation, (b) areas that lose suitability for cultivation, and (c) areas that gain suitability.

2.4. (a) Areas ( $\mathrm{km} 2)$ projected suitable under current conditions, each of the 7 GCMs, and the average of the 7 GCMs. (b) The number of models agreeing on the suitability of a location in the model, and the amount of area agreed upon by a majority ( 4 of 7 ) of the models

2.5. Changes in area $(\mathrm{km} 2)$ modeled as suitable for conventional banana plantations under current conditions and in 2060

2.6. Areas projected under current conditions and 2060 to be suitable for conventional banana plantation cultivation within protected areas....

2.7. Areas projected to be suitable for conventional banana plantation cultivation within land cover categories under current conditions and in 2060 .

2.8. The climate envelope of the study area and areas suitable for organic banana plantation cultivation

2.9. Changes in area $\left(\mathrm{km}^{2}\right)$ modeled as suitable for organic banana plantations under current conditions and in 2060

\section{CHAPTER V}

5.1. Potential energy production of global pastures by country, ranked highest to lowest by biomass production. The total area of harvestable pastures equals total area of $100 \mathrm{~km} 2$ cells with $\geq 25 \%$ pasture coverage. Harvestable biomass equals $50 \%$ of estimated ANPP. Ethanol is 
total produced via cellulosic methods $(0.255 \mathrm{~L} / \mathrm{kg}$ dry weight $)$ less energy required to harvest, transport and process biomass. Electricity, gasoline, and diesel are amounts co-produced via IGCC-FT processing less energy required to harvest, transport and process biomass. Total energy produced via IGCC-FT is $48.75 \%$ higher than cellulosic ethanol, and products are divided into electricity $(47 \%)$ and liquid fuels $(53 \%)$, the latter further divided into diesel $(53 \%)$ and gasoline (47\%). The ratios of potential electricity, gasoline, and diesel produced via IGCC-FT processing to their respective in-country demands.

5.2. The top 25 countries for harvestable area, harvestable biomass, and mean yield of biomass. Harvestable biomass is $50 \%$ of total ANPP, leaving $50 \%$ of production for habitat, soil building and carbon sequestration.

5.3. Potential energy production via IGCC-FT from croplands and pasture in the U.S. and Brazil. Electricity, gasoline, and diesel are amounts co-produced via IGCC-FT processing less energy required to harvest, transport and process biomass. Products are divided into electricity (47\%) and liquid fuels (53\%), the latter further divided into diesel (53\%) and gasoline (47\%). The ratios are of potential electricity, gasoline, and diesel produced via IGCC-FT processing to their respective in-country demands 


\section{LIST OF FIGURES}

FIGURE

PAGE

\section{CHAPTER II}

2.1. The locations of (a) sample sites and (b) areas predicted currently suitable for conventional banana plantations

2.2. Projected shifts in climate envelope variables (a) mean annual temperature, (b) maximum temperature of warmest month, (c) minimum temperature of coldest month, (d) annual precipitation, and (e) precipitation of the driest quarter for conventional banana plantation suitability. Solid black = currently suitable; dashed black = suitable now, suitable in 2060; solid gray $=$ unsuitable now, suitable in 2060 ; dashed gray $=$ suitable now, unsuitable in 2060 .

2.3. (a) Areas predicted to be suitable in 2060 for conventional banana plantations. (b) Net change from current suitability. For (b), green indicates areas projected to be become suitable in 2060, red indicates areas projected to lose suitability in 2060, and orange indicates no change in suitability from current conditions

2.4. The extent of land area in select Central and South American countries that is predicted to be suitable under current vs. future (2060) conditions for A) conventional and B) organic banana production. The diagonal line indicates the 1,1 relationship such that countries in the shaded areas are predicted to experience net decreases in the extent of suitable land area over the next 50 years due to climate change............................. 44

2.5. The locations of (a) sample sites and (b) areas predicted currently suitable for organic banana plantations.

2.6. Projected shifts in climate envelope variables (a) mean annual temperature, (b) maximum temperature of warmest month, (c) minimum temperature of coldest month, (d) annual precipitation, and (e) precipitation of the driest quarter for organic banana plantation suitability. Solid black = currently suitable; dashed black = suitable now, suitable in 2060; solid gray $=$ unsuitable now, suitable in 2060; dashed gray = suitable now, unsuitable in 2060

2.7. (a) Areas predicted to be suitable in 2060 for organic banana plantations.

(b) Net change from current suitability. For (b), green indicates areas projected to be become suitable in 2060, red indicates areas projected to lose suitability in 2060, and orange indicates no change in suitability from 
current conditions

\section{CHAPTER III}

3.1. (a) An elevation map indicating study area in relation to San Jose, the capital of Costa Rica, and (b) weekly mean rainfall beginning Jan. 1 (2008-2012) at the study area.

3.2. Rotary Wing UAV showing (a) approximate size and RC controller, (b) backpack antenna system for location tracking and flight control, and

(c) UAV during launch.................................................. 78

3.3. Fixed Wing UAV showing (a) approximate size, (b) antenna system for location tracking and live video capture, and (c) UAV mounted on launcher... 79

3.4. Example of (a) $627.5 \mathrm{~m} 2$ sample plot false-color image cropped from a raw image from the modified Canon SX260 camera and (b) its conversion into a scaled ENDVI image............................................ 80

3.5. ENDVI processed images of 20 random samples selected from harvest areas that supply cable lines. Green $=$ higher ENDVI values, yellow $=$ moderate ENDVI values, red = lower ENDVI values. Cable lines are ranked from lowest to highest mean ENDVI. Cables 15-44 are geographically located within close proximity (a single $\sim 1.3 \mathrm{~km} 2$ area), while cables 123-126 are located approximately $3 \mathrm{~km}$ away.

3.6. Mean ENDVI value ranked by cable line 82

3.7. Relationships among variables remotely sensed with modified Canon SX260 camera and banana production measures: (a) Mean ENDVI and Mean Bunch Weight; (b) Mean ENDVI and Mean Number of Hands per Bunch; (c) Mean ENDVI and Mean Banana Thickness on Second Hand; (d) Mean ENDVI and Mean Boxes Per Hectare; (e) Mean Bunch Weight and Mean Boxes Per Hectare; (f) Mean Plant Density and Mean Number of Hands per Bunch; (g) Mean Distance Between Plants and Mean Bunch Weight; (h) Mean Distance Between Plants and Mean Number of Hands Per Bunch; (i) Mean ENDVI and Mean Distance Between Plants.

3.8. Relationship between Mean ENDVI and Canopy Cover. Blue diamonds are sample plots from irrigated fields, red triangles are from non-irrigated......

3.9. (a) Locations of soil samples on mosaic of false-color imagery acquired with Tetracam Micro. Green = soil class I $(\mathrm{N}=14$, blue $=$ soil class II $(\mathrm{N}=12)$, yellow $=$ soil class III $(\mathrm{N}=12)$, red $=$ soil class IV $(\mathrm{N}=12)$.

(b) Locations of samples of NDVI values taken along cable lines $(\mathrm{N}=460)$. 
Green $=$ high NDVI levels, yellow $=$ moderate NDVI levels, red $=$ low

NDVI values......................................................... 85

3.10. Relationships among mean NDVI remotely sensed with Tetracam Micro and banana production measures: (a) Mean NDVI and Mean Bunch Weight; (b) Mean NDVI and Mean Number of Hands per Bunch; (c) Mean NDVI and Mean Banana Thickness on Second Hand; (d) Mean NDVI and Mean Length of Largest Finger; (e) Mean NDVI and Mean Boxes Per Hectare; (f) Mean NDVI and Mean Loss.................................... 86

\section{CHAPTER IV}

4.1. The diverse and interrelated negative effects of human carnivory on ecosystems, biodiversity, and human health. Climate change effects are highlighted in blue whereas direct ecosystem effects are highlighted in green. Bushmeat hunting directly reduces threatened animal populations which has negative cascading effects on ecosystems. Pasture creation is a powerful cause of deforestation and $\mathrm{CO} 2$ release. Grazing livestock compete with wild herbivores as well as instigate extirpation of predators by ranchers, both which cause cascading ecosystem effects. Grazing causes soil loss, downstream sedimentation of aquatic habitats, $\mathrm{CO} 2$ loss from soils, as well as enteric $\mathrm{CH} 4$ production. Intensive (industrial) livestock production releases $\mathrm{CH} 4$ and produces nutrient-rich wastes, which cause eutrophication of aquatic ecosystems as well as NO2 releases. The cultivation of feedcrops for intensive livestock production has many negative effects, primarily deforestation and habitat change that also releases $\mathrm{CO} 2$. The production of fertilizers releases $\mathrm{CO} 2$, and their excessive use releases $\mathrm{NO} 2$ and also causes eutrophication of aquatic habitats. Feedcrop cultivation competes for freshwater sources, extensively uses biocides, and causes large amounts of soil loss, all which negatively affect surrounding ecosystems. The consumption of animal products by humans is an underlying cause of the top causes of death of people, including heart disease (\#1 cause of death), strokes ( $\# 2$ cause of death), many cancers, and is also a contributor to the global rise of obesity and its associated ailments. International land and water grabbing for the production of feedcrops can cause smallholder agriculture to lose access to land and water, increasing local populations dependency on food aid and international food subsidies.

4.2. Human Trophic Level (HTL) of megadiverse countries based on consumption of livestock products in 1961 and 2009. An HTL of 2 indicates $0 \%$ of a nation's diet is composed of animal products whereas an HTL of 2.5 indicates $50 \%$ of a nation's diet composed of animal products. The blue line indicates the global median value of 2.21. Eleven of the 16 megadiverse countries represented here have increased HTLs 
from 1961 to 2009. (Data not available for Papua New Guinea, which is ranked among the 17 megadiverse countries). Consumption of bushmeat, especially important in Africa, was not included in analysis....

4.3. Map showing projected global increases of demand for meat (beef, pig, chicken) from 2000-2030. Legend indicates $\mathrm{kg} / \mathrm{km}$ demand increase. Developing countries of Latin America, Africa, and Asia exhibit the highest levels of demand increase. Data for Europe not available....

4.4. Projected increases in area required to produce meat in developing megadiverse (DMD) countries by 2050. (a)Extrapolating recent (1985-2012) production data for beef, chicken, and pork in each DMD country to 2050 (data for China shown) multiplied by (b) mean area required to produce livestock biomass provides (c) an estimate of area in each country required to produce livestock in 2050 as a percentage increase beyond total current agricultural area (2012). Agricultural area expansion needs can be met by internal expansion or by agricultural expansion in other countries and importation of feedcrops and/or meat products. This analysis addresses only beef, chicken, and pork. It does not include milk, eggs, other meat sources, or dairy, which would increase area projections.....

4.5. Developing megadiverse (DMD) countries with increasing rates of per capita meat (chicken, pork, beef) production. Eleven of 15 DMD countries have increasing rates of per capita meat production (chicken, pork, beef). The strong growth of China (highlighted) is of special note as its population ( 1.377 billion in 2012 ) is $59 \%$ larger than the combined populations (868 million) of the other $10 \mathrm{DMD}$ countries with increasing rates of meat production.

4.6. Animal product production in China 1961-2012. Production of animal products in China has increased dramatically, especially since the 1980s.

4.7. Production, exports and imports of soybeans for Brazil and China 1964-2012. Much of China's demand for feedcrops is driving deforestation and land-use change outside of its national boundaries, including within the Brazilian Amazon. Production of soy within China has increased minimally to meet growing Chinese demand for livestock feed, whereas imports have increased dramatically. Approximately half of Brazil's production is used internally, and half exported as feedcrops for markets around the world, including the European Union and China

4.8. Maps indicating density (high or low) of ruminants (cattle, goats, sheep) and species richness (high or low) of birds, mammals, and amphibians. Classification as 'high' indicate values above the mean value for all areas and 'low' indicate values below the mean value. Mean density 
value for ruminants $=5 / \mathrm{km} 2$. Mean species richness values $(\mathrm{spp} / 100 \mathrm{~km} 2)$

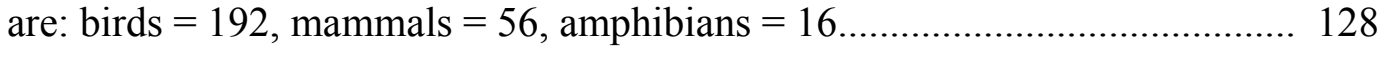

4.9. Average carbon equivalent footprint of meats and pulses per kilogram of product from a global meta-analysis of life-cycle assessment studies. Extensive beef involves cattle grazing across large pastoral systems, whereas intensive beef typically involves feedlots. Error bars represent standard errors.

\section{CHAPTER V}

5.1. Map of global distribution of pastures. Values are percent cover of $\sim 100 \mathrm{~km}^{2}$ cells.

5.2. Map of global NPP. Values are tons $\mathrm{C} / 100 \mathrm{~km} 2$. Maximum value is $128,549.8$ tons $\mathrm{C} / 100 \mathrm{~km}^{2}$. Mean value is $25,849.3$ tons $\mathrm{C} / 100 \mathrm{~km}^{2}$

5.3. Map of global distribution of harvestable pastures. (a) NPP of pastures. Values are tons $C / 100 \mathrm{~km}^{2}$. Maximum value is $118,455.7$ tons $C / 100 \mathrm{~km}^{2}$. Mean value is 165.3 tons $\mathrm{C} / 100 \mathrm{~km}^{2}$. (b) Locations of sample sites for comparison of Pasture Map NPP values to literature values.

5.4. Relationship between ANPP estimated from the map analysis and published ANPP values at 146 sample locations of pastures. The dotted line is the 1:1 ratio. ANPP is higher from literature values than map analysis at 104 locations ( $71 \%$ of the sites).

5.5. Potential energy output from pastures via IGCC-FT processing less energy required to harvest, transport and process biomass. (a) Total potential energy. Values are in billion megajoules. (b) Average yield. Values are in gigajoules/ha.

5.6. Ratios of potential energy production of pastures from IGCC-FT processing vs. internal demand for (a) electricity, (b) gasoline, and (c) diesel. 
CHAPTER I

INTRODUCTION 
Agriculture expansion driven by growing human population and shifting dietary habits is the primary driver of habitat loss, soil loss, and water use worldwide and a top cause of water pollution and global climate change (Steinfeld et al. 2006a). Over the 300 years ending in 1990, the extent of global cropland area increased more than five-fold and pasture areas increased more than six-fold, the latter encompassing an area 3.5 times larger than the United States. A direct cost of land being converted to food production was the loss of nearly one-half of all natural grasslands and the loss of nearly one-third of all natural forests worldwide (Goldewijk 2001). Although much of the habitat lost to agriculture in the 1800s was temperate forests and grasslands, the second half of the 1900s saw rapid agricultural expansion in tropical countries, predominantly at the expense of biodiverse tropical forests (Gibbs et al. 2010).

A human population projected to grow from a current 7 billion to 9 or more billion with improved economic capabilities will drive rising demand for more food and agricultural production (Alexandratos and Bruinsma 2012; Nellemann 2009). These demands can be met through expansion of agricultural lands, increases in yields, or shifts in dietary habits (Feeley and Machovina 2014; Godfray et al. 2010; Keyzer et al. 2005; Machovina and Feeley 2014b). Expansion of agricultural lands is the leading cause of natural habitat destruction (Foley et al. 2005; Foley et al. 2011) and future expansion needs have been estimated to require conversion of an additional 1 billion Ha of natural habitats during the first half of the $21^{\text {st }}$ century, an area approximately the size of the United States (Tilman et al. 2001). This expansion will cause large negative effects on biodiversity (Machovina and Feeley 2014c; Ripple et al. 2014a), soil loss (Steinfeld et al. 
2006a), fresh water supplies and water pollution (Foley et al. 2005), and global climate change (Fiala 2008; Garnett 2011; Ripple et al. 2014b).

Future agricultural production will depend on many complex factors. These include required increases in crop production to meet growing demand, increasing land scarcity, globalization (Lambin and Meyfroidt 2011), competing conservation needs, and global climate change (Fischer et al. 2005; Griggs and Noguer 2002; Iglesias et al. 2011). Concerns about the world's ability to feed itself that arose in the 1960s were quelled by widespread adoption of "green revolution" technology including the development of high-yielding varieties of wheat and rice, irrigation facilities, and the availability of inorganic fertilizers. However, since the 1990s, the rate of growth in food-grain production has been lower than the rate of population growth, and in order to feed future populations growth farm productivity must be increased with improved varieties combined with strategies for integrated nutrient management, integrated pest management, and efficient utilization of water and soil resources (Khush 1999) that also lower environmental damage. Information technology could be a key part of this strategy.

Climate change can potentially affect agriculture in many ways, for example by driving geographic shifts in the suitability and yields of key crop species (Jones and Thornton 2003; Tubiello et al. 2002) and varieties (White et al. 2006), as well as geographic shifts in the occurrence of the diseases and pests that affect crops (Júnior et al. 2008). If the potential effects of climate change are not accounted for through appropriate shifts in farming techniques and the distributions of where different crop species and varieties are planted, decreasing yields will lead to heightened risk of food insecurity for large portions of the global population (Nelson et al. 2009). Shifts in suitability of areas 
to grow crops could also affect natural ecosystems as societal pressure may increase to develop areas with increased suitability for crops.

Agricultural expansion is, by far, the leading cause of habitat destruction, including tropical deforestation (Geist and Lambin 2002), leading to the loss of the most biodiverse ecosystems on Earth. Current global rates of extinction are about 1000 times the estimated background rate of extinction, (Pimm et al. 2014) and the number of species in decline are much higher in the tropics - even after accounting for the greater species diversity of the tropics (Dirzo et al. 2014). Although some agricultural expansion is driven by farmers growing crops for direct human consumption, livestock production accounts for approximately three-quarters of all agricultural land and nearly one-third of the ice-free land surface of the planet, making it the single largest anthropogenic land use type (Steinfeld et al. 2006a). Livestock is also a primary cause of land degradation, pollution, climate change, overfishing, sedimentation of coastal areas, facilitation of invasions by alien species, and killing of wild predators (Steinfeld et al. 2006a). With livestock consuming $58 \%$ of directly used human-appropriated biomass globally (Krausmann et al. 2008) and one-third of global cereal production (Alexandratos and Bruinsma 2012; Foley et al. 2011), large amounts of energy are diverted into livestock via consumption of primary productivity of grasslands and croplands. Biomass consumed by livestock could potentially be utilized as a biomass-based fuel source if animal product consumption rates decreased. 
My Ph.D. research focused on four interrelated topics within the context of improving agricultural practices that address food security, biodiversity conservation, and climate change concerns. Technological solutions addressing these concerns are the focus of Chapters II and III, whereas Chapters IV and V address human behavioral choices (diet) that affect conservation and potential solutions for addressing climate change. In

\section{Chapter II, Climate Change Driven Shifts in The Extent and Location of Areas}

Suitable for Export Banana Production, I use species distribution modelling to predict the locations of areas that are currently suitable for commercial banana production in Central America and western South America, as well as the locations of areas that will be suitable for banana production in the future (2060) using climate change models. I focus on bananas as they are one of the most economically important food crops in the world, and they are of heightened conservation concern since they are grown exclusively in the tropics. In Chapter III, An Evaluation of UAV Systems for Remote Sensing of Banana Production and Yield, I compared the ease of use and efficiency of multi-rotor and fixed-wing UAV systems equipped with two different sensor systems for mapping spatial patterns of photosynthetic activity in banana plantations in Costa Rica. Spatial patterns of a photosynthetic indices based on reflected red/red edge and visible light (ENDVI) and reflected near infrared and red light (NDVI) were then compared to spatial patterns of physical soil quality, irrigation activity, and banana fruit production data in order to determine how well the remotely-sensed data can estimate banana production variables. A better understanding of the relationships between soil quality, water use, and other interacting variables of crop ecology that determine production could potentially lead to increases in yields. 
The global increase in per capita animal product consumption is perhaps the greatest threat to biodiversity and a key driver of climate change. In Chapter IV,

\section{Biodiversity Conservation: The Key Is Reducing Human Carnivory, I present a}

review of the connection between animal product consumption and current and likely future patterns of ecosystem degradation and biodiversity loss, the important influence of China in this relationship, the interwoven role of climate change, as well as the direct linkages with human health. In addition, I propose solutions for potentially reducing the negative effects of animal product consumption on ecosystems, biodiversity, and human health. Livestock production is not only a primary contributor to biodiversity loss, but its use of land is also an opportunity cost as it could potentially be used instead to develop carbon negative fuel sources that also improve soils and habitat. In Chapter V, Potential Global Energy and Biofuel Yields from Converted Pastures, I examined the potential of converting pastures and animal feedstock agricultural lands to LIHD biomass sources and the capacity of these systems to meet energy demands via cellulosic ethanol and Integrated Gasification Closed-Cycle Fischer-Tropsch (IGCC-FT) processing. The capacity of pastures to meet internal energy demands of countries via cellulosic ethanol and IGCC-FT processing was examined on a global basis, while conversion of feedstock agricultural lands to IGCC-FT biomass sources was also examined in the Unites States and Brazil, the two leading producers of biofuel and feedcrops. Large amounts of energy, far in excess of many country's internal demands for electricity, gasoline, and diesel, can potentially be produced from IGCC-FT processing of global pastures. 


\section{Literature Cited}

Alexandratos, N., \& Bruinsma, J. (2012) World agriculture towards 2030/2050: the 2012 revision. In. Rome: FAO

Dirzo, R., Young, H.S., Galetti, M., Ceballos, G., Isaac, N.J.B., \& Collen, B. (2014)

Defaunation in the Anthropocene. Science, 345, 401-406

Feeley, K.J., \& Machovina, B. (2014) Increasing preference for beef magnifies human impact on world's food web. Proceedings of the National Academy of Sciences

Fiala, N. (2008) Meeting the demand: An estimation of potential future greenhouse gas emissions from meat production. Ecological Economics, 67, 412-419

Fischer, G., Shah, M., Tubiello, F.N., \& Van Velhuizen, H. (2005) Socio-economic and climate change impacts on agriculture: an integrated assessment, 1990-2080.

Philosophical Transactions of the Royal Society B: Biological Sciences, 360, 2067-2083

Foley, J.A., DeFries, R., Asner, G.P., Barford, C., Bonan, G., Carpenter, S.R., Chapin, F.S., Coe, M.T., Daily, G.C., Gibbs, H.K., Helkowski, J.H., Holloway, T., Howard, E.A., Kucharik, C.J., Monfreda, C., Patz, J.A., Prentice, I.C., Ramankutty, N., \& Snyder, P.K. (2005) Global Consequences of Land Use. Science, 309, 570-574

Foley, J.A., Ramankutty, N., Brauman, K.A., Cassidy, E.S., Gerber, J.S., Johnston, M., Mueller, N.D., O/'Connell, C., Ray, D.K., West, P.C., Balzer, C., Bennett, E.M., Carpenter, S.R., Hill, J., Monfreda, C., Polasky, S., Rockstrom, J., Sheehan, J., Siebert, S., Tilman, D., \& Zaks, D.P.M. (2011) Solutions for a cultivated planet. Nature, 478, $337-342$

Garnett, T. (2011) Where are the best opportunities for reducing greenhouse gas emissions in the food system (including the food chain)? Food Policy, 36, Supplement 1, S23-S32

Geist, H.J., \& Lambin, E.F. (2002) Proximate Causes and Underlying Driving Forces of Tropical Deforestation. Bioscience, 52, 143-150

Gibbs, H.K., Ruesch, A.S., Achard, F., Clayton, M.K., Holmgren, P., Ramankutty, N., \& Foley, J.A. (2010) Tropical forests were the primary sources of new agricultural land in the 1980s and 1990s. Proceedings of the National Academy of Sciences, 107, 1673216737

Godfray, H.C.J., Beddington, J.R., Crute, I.R., Haddad, L., Lawrence, D., Muir, J.F., Pretty, J., Robinson, S., Thomas, S.M., \& Toulmin, C. (2010) Food Security: The Challenge of Feeding 9 Billion People. Science, 327, 812-818 
Goldewijk, K.K. (2001) Estimating global land use change over the past 300 years: The HYDE Database. Global Biogeochemical Cycles, 15, 417-433

Griggs, D.J., \& Noguer, M. (2002) Climate change 2001: the scientific basis.

Contribution of working group I to the third assessment report of the intergovernmental panel on climate change. Weather, 57, 267-269

Iglesias, A., Quiroga, S., \& Diz, A. (2011) Looking into the future of agriculture in a changing climate. European Review of Agricultural Economics, jbr037

Jones, P.G., \& Thornton, P.K. (2003) The potential impacts of climate change on maize production in Africa and Latin America in 2055. Global Environmental Change, 13, 5159

Júnior, J., Valadares Júnior, R., Cecílio, R.A., Moraes, W.B., Vale, F.X.R.d., Alves, F.R., \& Paul, P.A. (2008) Worldwide geographical distribution of Black Sigatoka for banana: predictions based on climate change models. Scientia Agricola, 65, 40-53

Keyzer, M.A., Merbis, M., Pavel, I., \& Van Wesenbeeck, C. (2005) Diet shifts towards meat and the effects on cereal use: can we feed the animals in 2030? Ecological Economics, 55, 187-202

Khush, G.S. (1999). Green revolution: preparing for the 21st century. Genome, 42, 646655

Krausmann, F., Erb, K.-H., Gingrich, S., Lauk, C., \& Haberl, H. (2008) Global patterns of socioeconomic biomass flows in the year 2000: A comprehensive assessment of supply, consumption and constraints. Ecological Economics, 65, 471-487

Lambin, E.F., \& Meyfroidt, P. (2011) Global land use change, economic globalization, and the looming land scarcity. Proceedings of the National Academy of Sciences, 108, 3465-3472

Machovina, B., \& Feeley, K.J. (2014a) Meat consumption as a key impact on tropical nature: a response to Laurance et. al. Trends in Ecology \& Evolution, 29, 430

Machovina, B., \& Feeley, K.J. (2014b) Taking a Bite Out of Biodiversity. Science, 343, 838

Nellemann, C. (2009) The Environmental Food Crisis: The Environment's Role in Averting Future Food Crises: a UNEP Rapid Response Assessment. UNEP/Earthprint

Nelson, G.C., Rosegrant, M.W., Koo, J., Robertson, R., Sulser, T., Zhu, T., Ringler, C., Msangi, S., Palazzo, A., \& Batka, M. (2009) Climate change: Impact on agriculture and costs of adaptation. Intl Food Policy Res Inst 
Pimm, S.L., Jenkins, C.N., Abell, R., Brooks, T.M., Gittleman, J.L., Joppa, L.N., Raven, P.H., Roberts, C.M., \& Sexton, J.O. (2014) The biodiversity of species and their rates of extinction, distribution, and protection. Science, 344

Ripple, W.J., Estes, J.A., Beschta, R.L., Wilmers, C.C., Ritchie, E.G., Hebblewhite, M., Berger, J., Elmhagen, B., Letnic, M., Nelson, M.P., Schmitz, O.J., Smith, D.W., Wallach, A.D., \& Wirsing, A.J. (2014a) Status and Ecological Effects of the World's Largest Carnivores. Science, 343

Ripple, W.J., Smith, P., Haberl, H., Montzka, S.A., McAlpine, C., \& Boucher, D.H. (2014b) Ruminants, climate change and climate policy. Nature Clim. Change, 4, 2-5

Steinfeld, H., Gerber, P., Wassenaar, T.D., Castel, V., \& Haan, C.d. (2006) Livestock's Long Shadow: Environmental Issues and Options. In. Rome: Food and Agriculture Organization of the United Nations

Tilman, D., Fargione, J., Wolff, B., D'Antonio, C., Dobson, A., Howarth, R., Schindler, D., Schlesinger, W.H., Simberloff, D., \& Swackhamer, D. (2001) Forecasting Agriculturally Driven Global Environmental Change. Science, 292, 281-284

Tubiello, F., Rosenzweig, C., Goldberg, R., Jagtap, S., \& Jones, J. (2002) Effects of climate change on US crop production: simulation results using two different GCM scenarios. Part I: Wheat, potato, maize, and citrus. Climate Research, 20, 259-270

White, M.A., Diffenbaugh, N., Jones, G.V., Pal, J., \& Giorgi, F. (2006) Extreme heat reduces and shifts United States premium wine production in the 21 st century. Proceedings of the National Academy of Sciences, 103, 11217-11222 


\section{CHAPTER II}

\section{CLIMATE CHANGE DRIVEN SHIFTS IN THE EXTENT AND \\ LOCATION OF AREAS SUITABLE FOR EXPORT BANANA PRODUCTION}

Machovina, B. \& K. Feeley (2013) Climate change driven shifts in the extent and

location of areas suitable for export banana production. Journal of Ecological Economics 95:83-95. 


\section{Abstract}

Demand for agricultural crops is projected to increase due to human population growth and increasing affluence. Compounding this challenge are the potential impacts of global climate change which may alter the suitability of areas for specific crop species and production methods. These shifts in suitability could have major impacts on local to national economies and potentially increase pressure to develop natural and protected areas. Here, I use the MAXENT Species Distribution Model (SDM) to map the locations of areas that are predicted to be suitable for commercial banana production in Central and northwestern South America. Using the downscaled climate projections for 2060 from seven leading global climate models I then predict the geographical shifts in areas suitable for banana production. I repeat this process for both conventional and organic banana production. Approximately half of the existing conventional plantations included in the analysis (57 of 117) are located in areas that are predicted to become unsuitable for banana production by 2060 . The overall extent of areas suitable for conventional banana cultivation is predicted to decrease by $19 \%$, but all countries are predicted to maintain some suitable areas. The extent of areas suitable for organic banana cultivation is predicted to nearly double due primarily to drying. Several countries (e.g., Colombia and Honduras) are predicted to experience large net decreases in the extent of areas suitable for banana cultivation; on the other hand, some countries (e.g., Mexico) are predicted to experience large net increases in the extent of suitable areas. The shifts in the location of areas that will be suitable for banana cultivation are predicted to occur mainly within areas outside of protected areas and that are already under agricultural production. As such, conservation concerns are minimal. These results, which map the shifting locations 
of areas suitable for commercial banana production, highlight how agriculture and industries can use species distribution modeling to proactively prepare for the future.

\section{Introduction}

Large expanses of lowland tropical forests have already been converted to agriculture (Wiley 2008), causing widespread losses of biodiversity and carbon stores (Brook et al. 2003; Turner 1996; Defries et al. 2002). Conversion of tropical forests to agriculture is ongoing, and is currently the leading driver of tropical deforestation and land conversion worldwide (Achard et al. 2002; Mayaux et al. 2005; Veldkamp et al. 1992). Due to increasing population sizes and affluence, the extent of land areas converted to agriculture is predicted to increase by approximately $18 \%$ by 2050 . This equates to a loss of one billion ha of natural habitats - an area larger than the United States - in less than 50 years (Tilman et al. 2001).

Future agricultural production will depend on many complex factors. These include required increases in crop production to meet growing demand, increasing land scarcity, globalization (Lambin \& Meyfriodt 2010), competing conservation needs, and global climate change (Iglesias et al., 2011; Fischer et al. 2005; IPCC 2001). Climate change can potentially affect agriculture in many ways, for example by driving geographic shifts in the suitability and yields of key crop species (Jones \& Thornton 2003; Tubiello et al. 2002) and varieties (White et al. 2006), as well as geographic shifts in the occurrence of the diseases and pests that affect crops (Cintra de Jesus et al. 2008). If the potential effects of climate change are not accounted for through appropriate shifts 
in farming techniques and the distributions of where different crop species and varieties are planted, decreasing yields will lead to heightened risk of food insecurity for large portions of the global population (Nelson et al. 2009). Although impacts of global climate change are expected to strongly affect the subsistence, or smallholder, farmers found predominantly in developing countries (Morton 2007), large-scale multinational agricultural industries will also be affected.

Adaptation of large-scale agricultural systems to climate change can potentially be addressed through a variety of strategies including the movement of crop production systems to follow suitable climatic conditions (Iglesias et al. 2011; Howden et al. 2007; Smit \& Skinner 2002). As such, it is essential that we develop models which can be used to predict how the locations and extents of areas suitable for the production of focal crop species will change under future climate change scenarios.

One tool that can potentially be used to help predict the locations of areas that will be suitable for the cultivation of specific crop species in the future is Species Distribution Models (SDMs). SDMs are a general suite of models that relate species' known occurrences to sets of environmental variables (e.g., mean annual temperature, annual precipitation, seasonality, slope, etc.)(Phillips et al. 2004; Pearson \& Dawson 2003). These relationships can then be interpolated and extrapolated across the broader landscape to produce maps of the species' predicted potential distributions (Anderson \& Martınez-Meyer 2004). Due to the power and relative ease of use, SDMs have become one of the most widely-used tools in conservation biology, biogeography and ecology 
(Franklin 2009; Richardson \& Whittaker 2010). For example, SDMs are used to generate predictions of where invasive species may occur under present climatic conditions (Giovanelli et al. 2008; Ficetola et al. 2007; Guisan \& Thuiller 2005; Peterson \& Vieglais 2001) as well as shifts in species' geographic distributions under future climatic change scenarios (Feeley and Silman 2010; Kearney et al. 2010; Hijman \& Grahams 2006). Despite their wide application, SDMs have rarely been applied to agricultural systems or crop species (Bradley et al. 2012; Trnka et al. 2007; Hijman \& Grahams 2006).

SDMs may appear to be limited in their ability to accurately predict the distributions of areas suitable for agricultural crop species since the climatic conditions on farms can be modified through practices such as irrigation, thereby enabling crop species to grow in areas that are unsuitable based on ambient climate alone (Jensen 2002; Wittwer \& Castilla 1995). For example, most crop species can theoretically be grown almost anywhere on the planet given sufficient environmental controls, such as externally supplied light, heat, irrigation, and soil amendments. However, these controls involve economic costs which are likely to increase in direct relation to the degree to which the natural ambient environment is unsuitable. Thus, while the potential distributions of many crop species are theoretically boundless, SDMs can be used to model the potential economically-viable distributions of crop species. For example, if a crop species is not currently grown in dry areas, then a safe assumption may be that it will not be economically viable to grow that species in similarly dry areas in the future even if it could potentially be grown there under intensive irrigation practices. Other variables that are important in defining economic limitations on production, such as distance to market 
and/or transportation centers, can also be explicitly included in SDMs as additional "environmental" variables, thereby potentially increasing the ability of SDMs to predict suitability of areas for crop production.

In this study, I use SDMs to predict the locations of areas that are and currently suitable for commercial banana production in Central America and western South America (Colombia, Ecuador, and Peru), as well as the locations of areas that will be suitable for banana production in the future (2060). I focus on bananas as they are one of the most economically important food crops in the world and are of heightened conservation concern since they are grown exclusively in the tropics (see discussion on bananas below). More specifically, I use the MAXENT SDM to produce current and future suitability maps for conventional banana plantation production as based on a sample of current plantation locations, select climatic and economic variables, and spatially explicit global climate change models (GCMs). I examine the predicted current and future suitability maps in relation to the distribution of different land cover classes and protected areas in order to investigate how climate change and food production needs may intersect with conservation priorities. Finally, a similar SDM analysis is performed to predict areas suitable for the production of organic bananas under current and future conditions. 


\section{Methods}

Bananas

Bananas (Musa acuminata Colla) are the developing world's fourth most valuable food crop, following only rice, wheat, and maize in terms of gross value of annual production (Frison et al. 2004), and are the $12^{\text {th }}$ most globally important plant crop by value and quantity (FAOSTAT 2010). Globally, over $100 \mathrm{MT}$ of bananas are grown annually on an estimated area of approximately 5 million ha, with production concentrated in Africa, Asia, India, the Caribbean, and Latin America (FAOSTAT 2010). Furthermore, bananas are a leading tropical agricultural export crop with export volumes of $>15$ MT per year and an annual export value of approximately $\$ 5$ billion per year. Nearly all of internationally traded bananas are the Cavendish variety (Robinson \& Sauco 2010). Indeed, for many decades, bananas have been the leading fresh fruit imported into the USA (Huang \& Huang 2007; FAO 2003a).

Overall, over $80 \%$ of banana exports come from Latin America where banana production is an important component of local and national economies (Evans \& Ballen 2012; Robinson \& Sauco 2010; FAO 2009). In 2010, Ecuador was the world's largest exporter of bananas, with an annual export production exceeding $5 \mathrm{MT}$ and $\$ 2$ billion in export value. This is nearly three times the quantity produced by Costa Rica, which ranks as the world's second largest exporter, followed by Colombia and the Philippines (FAOSTAT 2010). In contrast to most other export countries, farms in Ecuador are relatively small-scale; most farms are in the range of 10-50 ha and are owned and managed by local producers that sell to intermediaries or international companies (UNEP 
2002; Wunder 2001). In other Latin American countries, export bananas are produced primarily on large-scale plantations (some exceeding 2500 ha) that are controlled by private producers or large multinational companies (Robinson \& Sauco 2010; Wiley 2008).

Conventional export plantations use large amounts of nutrient inputs and agrochemicals to control diseases and pests. On average, almost $1 / 3$ of production costs in commercial plantations are allocated to fungicidal applications to control the leaf fungus Black Sigatoka (Micosphaerella fijiensis) which is considered to be the most damaging and costly threat to bananas (Marin et al. 2003). While organic banana production offers an alternative, less chemical intensive, production method, they currently account for only $\sim 1 \%$ of world trade (FAO 2003b) and approximately $3 \%$ of the total volume of fresh banana imports to the USA (Evans \& Ballen 2012). The Dominican Republic is the largest producer of organic bananas with an annual production of $\$ 200$ million in 2011 (elnuevodiario 2012), exceeding its conventional exports. This accounts for $40 \%$ of the global organic market volume (Frundt 2009; FAO 2003b), with $90 \%$ of the country's exports going to Europe (elnuevodiario 2012). The second largest global supplier of organic bananas is Ecuador, where output has grown at high rates (Evans \& Ballen 2012; FAO 2003b). Likewise, Peru is rapidly expanding its production of organic bananas: exports grew significantly between 2000 and 2007, in terms of both net value (from $\$ 264,000$ to $\$ 31$ million) and volume (from 856 tonnes to 64,586 tonnes) (COPLA 2009). Other major suppliers of organic bananas are Mexico, Colombia, Honduras, Guatemala and the Canary Islands (Spain) (FAO 2003b). Previous studies have suggested 
a high potential to increase organic production in several of these countries, and particularly in Mexico (Robinson \& Sauco 2010). For the US market, Ecuador, Colombia, and Peru are the leading suppliers. Between 2000 and 2010, organic banana imports to the USA grew from 27,000 tonnes to over 123,000 tonnes - a fourfold increase. Industry sources have reported that organic bananas represent one of the fastestgrowing commodities among organic produce (Evans and Ballen 2012).

\section{Species Distribution Modeling}

I visually identified 130 locations where conventional banana plantations currently occur by systematically scanning regional banana zones of Mexico, Guatemala, Belize, El Salvador, Honduras, Costa Rica, Panama, Colombia, Ecuador, and Peru in GoogleEarth. Monoculture banana plantations can be easily identified by sight in satellite images from the coloration and patterning of the vegetation. In order to confirm the accuracy and precision of my visual mapping in GoogleEarth, were verified the geographic coordinates of 20 banana plantations using maps published by the Dole Food Co.

I estimated the extent of areas potentially suitable for banana plantations under current conditions using the MAXENT species distribution model. MAXENT is an SDM based on machine learning and the principle of maximum entropy (Phillips et al. 2006; Phillips \& Dudik, 2008) and can be used to estimate 'the multivariate distribution of suitable habitat conditions (associated with species occurrences) in environmental feature-space' (Franklin, 2009). MAXENT is one of the most popular SDMs being used 
to estimate species ranges in relation to environmental predictors (Phillips \& Dudik 2008; Franklin 2009; Feeley \& Silman 2011) and has consistently performed well in model comparisons (Phillips \& Dudík, 2008; Elith et al. 2006). All models and calculations were performed at a resolution of 30 arc seconds (approximately $1 \mathrm{~km}^{2}$ at the equator); the 130 sample locations were reduced to 117 presences during aggregation into a pixel size of 30 arc seconds. Spatial analysis was performed in ArcGIS 10 (ESRI 2011) and R version 2.14.2 (R Development Core Team 2010).

Current suitability was modeled in MAXENT using five climatic variables (downloaded from the WorldClim database; www.worldclim) (Hijmans et al. 2005) that were hypothesized a priori to be affecting locations of banana cultivation. The climate variables used were (1) mean annual temperature, (2) annual precipitation, (3) the minimum temperature of the coldest month, (4) the maximum temperature of the warmest month, and (5) precipitation of driest quarter. Mean annual temperature and annual precipitation are commonly used variables in SDMs due to their strong relationship with the distributions of many species (Gentry 1988; Ter Steege et al. 2003; Kreft \& Jetz 2007). The minimum temperature of the coldest month and the maximum temperature of warmest month were included to account for the fact that bananas have known upper and lower temperature limits for fruit production (Turner \& Lahav 1983). Precipitation of the driest quarter was included as this variable likely limits the ability of bananas to be grown without irrigation and is likely a major factor determining the costs of production in areas where irrigation is required. 
In addition to the five climatic variables, I also included slope and distance to port. Plantations are selectively developed on flat terrain which generally has better soil quality and decreases costs of cultivation through ease of mechanization (Robinson \& Sauco 2010; Jones \& Mossison 1952). Distance to port was included to help incorporate costs of in-country transportation. Slope was calculated from Global 30 Arc-Second Elevation Dataset (GTOPO30) data sets downloaded from usgs.gov (U.S. Geological Survey's EROS Data Center 1996). Distance to port was calculated as the straight line distance from the nearest commercial port as listed on www.worldportsource.com.

Testing or validation was performed to assess the predictive performance of the MAXENT model. I randomly partitioned the sample points into 75\% 'training' and 25\% 'test' occurrences, creating a quasi-independent data for model testing (Guisan et al. 2003; Fielding \& Bell 1997). To identify the relative importance of the seven model variables to banana plantation cultivation suitability, I employed the permutation importance outcome of the MAXENT model, which has been shown to be a better measure of a variable's explanatory power than the percent contribution (Phillips 2011). The 117 presence points and seven variables were run with log output in MAXENT to define the current suitable locations for banana plantations, which are predicted on a map of continuous values from 0 to 1 , indicating the predicted probability that conditions are suitable for banana plantations (Phillips et al. 2006). I transformed this probability field to a binary map of the 'Suitable' versus 'Unsuitable' locations for banana plantations by thresholding: for each MAXENT run, I set the threshold as the cumulative probability at which the sum of sensitivity and specificity is maximized. In validation tests, this 
threshold criterion has been found to perform well and to have a high degree of accuracy in transforming probability fields to binary range maps (Jimènez-Valverde \& Lobo 2007).

Seven GCMs were used to estimate future climatic conditions in the study area: CGCM3.1(T47), MK3.0, IPSL-CM4, CCSM3, ECHAM5, HadCM3, and HAdGEM1. For each GCM, predictions of the 5 climate variables listed above for 2060 under the Alb emissions scenario were downloaded from www.ccafs-cliamte.org. Climate predictions were downscaled (delta method) to a resolution of 30 arc-seconds to match the current climate maps. I then used to the relationships between suitability and environment (5 climate variables $\&$ slope $\&$ distance to port) as identified by the MAXENT model to predict the distribution of areas that will be suitable for future banana production under the changes in climate predicted in each GCM (slope and distance to port were assumed to be static). The suitability maps for each GCM were thresholded as above and a single ensemble map of future suitability was generated based on majority consensus such that areas were classified as being 'suitable' if they were suitable in $\geq 4$ of the individual maps.

Maps of current and future predicted suitability distributions were compared to maps of legally protected areas and land cover classifications. A map of protected areas was downloaded from the World Database on Protected Areas (www.wdpa.org). Land cover classifications were downloaded from the USGS Global Land Cover Characterization (edc2.usgs.gov/glcc). 
A similar modeling of current and future suitability was performed for organic banana plantations using the published geographic coordinates of 33 existing organic plantations (Dole Food Co.) as sample points.

\section{Results}

Areas Currently Suitable for Conventional Banana Production

Based on the receiver-operation characteristic (ROC) curve and area under the curve (AUC) of the MAXENT model I determined that my application of the MAXENT model predicted conventional banana plantation occurrence with a high degree of accuracy (training data $\mathrm{AUC}=0.988$, test data $\mathrm{AUC}=0.990$ ). The variables ranked by permutation importance (contribution to model fit) from highest to lowest are: (1) distance to port, (2) slope, (3) mean annual temperature, (4) the minimum temperature of the coldest month, (5) the maximum temperature of the warmest month, (6) precipitation of the driest quarter, and (7) annual precipitation (Table 2.1). Distance to port contributed over $70 \%$ of the permutation importance, and adding slope, the two static economic variables combined contributed $87 \%$. Temperature variables contributed $10.4 \%$ and precipitation variables contributed the remaining $2.6 \%$.

Within the eleven-nation study area, a total of $228,209 \mathrm{~km}^{2}$ are predicted to be suitable for conventional banana production (Figure 2.1; Table 2.2) under current climatic conditions. Within the study region, $5,200 \mathrm{~km}^{2}$ are currently under cultivation (FAOSTAT 2010), comprising only $2.3 \%$ of the area predicted to be suitable. Colombia 
ranks as the country with the largest suitable area $\left(57,058 \mathrm{~km}^{2}\right)$ and Peru the smallest $\left(572 \mathrm{~km}^{2}\right)$. Ecuador, the number one banana exporter in Latin America, cultivates the largest portion of its suitable area (5.7\%), and Costa Rica and Colombia, which are the number two and three exporters, cultivate $2.7 \%$ and $1.4 \%$ of their suitable areas, respectively.

Predicted Changes in Climate of Currently Suitable Areas

In general, temperatures are predicted to increase and precipitation to decrease throughout the study area by 2060 (Table 2.3). In areas that are classified as being suitable under current conditions, mean annual temperature, the climatic variable with the highest permutation importance in the model, is predicted to increase from 26.2 to 28.9 ${ }^{\circ} \mathrm{C}(+10.2 \%)$ over the next 50 years, and the mean minimum temperature of the coldest month and the mean maximum temperature of the warmest month are predicted increase from 20.3 to $22.9^{\circ} \mathrm{C}(+12.8 \%)$ and 32.4 to $35.1^{\circ} \mathrm{C}(+8.3 \%)$, respectively. Overall, for currently suitable areas, levels of precipitation variables are projected to decrease, with mean precipitation of the driest quarter decreasing from an average of 154.3 to $128.7 \mathrm{~mm}$ $(-16.6 \%)$ and mean annual precipitation decreasing from an average of 2167.5 to 1739.8 $\mathrm{mm} \mathrm{yr}^{-1}(-19.7 \%)$. This overall increase in temperature and decrease in rainfall leads to loss of suitability in much of currently suitable areas. In general, areas that are predicted to become newly suitable by 2060 are cooler than those areas that are currently suitable (Figure 2.2). 


\section{Changes in Suitable Areas}

The extent of areas projected to be suitable for conventional banana plantation cultivation in 2060 under the seven GCMs ranges from $125,219 \mathrm{~km}^{2}$ to $234,346 \mathrm{~km}^{2}$, with an average of $188,731 \mathrm{~km}^{2}$ (Table $\left.2.4 \mathrm{a}\right)$. Areas where at least four of the seven models agree on suitability total $185,800 \mathrm{~km}^{2}$, a net decrease of $42,409 \mathrm{~km}^{2}(-18.6 \%)$ compared to the $228,209 \mathrm{~km}^{2}$ that are currently suitable (In Table $2.4 \mathrm{~b}$ ). Large areas projected to become unsuitable due to changes in climate are located in Colombia, while large areas projected to become newly suitable are located in Mexico (Figure 2.3). Of the 117 sample locations, 113 were modeled as being located in areas that are predicted to be suitable under current conditions (i.e., model sensitivity $=0.97$ ). Only 60 of the sample locations $(51.3 \%)$ are located in areas that are projected to still be suitable for plantation banana production in 2060 .

Eight of the eleven countries included in the study are projected experience net decreases in the extent of suitable land area by 2060 , with five countries projected to lose more than $50 \%$ of their suitable area. The largest loss of suitable area occurs in Colombia, with a projected loss of $35,352 \mathrm{~km}^{2}(-62 \%)$. The country that loses the largest proportion of suitable area is Guatemala (-74\%). Mexico is projected to experience the largest gain in suitable area, with a net increase of $44,396 \mathrm{~km}^{2}(+274 \%)$. Ecuador is also predicted to experience a net increase, with $6,439 \mathrm{~km}^{2}(+17 \%)$ more suitable area in 2060 (Table 2.5; Figure 2.4).

\section{Land Use}


Across the entire study area, only $37,336 \mathrm{~km}^{2}(5 \%)$ of the total area located within protected areas is predicted to be suitable for conventional banana plantation cultivation under current conditions (Table 2.6). By 2060 this amount is predicted to decrease by $34 \%$ to $24,628 \mathrm{~km}^{2}$ (3.3\% of total protected areas). The country with the largest portion of its protected areas suitable for banana production under current conditions is Nicaragua (33\%), followed by Panama (26\%) and El Salvador (24\%). In 2060, suitability for conventional banana plantation cultivation within protected areas is projected to decrease in eight of the eleven countries in the study. In the three countries with net increases in suitable areas, the absolute amounts are small, with the largest increase in suitable areas within protected areas occurring in Belize $\left(+509 \mathrm{~km}^{2}\right)$ (Table 2.6).

Under current conditions, areas suitable for conventional banana plantation production are primarily in the land cover classes of cultivated/cropland/managed (45\%) and tropical forests (38\%). Little shift in this allocation is predicted to occur by 2060 , with $44 \%$ of suitable areas being located in cultivated/cropland/managed land classes and $41 \%$ in tropical forests (Table 2.7).

\section{Organic Banana Cultivation}

As with conventional bananas, the receiver-operation characteristic (ROC) curve and area under the curve (AUC) indicate that my MAXENT model predicted organic banana plantation occurrence with a high degree of accuracy (training data AUC $=0.994$, test data $\mathrm{AUC}=0.950)$. The variables ranked by permutation importance (contribution to model fit) from highest to lowest are: (1) distance to port, (2) minimum temperature of 
coldest month, (3) annual precipitation, (4) precipitation of driest quarter, (5) maximum temperature of warmest month, (6) annual mean temperature, and (7) slope (Table 2.8). Distance to port contributed $58.9 \%$ of the permutation importance; slope, the other static economic variable, contributed the least $(0.2 \%)$. The second most import variable for organic cultivation, minimum temperature of the coldest month $(16.4 \%)$, had a mean value $\left(18.8^{\circ} \mathrm{C}\right)$ that was significantly lower than the same variable in the predicted conventional banana climate envelope $\left(20.3^{\circ} \mathrm{C}\right)$. The third most important variable for organic cultivation, annual precipitation $(14.3 \%)$, also had a mean value $(823.2 \mathrm{~mm})$ that was lower than the same variable in the predicted conventional banana climate envelope $(2167.5 \mathrm{~mm})$.

Under the current conditions a total of $147,742 \mathrm{~km}^{2}$ are classified as suitable for organic cultivation across all eleven countries. Due to increasing temperatures and decreasing rainfall, this amount is predicted to increase by a net of $128,988 \mathrm{~km}^{2}(+87 \%)$ by 2060 and eight of the eleven countries are predicted to increase in the extent of their suitable land area. Large expenses of areas that will become suitable are located in Mexico, Guatemala, and Honduras (Table 2.9; Figure 2.4), with the highest increase of area located in Mexico with $95,878 \mathrm{~km}^{2}$ additional suitable area $(+588 \%)$. The highest proportional increase is predicted for El Salvador $(+4608 \%)$ with $6,037 \mathrm{~km}^{2}$ additional suitable area. The largest loss of suitable area is predicted to occur in Colombia, with a net decrease of $42,849 \mathrm{~km}^{2}(-81 \%)$ in suitable land area.

\section{Discussion}


My species distribution models predict that climate change will cause large shifts in the locations and extents of areas that are suitable for either conventional or organic banana plantation production over the next 50 years. Although I predict that all of the study countries will maintain some suitable areas, several countries are predicted to experience large decreases in the extent of suitable land area. Compounding these losses, the location of areas that are suitable for production will shift even within national borders. Indeed, of the more than 100 existing conventional banana plantation sample locations included in my model, nearly half are located in areas that are predicted to become unsuitable in the near future.

Given the scale and importance of banana production and export in these countries, these losses and shifts in suitable land areas could have large effects on local and national economies. For example, Costa Rica and Colombia are now the 2nd \& 3rd most important banana export countries, respectively, but are each forecast to experience significant net declines in the extent of land areas suitable for conventional banana production. The 2011 value of banana exports for Costa Rica and Colombia were approximately $\$ 672$ million and $\$ 700$ million, respectively. Bananas are the most valuable agricultural export produced in Costa Rica and are the third-largest legal agricultural export of Colombia, behind coffee and flowers (FAOSTAT 2012). Colombia is predicted to be especially affected by climate change, with a predicted net decrease of $>60 \%$ in total area suitable for conventional banana production and a predicted net decrease of $>80 \%$ in area suitable for organic production. In contrast, Ecuador, which is now the top conventional and organic banana exporter, is predicted to experience an 
increase in the extent of suitable land area for both forms of production. Another potential "winner" is Mexico, which is predicted to experience very large increases in the extent of land areas suitable for conventional and especially organic production.

In my models, distance to port was the single most important variable in predicting the current distributions of both conventional and organic bananas. This highlights the power that economic costs, and in particular transportation costs, likely played in determining the locations of existing banana plantations. Another important variable in predicting the current distributions of conventional bananas was slope. This likely reflects the preferential development of plantations on the superior soils of flat alluvial zones as well as the large size and mechanized nature of many of these plantations. Steep slopes would prohibit the use of the networks of tower-supported cables as currently used by many of the most productive plantations to transport bananas from the plants to the packaging houses. In contrast to conventional bananas, slope was a much less important factor in predicting the distribution of organic plantations. This likely reflects the smaller scale and less mechanized nature of organic banana production.

The areas predicted to be suitable for conventional banana plantations have climates that are consistent with expectations based on independent assessments of banana productivity and climate (Robinson \& Sauco 2010). The reported overall optimal mean temperature for banana productivity (optimum balance between leaf emergence rate and net assimilation rate) is $27^{\circ} \mathrm{C}$ (Turner \& Lahav 1982) and the mean temperature of the areas predicted under my SDM to be suitable for banana production was $26.2^{\circ} \mathrm{C}$ for 
conventional and $25.6^{\circ} \mathrm{C}$ for organic. Another important climatic factor in predicting the distribution of both conventional and organic plantations was the temperature of the coldest month (average $=20.3^{\circ} \mathrm{C}$ for conventional and $18.8^{\circ} \mathrm{C}$ for organic). This is consistent with the known limitations of cold temperatures on banana growth, with a reported minimum mean temperature for development (leaf increase and leaf emergence rate) of $16^{\circ} \mathrm{C}$ (Turner \& Lahav 1982). Another prediction of my models that is consistent with known production methods/limitations is the fact that the average annual rainfall in areas predicted to be suitable for organic production is less than half that of the areas predicted to be suitable for conventional production. These differences in climates between areas suitable for conventional vs. organic production are due to the planting of organic bananas in areas more xeric areas. Dryer conditions decrease prevalence of fungal pathogens that would otherwise prohibit organic production. It should be noted, however, that these xeric locations require expensive irrigation to supply the high water requirements of bananas.

The loss of suitability for conventional banana production in my models was generally due to climates becoming too hot and too dry. In term of temperatures, areas that are predicted to be currently suitable but that will become unsuitable by 2060 have average mean annual temperatures that are projected to increase from 26.8 to $29.6^{\circ} \mathrm{C}$, average minimum temperatures of the coldest month that are projected to increase from 20.8 to $23.2{ }^{\circ} \mathrm{C}$, and average maximum temperature of warmest month that are predicted to increase from 33.2 to $36.1{ }^{\circ} \mathrm{C}$. Previous studies have indicated that bananas experience physiological heat stress at approximately $34^{\circ} \mathrm{C}$ and reduced net assimilation rates at 
mean annual temperatures approximately $31^{\circ} \mathrm{C}$ (Turner \& Lahav 1982). In terms of water availability, areas that are predicted to be currently suitable but that will become unsuitable over the next 50 years have average annual precipitations that are projected to decrease $22.6 \%$ and average precipitation of the driest quarter that will decrease $17.7 \%$.

It is important to keep in mind that areas that are predicted to become unsuitable in future may still be able to support commercial banana production under intensive management. Increasing temperatures may increase physiological stress and decrease yield of the widely grown Cavendish variety, and reduced rainfall may need to be offset with increased irrigation (Nelson et al. 2009). Management comes at a cost, and thus while it may be possible to maintain production, economic viability of these plantations could decrease to the point that these areas become unsuitable such that production is forced to shift to other more suitable areas where the future climate is better matched to optimal economic banana production. As such, one valuable outcome of this study is the prediction of not only where bananas can occur but also how costs of banana production may shift under changing climate. Despite technological advances such as improved crop varieties and irrigation systems, weather and climate are still key factors in agricultural productivity (Rosenzweig \& Iglesias 2001). Therefore meeting the low prices and varieties demanded by market forces may necessitate shifting cultivation to areas that have more optimal climate envelopes.

From a conservation standpoint, one positive outcome of my models is that large portions of the areas that are predicted to be suitable for banana production in the future 
are already under cropland. It may therefore be possible to maintain high levels of banana production with minimal forest loss by transitioning these areas into banana plantations. In many cases, the areas that are predicted to become suitable for banana production in the future are currently under less productive forms of agriculture, such as grazing (Cowan 1986), and thus the transition to banana production may result in net productivity gains.

Another positive outcome from a conservation standpoint is that there is little overlap between areas that are predicted to be suitable for banana production, either now or in the future, and legally protected areas. The relatively low suitability of protected areas for banana production is likely a relic of parks and protected areas being established on lands that were not valuable for agriculture, due either to the protection of commercial interests and/or the fact that many flat coastal alluvial zones had already been cleared for crop production prior to park establishment. 
Distance to Port (km)

Slope (deg.)

Annual Mean Temp. $\left({ }^{\circ} \mathrm{C}\right)$

Min. Temp. of Coldest Month $\left({ }^{\circ} \mathrm{C}\right)$

Max. Temp. of Warmest Month $\left({ }^{\circ} \mathrm{C}\right)$

Prec. of Driest Quarter (mm)

Annual Precipitation (mm)
Suitable Area

\section{Permutation}

$\frac{\text { Importance }}{71.2}$

15.8

4.2

3.5

2.7

1.5

1.1

\begin{tabular}{rrrr}
\multicolumn{4}{c}{ Suitable Area } \\
\hline Min & \multicolumn{1}{c}{ Max } & Mean & \multicolumn{1}{c}{ SD } \\
\hline 0 & 190.8 & 69.6 & 36.0 \\
0 & 5.2 & 0.5 & 0.5 \\
22.6 & 29.1 & 26.2 & 1.1 \\
12.7 & 23.9 & 20.3 & 12.6 \\
27.4 & 38.1 & 32.4 & 1.8 \\
0 & 1457.0 & 154.3 & 171.5 \\
190.0 & 7559.0 & 2167.5 & 1012.1
\end{tabular}

\begin{tabular}{rrrr}
\multicolumn{4}{c}{ Study Area } \\
\hline Min & \multicolumn{1}{c}{ Max } & Mean & \multicolumn{1}{c}{ SD } \\
\hline 0 & 1132.0 & 384.0 & 276.0 \\
0 & 58.7 & 2.8 & 4.0 \\
-7.6 & 29.5 & 21.4 & 5.8 \\
-20.2 & 23.9 & 11.9 & 8.5 \\
-0.6 & 42.7 & 30.5 & 5.6 \\
0 & 2495.0 & 165.9 & 206.6 \\
0 & 11314.0 & 1565.0 & 1142.4
\end{tabular}

Table 2.1. The climate envelope of the study area and areas suitable for conventional banana plantation cultivation. 


\begin{tabular}{|c|c|c|c|c|c|c|}
\hline & Suitable & $\begin{array}{c}\text { Not } \\
\text { Suitable }\end{array}$ & Total & $\begin{array}{c}\% \text { of } \\
\text { Country } \\
\text { Suitable }\end{array}$ & $\begin{array}{c}\text { Actual } \\
\text { Cultivated } \\
\text { Area }\end{array}$ & $\begin{array}{c}\% \text { of } \\
\text { Suitable } \\
\text { Area } \\
\text { Cultivated }\end{array}$ \\
\hline Mexico & 16,169 & $1,943,313$ & $1,959,481$ & $0.8 \%$ & 769.3 & $4.8 \%$ \\
\hline Belize & 3,324 & 18,641 & 21,965 & $15.1 \%$ & 27.0 & $0.8 \%$ \\
\hline Guatemala & 13,669 & 96,044 & 109,713 & $12.5 \%$ & 635.3 & $4.6 \%$ \\
\hline El Salvador & 2,436 & 18,102 & 20,538 & $11.9 \%$ & 27.0 & $1.1 \%$ \\
\hline Honduras & 11,850 & 100,874 & 112,724 & $10.5 \%$ & 254.5 & $2.1 \%$ \\
\hline Nicaragua & 46,674 & 81,988 & 128,663 & $36.3 \%$ & 7.5 & $0.0 \%$ \\
\hline Costa Rica & 16,157 & 34,920 & 51,078 & $31.6 \%$ & 429.0 & $2.7 \%$ \\
\hline Panama & 22,514 & 52,068 & 74,582 & $30.2 \%$ & 92.0 & $0.4 \%$ \\
\hline Colombia & 57,058 & $1,087,737$ & $1,144,794$ & $5.0 \%$ & 805.2 & $1.4 \%$ \\
\hline Ecuador & 37,778 & 219,486 & 257,264 & $14.7 \%$ & 2156.5 & $5.7 \%$ \\
\hline Peru & 572 & $1,298,363$ & $1,298,935$ & $0.0 \%$ & NA & NA \\
\hline Total & 228,201 & $4,951,536$ & $5,179,738$ & $4.4 \%$ & 5203.3 & $2.3 \%$ \\
\hline
\end{tabular}

Table 2.2. The current area $\left(\mathrm{km}^{2}\right)$ predicted suitable and not-suitable for conventional banana plantations, percent of country predicted suitable, the actual area under banana cultivation (FAOSTAT, 2010), and percentage of suitable area under cultivation. 
(a) Current Suitable Areas

Annual Mean Temp $\left({ }^{\circ} \mathrm{C}\right)$

Min Temp of Coldest Month $\left({ }^{\circ} \mathrm{C}\right)$

Max Temp of Warmest Month $\left({ }^{\circ} \mathrm{C}\right)$

Prec of Driest Quarter (mm)

Annual Precipitation (mm)

\begin{tabular}{rrrr}
\multicolumn{4}{c}{ Current } \\
\hline Min & Max & \multicolumn{1}{c}{ Mean } & \multicolumn{1}{c}{ SD } \\
\hline 22.6 & 29.1 & 26.2 & 1.07 \\
12.7 & 23.9 & 20.3 & 12.60 \\
27.4 & 38.1 & 32.4 & 17.72 \\
0 & 1457 & 154.3 & 171.50 \\
190 & 7559 & 2167.5 & 1012.10
\end{tabular}

\begin{tabular}{rrrrrrr}
\multicolumn{7}{c}{$2060 \mathrm{~s}$} \\
\hline 25.1 & $11.1 \%$ & 32 & $10.0 \%$ & 28.9 & $10.3 \%$ & 1.20 \\
15.1 & $18.9 \%$ & 26.5 & $10.9 \%$ & 22.9 & $12.8 \%$ & 1.30 \\
29.9 & $9.1 \%$ & 41.3 & $8.4 \%$ & 35.1 & $8.3 \%$ & 2.00 \\
0 & $0.0 \%$ & 1483 & $1.8 \%$ & 128.7 & - & 153.20 \\
156 & $-17.9 \%$ & 7825 & $3.5 \%$ & 1739.8 & $\ldots$ & 917.30
\end{tabular}

(b) Areas that Lose Suitability

Annual Mean Temp $\left({ }^{\circ} \mathrm{C}\right)$

Current

\begin{tabular}{rrrr}
\hline Min & Max & Mean & \multicolumn{1}{c}{ SD } \\
\hline 23.1 & 29.1 & 26.8 & 0.89 \\
12.7 & 23.9 & 20.8 & 1.29 \\
27.8 & 38.1 & 33.2 & 1.54 \\
0 & 1457 & 112.0 & 132.41 \\
190 & 7559 & 1997.1 & 919.64
\end{tabular}

\begin{tabular}{rrrrrrr}
\multicolumn{7}{c}{$2060 \mathrm{~s}$} \\
\hline \multicolumn{1}{c}{$\%$} & Max & \multicolumn{1}{c}{$\%$} & Mean & \multicolumn{1}{c}{$\%$} & \multicolumn{1}{c}{ SD } \\
\hline 25.7 & $11.3 \%$ & 32 & $10.0 \%$ & 29.6 & $10.4 \%$ & 0.97 \\
15.1 & $18.9 \%$ & 26.5 & $10.9 \%$ & 23.2 & $11.5 \%$ & 1.35 \\
30.3 & $9.0 \%$ & 41.3 & $8.4 \%$ & 36.1 & $8.7 \%$ & 1.69 \\
0.0 & $0.0 \%$ & 1483 & $1.8 \%$ & 92.1 & - & 116.57 \\
156.9 & $-17.4 \%$ & 7825 & $3.5 \%$ & 1545.8 & - & 846.91
\end{tabular}

(c) Areas that Gain Suitability

Annual Mean Temp $\left({ }^{\circ} \mathrm{C}\right)$

Min Temp of Coldest Month $\left({ }^{\circ} \mathrm{C}\right)$

Max Temp of Warmest Month $\left({ }^{\circ} \mathrm{C}\right)$

Prec of Driest Quarter (mm)

Annual Precipitation (mm)

\begin{tabular}{rrrr}
\multicolumn{4}{c}{ Current } \\
\hline Min & Max & \multicolumn{1}{c}{ Mean } & \multicolumn{1}{c}{ SD } \\
\hline 20.3 & 27.5 & 25.2 & 1.00 \\
12.4 & 23.1 & 17.7 & 1.87 \\
25.4 & 37.0 & 32.8 & 2.39 \\
1 & 1270 & 193.7 & 255.10 \\
146 & 7664 & 2077.4 & 1310.40
\end{tabular}

\begin{tabular}{rrrrrrr}
\multicolumn{7}{c}{$2060 \mathrm{~s}$} \\
\hline Min & \multicolumn{1}{c}{$\%$} & Max & \multicolumn{1}{c}{$\%$} & Mean & \multicolumn{1}{c}{$\%$} & \multicolumn{1}{c}{ SD } \\
\hline 23 & $13.3 \%$ & 30.4 & $10.5 \%$ & 28.1 & $11.5 \%$ & 1.09 \\
15 & $21.0 \%$ & 25.4 & $10.0 \%$ & 20.1 & $13.6 \%$ & 1.92 \\
28.1 & $10.6 \%$ & 40.1 & $8.4 \%$ & 35.9 & $9.5 \%$ & 2.65 \\
1 & $0.0 \%$ & 1269 & $-0.1 \%$ & 170.5 & - & 254.00 \\
172 & $17.8 \%$ & 8003 & $4.4 \%$ & 1780.4 & - & 1393.40
\end{tabular}

Table 2.3. Climatic changes predicted by the model in (a) areas currently suitable for conventional banana plantation cultivation, (b) areas that lose suitability for cultivation, and (c) areas that gain suitability. 
(a)

\begin{tabular}{lc} 
Current & $\begin{array}{c}\text { Suitable } \\
\text { Area } \\
\left(\mathrm{km}^{2}\right)\end{array}$ \\
CGCM3.1(T47) & $\begin{array}{c}228,201 \\
172,917\end{array}$ \\
MK3.0 & 202,510 \\
ISPL-CM4 & 206,147 \\
CCSM3 & 234,346 \\
ECHAM5 & 175,752 \\
HadCM3 & 125,219 \\
HadGEM1 & 204,225 \\
\cline { 2 - 2 } & \\
7 Model Ave & 188,731
\end{tabular}

(b)

\begin{tabular}{rc}
$\begin{array}{c}\text { No. of } \\
\text { Models } \\
\text { Agreeing }\end{array}$ & $\begin{array}{c}\text { Suitable } \\
\text { Area }\end{array}$ \\
1 & 48,305 \\
2 & 35,024 \\
3 & 35,018 \\
& \\
4 & 30,024 \\
5 & 35,395 \\
6 & 42,030 \\
7 & 78,351 \\
\cline { 2 - 2 } Majority & \\
$(4-7)$ & 185,800
\end{tabular}

Table 2.4. (a) Areas $\left(\mathrm{km}^{2}\right)$ projected suitable under current conditions, each of the $7 \mathrm{GCMs}$, and the average of the 7 GCMs. (b) The number of models agreeing on the suitability of a location in the model, and the amount of area agreed upon by a majority (4 of 7) of the models. 


\begin{tabular}{|c|c|c|c|c|c|c|c|c|c|}
\hline & $\begin{array}{c}\text { Current } \\
\text { Suitable } \\
\text { Area }\end{array}$ & $\begin{array}{l}\text { Addition } \\
\text { of } \\
\text { Suitable } \\
\text { Area }\end{array}$ & & $\begin{array}{c}\text { Loss of } \\
\text { Suitable } \\
\text { Area }\end{array}$ & & $\begin{array}{c}\text { No } \\
\text { Change in } \\
\text { Suitable } \\
\text { Area }\end{array}$ & & $\begin{array}{c}\text { Net } \\
\text { Change in } \\
\text { Suitable } \\
\text { Area }\end{array}$ & \\
\hline Mexico & 16,177 & 55,715 & $344 \%$ & $-11,319$ & $-70 \%$ & 4,854 & $30 \%$ & 44,396 & $274 \%$ \\
\hline Belize & 3,324 & 903 & $27 \%$ & -956 & $-29 \%$ & 2,368 & $71 \%$ & -53 & $-2 \%$ \\
\hline Guatemala & 13,669 & 2,357 & $17 \%$ & $-12,431$ & $-91 \%$ & 1,237 & $9 \%$ & $-10,074$ & $-74 \%$ \\
\hline El Salvador & 2,436 & 1,806 & $74 \%$ & $-2,336$ & $-96 \%$ & 99 & $4 \%$ & -530 & $-22 \%$ \\
\hline Honduras & 11,850 & 2,241 & $19 \%$ & $-8,319$ & $-70 \%$ & 3,531 & $30 \%$ & $-6,078$ & $-51 \%$ \\
\hline Nicaragua & 46,674 & 2,701 & $6 \%$ & $-22,355$ & $-48 \%$ & 21,506 & $46 \%$ & $-19,654$ & $-42 \%$ \\
\hline Costa Rica & 16,157 & 1,070 & $7 \%$ & $-10,012$ & $-62 \%$ & 6,145 & $38 \%$ & $-8,942$ & $-55 \%$ \\
\hline Panama & 22,514 & 2,454 & $11 \%$ & $-15,046$ & $-67 \%$ & 7,468 & $33 \%$ & $-12,592$ & $-56 \%$ \\
\hline Colombia & 57,058 & 12,192 & $21 \%$ & $-47,544$ & $-83 \%$ & 9,362 & $16 \%$ & $-35,352$ & $-62 \%$ \\
\hline Ecuador & 37,778 & 11,121 & $29 \%$ & $-4,681$ & $-12 \%$ & 33,097 & $88 \%$ & 6,439 & $17 \%$ \\
\hline Peru & 572 & 416 & $73 \%$ & -335 & $-59 \%$ & 237 & $41 \%$ & 82 & $14 \%$ \\
\hline Total & 228,209 & 92,977 & $41 \%$ & $-135,335$ & $-59 \%$ & 89,905 & $39 \%$ & $-42,358$ & $-19 \%$ \\
\hline
\end{tabular}

Table 2.5. Changes in area $\left(\mathrm{km}^{2}\right)$ modeled as suitable for conventional banana plantations under current conditions and in 2060. 


\begin{tabular}{|c|c|c|c|c|c|c|c|c|c|c|c|}
\hline & $\begin{array}{c}\text { Total } \\
\text { Protected } \\
\text { Areas }\end{array}$ & $\begin{array}{c}\text { Current } \\
\text { Suitable } \\
\text { Area } \\
\end{array}$ & & $\begin{array}{l}\text { Addition } \\
\text { of } \\
\text { Suitable } \\
\text { Area }\end{array}$ & & $\begin{array}{c}\text { Loss of } \\
\text { Suitable } \\
\text { Area }\end{array}$ & & $\begin{array}{c}\text { No } \\
\text { Change } \\
\text { in } \\
\text { Suitable } \\
\text { Area } \\
\end{array}$ & & $\begin{array}{c}\text { Net } \\
\text { Change } \\
\text { in } \\
\text { Suitable } \\
\text { Area } \\
\end{array}$ & \\
\hline Mexico & 204,993 & 1,791 & $1 \%$ & 1,179 & $66 \%$ & $-1,404$ & $-78 \%$ & 388 & $22 \%$ & -225 & $-13 \%$ \\
\hline El Salvador & 2,659 & 649 & $24 \%$ & 385 & $59 \%$ & -658 & $-101 \%$ & 2 & $0 \%$ & -273 & $-42 \%$ \\
\hline Peru & 184,035 & 56 & $0 \%$ & 317 & $570 \%$ & -18 & $-32 \%$ & 40 & $72 \%$ & 299 & $538 \%$ \\
\hline Belize & 8,698 & 1,040 & $12 \%$ & 731 & $70 \%$ & -222 & $-21 \%$ & 820 & $79 \%$ & 509 & $49 \%$ \\
\hline Colombia & 233,608 & 5,510 & $2 \%$ & 1,101 & $20 \%$ & $-4,886$ & $-89 \%$ & 675 & $12 \%$ & $-3,785$ & $-69 \%$ \\
\hline Honduras & 22,719 & 2,058 & $9 \%$ & 291 & $14 \%$ & $-1,907$ & $-93 \%$ & 186 & $9 \%$ & $-1,616$ & $-79 \%$ \\
\hline Guatemala & 33,708 & 1,469 & $4 \%$ & 738 & $50 \%$ & $-1,286$ & $-88 \%$ & 159 & $11 \%$ & -548 & $-37 \%$ \\
\hline Panama & 19,959 & 5,113 & $26 \%$ & 616 & $12 \%$ & $-3,304$ & $-65 \%$ & 1,840 & $36 \%$ & $-2,688$ & $-53 \%$ \\
\hline Costa Rica & 13,107 & 2,153 & $16 \%$ & 234 & $11 \%$ & $-1,249$ & $-58 \%$ & 913 & $42 \%$ & $-1,015$ & $-47 \%$ \\
\hline Ecuador & 45,616 & 1,760 & $4 \%$ & 629 & $36 \%$ & -142 & $-8 \%$ & 1,625 & $92 \%$ & 487 & $28 \%$ \\
\hline Nicaragua & 47,859 & 15,740 & $33 \%$ & 1,357 & $9 \%$ & $-5,210$ & $-33 \%$ & 10,247 & $65 \%$ & $-3,853$ & $-24 \%$ \\
\hline Total & 816,961 & 37,336 & $5 \%$ & 7,578 & $20 \%$ & $-20,286$ & $-54 \%$ & 16,895 & $45 \%$ & $-12,708$ & $-34 \%$ \\
\hline
\end{tabular}

Table 2.6. Areas projected under current conditions and 2060 to be suitable for conventional banana plantation cultivation within protected areas. 
Current Suitable Land Cover

\begin{tabular}{lrrrrrr} 
& $\begin{array}{c}\text { Tropical } \\
\text { Forest }\end{array}$ & $\begin{array}{c}\text { Other } \\
\text { Tree } \\
\text { Shrub }\end{array}$ & $\begin{array}{c}\text { Herba- } \\
\text { ceous }\end{array}$ & $\begin{array}{c}\text { Cultivated } \\
\text { Cropland } \\
\text { Managed }\end{array}$ & Other & Total \\
\cline { 2 - 7 } Mexico & 2,654 & 3,357 & 761 & 8,553 & 782 & 16,107 \\
El Salvador & 793 & 454 & 0 & 1,076 & 100 & 2,423 \\
Peru & 30 & 108 & 256 & 159 & 5 & 558 \\
Belize & 1,881 & 17 & 105 & 247 & 198 & 2,448 \\
Colombia & 8,619 & 1,977 & 8,838 & 33,447 & 784 & 53,665 \\
Honduras & 8,523 & 86 & 281 & 2,534 & 228 & 11,652 \\
Guatemala & 3,932 & 1,396 & 14 & 5,659 & 313 & 11,314 \\
Panama & 11,385 & 49 & 780 & 8,241 & 1,500 & 21,955 \\
Costa Rica & 9,180 & 575 & 59 & 3,856 & 441 & 14,111 \\
Ecuador & 4,590 & 1,887 & 5,138 & 21,826 & 896 & 34,337 \\
Nicaragua & 31,166 & 274 & 1,897 & 11,906 & 1,517 & 46,760 \\
Total & 82,753 & 10,180 & 18,129 & 97,504 & 6,764 & 215,330 \\
\% of Total & $38 \%$ & $5 \%$ & $8 \%$ & $45 \%$ & $3 \%$ &
\end{tabular}

Future Suitable Land Cover

\begin{tabular}{ccrrrr} 
Tropical & $\begin{array}{c}\text { Other } \\
\text { Tree } \\
\text { Forest }\end{array}$ & $\begin{array}{c}\text { Herba- } \\
\text { Shrub } \\
\text { ceous }\end{array}$ & $\begin{array}{c}\text { Cultivated } \\
\text { Cropland } \\
\text { Managed }\end{array}$ & $\begin{array}{c}\text { Other } \\
\text { Total }\end{array}$ \\
\hline 16,261 & 11,125 & 924 & 31,126 & 1,203 & 60,639 \\
197 & 591 & 0 & 897 & 157 & 1,842 \\
241 & 76 & 110 & 216 & 5 & 648 \\
2,749 & 11 & 44 & 282 & 234 & 3,320 \\
10,212 & 1,042 & 1,605 & 8,415 & 227 & 21,501 \\
4,066 & 16 & 148 & 1,510 & 38 & 5,778 \\
2,536 & 24 & 1 & 1,052 & 35 & 3,648 \\
6,501 & 36 & 317 & 2,505 & 455 & 9,814 \\
5,590 & 91 & 22 & 1,400 & 242 & 7,345 \\
5,728 & 2,325 & 6,171 & 29,125 & 880 & 44,229 \\
21,371 & 18 & 818 & 4,420 & 506 & 27,133 \\
\hline 75,452 & 15,355 & 10,160 & 80,948 & 3,982 & 185,897 \\
$41 \%$ & $8 \%$ & $5 \%$ & $44 \%$ & $2 \%$ &
\end{tabular}

$\%$ Change

\begin{tabular}{rrrrr} 
Tropical & $\begin{array}{c}\text { Other } \\
\text { Tree } \\
\text { Forest }\end{array}$ & $\begin{array}{c}\text { Herba- } \\
\text { Shrub } \\
\text { ceous }\end{array}$ & $\begin{array}{c}\text { Croplivated } \\
\text { Managed }\end{array}$ & Other \\
\hline $513 \%$ & $231 \%$ & $21 \%$ & $264 \%$ & $54 \%$ \\
$-75 \%$ & $30 \%$ & - & $-17 \%$ & $57 \%$ \\
$703 \%$ & $-30 \%$ & $-57 \%$ & $36 \%$ & $0 \%$ \\
$46 \%$ & $-35 \%$ & $-58 \%$ & $14 \%$ & $18 \%$ \\
$18 \%$ & $-47 \%$ & $-82 \%$ & $-75 \%$ & $-71 \%$ \\
$-52 \%$ & $-81 \%$ & $-47 \%$ & $-40 \%$ & $-83 \%$ \\
$-36 \%$ & $-98 \%$ & $-93 \%$ & $-81 \%$ & $-89 \%$ \\
$-43 \%$ & $-27 \%$ & $-59 \%$ & $-70 \%$ & $-70 \%$ \\
$-39 \%$ & $-84 \%$ & $-63 \%$ & $-64 \%$ & $-45 \%$ \\
$25 \%$ & $23 \%$ & $20 \%$ & $33 \%$ & $-2 \%$ \\
$-31 \%$ & $-93 \%$ & $-57 \%$ & $-63 \%$ & $-67 \%$ \\
\hline$-9 \%$ & $51 \%$ & $-44 \%$ & $-17 \%$ & $-41 \%$
\end{tabular}

Table 2.7. Areas projected to be suitable for conventional banana plantation cultivation within land cover categories under current conditions and in 2060. 
Suitable Area

Model Variable

Distance to Port $(\mathrm{km})$

Min. Temp. of Coldest Month

Annual Precipitation

Prec. of Driest Quarter

Max. Temp of Warmest Month

Annual Mean Temp

Slope

\begin{tabular}{rrrrr}
\hline $\begin{array}{c}\text { Permutation } \\
\text { Importance }\end{array}$ & \multicolumn{1}{c}{ Min } & Max & Mean & \multicolumn{1}{c}{ SD } \\
\hline 58.9 & 0 & 190.8 & 69.6 & 36 \\
16.4 & -2.5 & 23.9 & 18.8 & 3.66 \\
14.3 & 22 & 2201 & 823.2 & 501.04 \\
8 & 0 & 188 & 28.1 & 30.02 \\
1.3 & 19 & 40.4 & 32.6 & 2.61 \\
0.8 & 9.2 & 29.4 & 25.6 & 2.85 \\
0.2 & 0 & 5.2 & 0.5 & 0.5
\end{tabular}

Table 2.8. The climate envelope of the study area and areas suitable for organic banana plantation cultivation. 


\begin{tabular}{|c|c|c|c|c|c|c|c|c|c|}
\hline & $\begin{array}{c}\text { Current } \\
\text { Suitable } \\
\text { Area }\end{array}$ & $\begin{array}{l}\text { Addition } \\
\text { of } \\
\text { Suitable } \\
\text { Area }\end{array}$ & & $\begin{array}{c}\text { Loss of } \\
\text { Suitable } \\
\text { Area }\end{array}$ & & $\begin{array}{c}\text { No } \\
\text { Change in } \\
\text { Suitable } \\
\text { Area }\end{array}$ & & $\begin{array}{c}\text { Net } \\
\text { Change in } \\
\text { Suitable } \\
\text { Area }\end{array}$ & \\
\hline Mexico & 16,299 & 99,645 & $611 \%$ & $-3,767$ & $-23 \%$ & 12,532 & $77 \%$ & 95,878 & $588 \%$ \\
\hline Belize & 0 & 3,211 & - & & - & & - & 3,211 & - \\
\hline Guatemala & 847 & 23,122 & $2730 \%$ & -359 & $-42 \%$ & 488 & $58 \%$ & 22,763 & $2687 \%$ \\
\hline El Salvador & 131 & 6,160 & $4702 \%$ & -123 & $-94 \%$ & 8 & $6 \%$ & 6,037 & $4608 \%$ \\
\hline Honduras & 459 & 19,932 & $4342 \%$ & -430 & $-94 \%$ & 29 & $6 \%$ & 19,502 & $4249 \%$ \\
\hline Nicaragua & 6,987 & 12,389 & $177 \%$ & $-6,661$ & $-95 \%$ & 326 & $5 \%$ & 5,728 & $82 \%$ \\
\hline Costa Rica & 210 & 7,793 & $3711 \%$ & -191 & $-91 \%$ & 19 & $9 \%$ & 7,602 & $3620 \%$ \\
\hline Panama & 3,516 & 33 & $1 \%$ & $-3,396$ & $-97 \%$ & 0 & $0 \%$ & $-3,363$ & $-96 \%$ \\
\hline Colombia & 53,054 & 4,867 & $9 \%$ & $-47,716$ & $-90 \%$ & 5,338 & $10 \%$ & $-42,849$ & $-81 \%$ \\
\hline Ecuador & 34,596 & 4,658 & $13 \%$ & $-6,078$ & $-18 \%$ & 28,518 & $82 \%$ & $-1,420$ & $-4 \%$ \\
\hline Peru & 31,643 & 16,200 & $51 \%$ & -301 & $-1 \%$ & 31,342 & $99 \%$ & 15,899 & $50 \%$ \\
\hline Total & 147,742 & 198,010 & $134 \%$ & $-69,022$ & $-47 \%$ & 78,600 & $53 \%$ & 128,988 & $87 \%$ \\
\hline
\end{tabular}

Table 2.9. Changes in area $\left(\mathrm{km}^{2}\right)$ modeled as suitable for organic banana plantations under current conditions and in 2060 . 


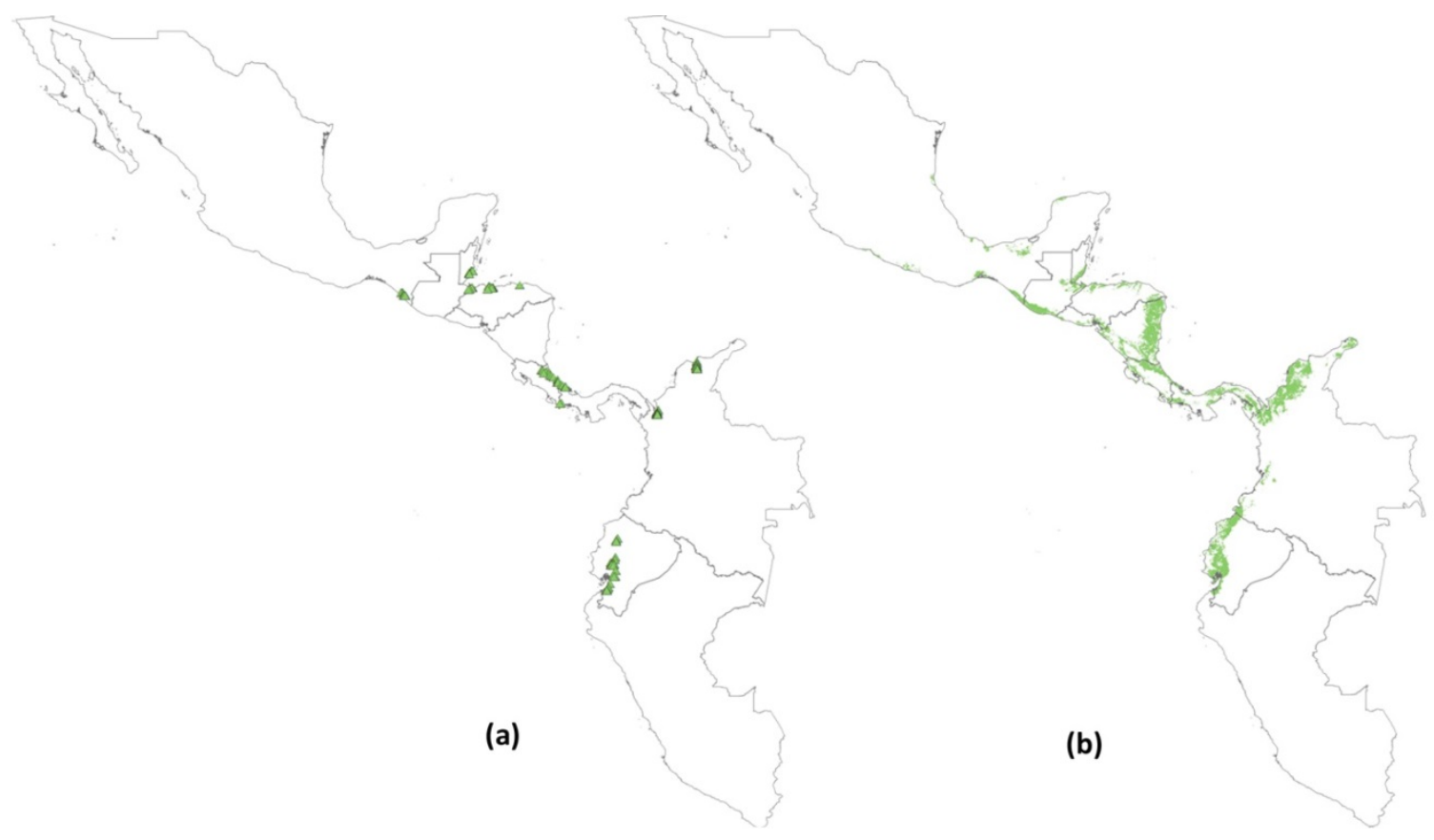

Figure 2.1. The locations of (a) sample sites and (b) areas predicted currently suitable for conventional banana plantations. 

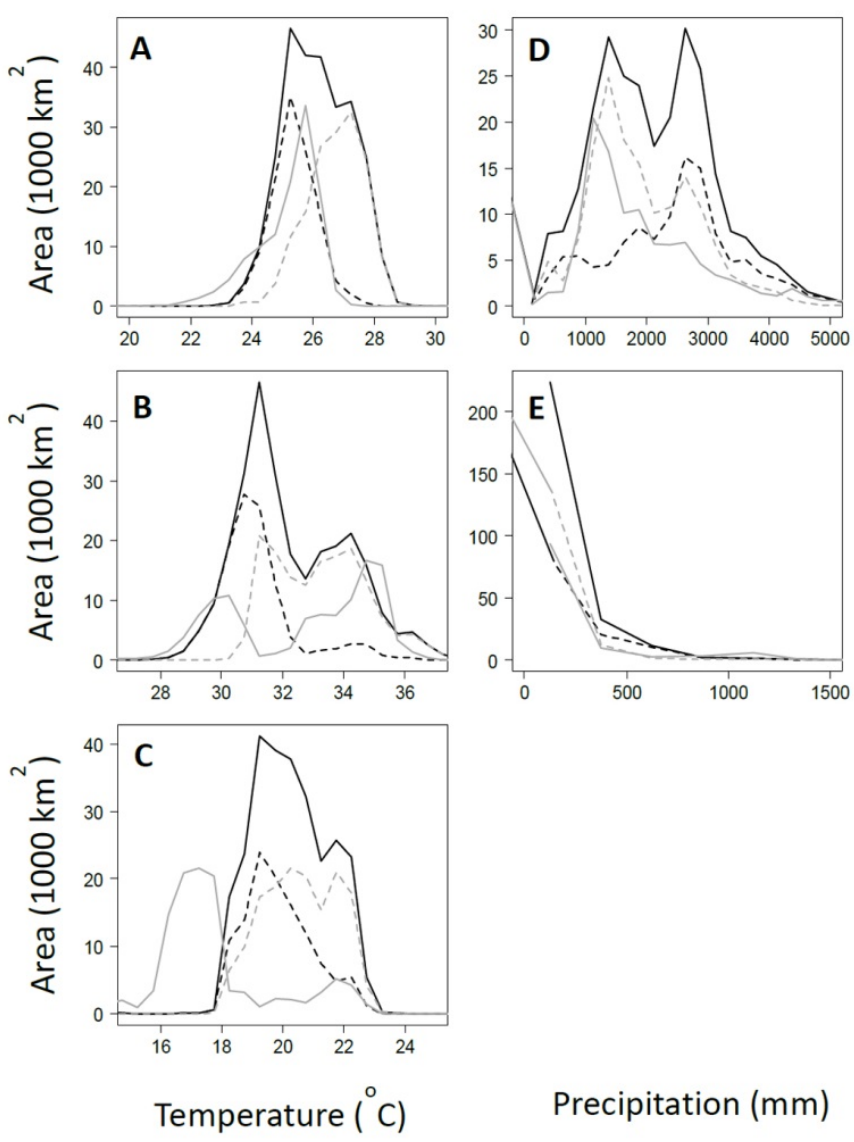

Precipitation ( $\mathrm{mm})$

Figure 2.2. Projected shifts in climate envelope variables (a) mean annual temperature, (b) maximum temperature of warmest month, (c) minimum temperature of coldest month, (d) annual precipitation, and (e) precipitation of the driest quarter for conventional banana plantation suitability. Solid black = currently suitable; dashed black $=$ suitable now, suitable in 2060; solid gray = unsuitable now, suitable in 2060; dashed gray = suitable now, unsuitable in 2060. 


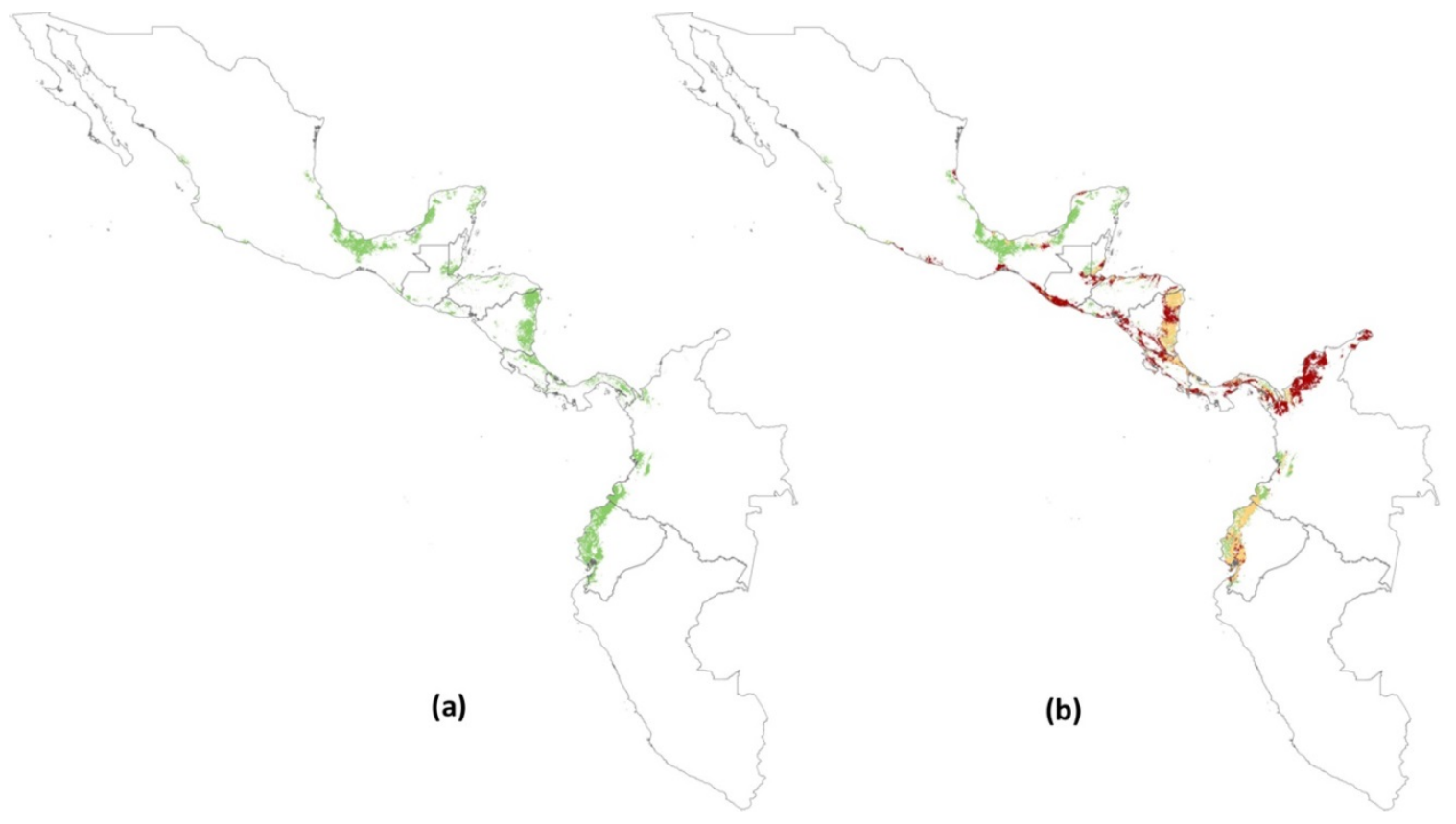

Figure 2.3. (a) Areas predicted to be suitable in 2060 for conventional banana plantations. (b) Net change from current suitability. For (b), green indicates areas projected to be become suitable in 2060, red indicates areas projected to lose suitability in 2060 , and orange indicates no change in suitability from current conditions. 



Figure 2.4. The extent of land area in select Central and South American countries that is predicted to be suitable under current vs. future (2060) conditions for (A) conventional and (B) organic banana production. The diagonal line indicates the 1:1 relationship such that countries in the shaded areas are predicted to experience net decreases in the extent of suitable land area over the next 50 years due to climate change. 




Figure 2.5. The locations of (a) sample sites and (b) areas predicted currently suitable for organic banana plantations. 

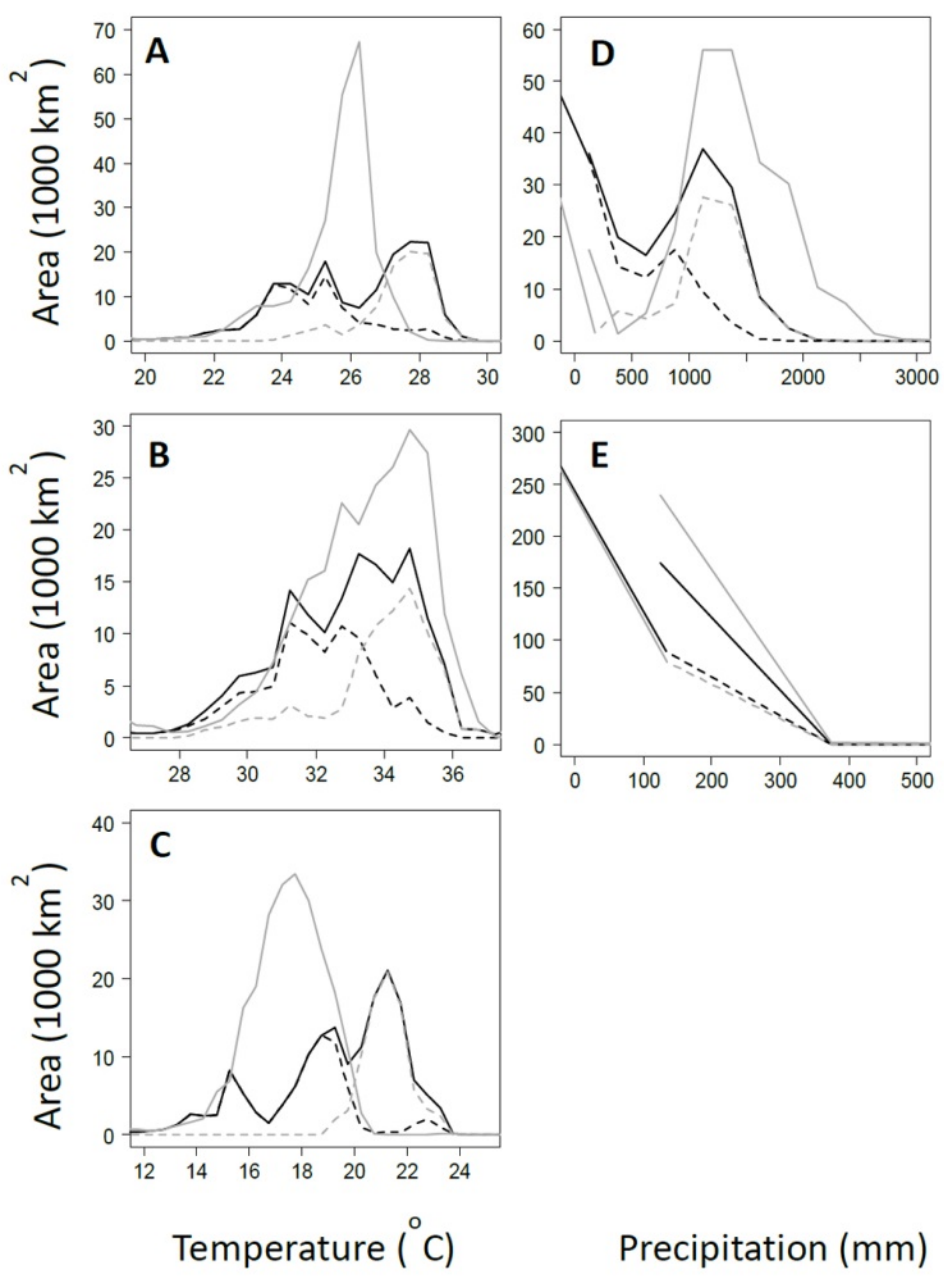

Figure 2.6. Projected shifts in climate envelope variables (a) mean annual temperature, (b) maximum temperature of warmest month, (c) minimum temperature of coldest month, (d) annual precipitation, and (e) precipitation of the driest quarter for organic banana plantation suitability. Solid black = currently suitable; dashed black = suitable now, suitable in 2060; solid gray $=$ unsuitable now, suitable in 2060; dashed gray $=$ suitable now, unsuitable in 2060. 


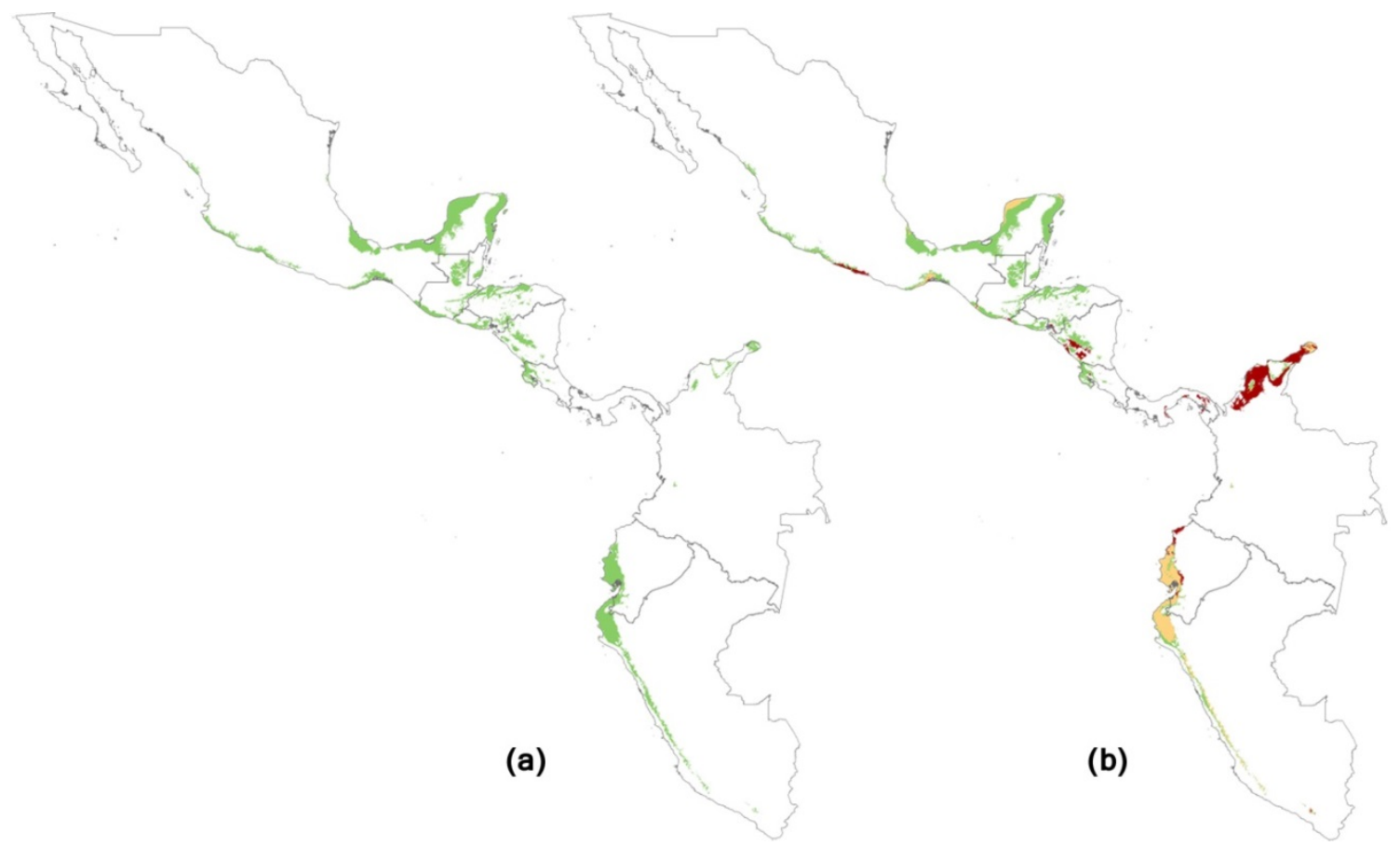

Figure 2.7. (a) Areas predicted to be suitable in 2060 for organic banana plantations. (b) Net change from current suitability. For (b), green indicates areas projected to be become suitable in 2060, red indicates areas projected to lose suitability in 2060, and orange indicates no change in suitability from current conditions. 


\section{Literature Cited}

Achard, F., H. Eva, H. Stibig, P, Mayaux, J. Gallego, T. Richards, J. Malingreau (2002)

Determination of deforestation rates of the world's humid tropical forests. Science 297 (5583), 999-1002

Anderson, R. P., \& E. Martınez-Meyer. (2004) Modeling species'geographic distributions for preliminary conservation assessments, an implementation with the spiny pocket mice (Heteromys) of Ecuador. Biological Conservation 116,167-179.

Bradley, B. A., L. D. Estes, D. G. Hole, S. Holness M. Oppenheimer, W. R. Turner, H. Beukes, R. E. Schulze, M. A. Tadross and D. S. Wilcove (2012) Predicting how adaptation to climate change could affect ecological conservation, secondary impacts of shifting agricultural suitability. Diversity and Distributions 18, 425-437.

Brook, B.W., N.S. Sodhi, and P. K. L. Ng (2003) Catastrophic extinctions follow deforestation in Singapore. Nature 424, 420-426.

Cintra de Jesus Jr., W., R. Valadares Jr., R.A. Cecílio, W. B. Moraes, F. X. Ribeiro do Vale, F.R. Alves, P. A. Paul (2008) Worldwide geographical distribution of Black Sigatoka for banana, predictions based on climate change models. Sci. Agric. (Piracicaba, Braz.) 65, 40-53.

COPLA (2009) Organic banana cultivation and fair trade in Peru. Comercio y Pobreza en Latino América Case Study.

Cowan, R.T. (1986) Beef and Dairy production in the wet tropics. 6th FAO Regional Training course. Pasture Management for the Wet Tropics of South East Asia Course Manual. pp. 193-197.

DeFries, R.S., R.A. Houghton, M.C. Hansen, C.B. Field, D. Skoll, and J. Townshend (2002) Carbon emissions from tropical deforestation and regrowth based on satellite observations for the 1980s and 1990s. PNAS 99(22), 14256-14261.

Elith, J., Graham, C.H., Anderson, R.P., Dudík, M., Ferrier, S., Guisan, A., Hijmans, R.J., Huettman, F., Leathwick, J.R., Lehmann, A., Li, J., Lohmann, L.G., Loiselle, B.A., Manion, G., Moritz, C., Nakamura, M., Nakazawa, Y., Overton, J.M., Peterson, A.T., Phillips, S.J., Richardson, K.S., Scachetti-Pereira, R., Schapire, R.E., Soberón, J., Williams, S., Wisz, M.S. \& Zimmermann, N.E. (2006) Novel methods improve prediction of species' distributions from occurrence data. Ecography 29, 129-151.

Elnuevodiario (2012) A raise in banana exports in the Dominican Republic, 8 March 2012. [online] URL: http://www.freshplaza.com/news detail.asp?id=93791. Accessed $2 / 13 / 2013$. 
ESRI (2011) ArcGIS Desktop, Release 10. Redlands, CA, Environmental Systems Research Institute.

Evans, E., and F. Ballen. (2012) Banana Market. EDIS FE901. Food and Resource Economics Department, Florida Cooperative Extension Service, Institute of Food and Agricultural Sciences, University of Florida, Gainesville.

FAO (2003a) The World Banana Economy 1985-2002. Food and Agriculture Organization of the United Nations.

FAO (2003b) Organic and fair trade bananas and the environmental and social certification in the banana sector. Food and Agriculture Organization of the United Nations. Committee on Commodity Problems, Intergovernmental Group on Bananas and Tropical Fruits. Puerto de la Cruz, Spain, 11-15 December 2003.

FAO (2009) Banana Statistics. Report to Members and Observers of the Sub-Group on Bananas of the Intergovernmental Group on Bananas and Tropical Fruits prepared by the Trade and Market Division, Food \& Agricultural Organization, Rome. [online] URL: http://www.fao.org/es/ESC/en. Accessed 2/13/13.

FAOSTAT (2010) FAO Database. Food and Agriculture Organization of the United Nations. [online] URL: http://www.faostat.fao.org. Accessed 2/13/13.

Feeley, K.J. \& Silman, M.R.(2010) Land-use and climate change effects on population size and extinction risk of Andean plants. Global Change Biology 16, 3215-3222.

Feeley, K.J. \& M.R. Silman (2011) Keep collecting, accurate species distribution modeling requires more collections than previously thought. Diversity and Distributions 17(6), 1132-1140.

Ficetola, G. F., W. Thuiller, C. Miaud (2007) Prediction and validation of the potential global distribution of a problematic alien invasive species - the American bullfrog. Diversity and Distributions 13(4), 476-485.

Fielding A.H. and Bell J.F. (1997) A review of methods for the assessment of prediction errors in conservation presence/absence models. Environ. Conserv. 24, 38-49.

Fischer, G., M. Shah, F. N. Tubiello and H. van Velhuizen (2005) Socio-economic and climate change impacts on agriculture: an integrated assessment, 1990-2080. Phil. Trans. R. Soc. B 360, 2067-2083.

Franklin, J. (2009) Mapping species distributions, spatial inference and predictions. Cambridge University Press, New York. 
Frison, E.A., J.V. Escalant, and S. Sharrock (2004) The global Musa genomic consortium: A boost for banana improvement. In Banana Improvement, Cellular, Molecular Biology, and Induced Mutations. Science Publishers, Inc.

Frundt, H.J. (2009) Fair bananas!: farmers, workers, and consumers strive to change an industry. University of Arizona Press.

Gentry, A.H. (1988) Changes in plant community diversity and floristic composition on environmental and geographic gradients. Annals of the Missouri Botanical Garden 75, 134 .

Giovanelli, J. G. R., C.F. B. Haddad, J.Alexandrino (2008) Predicting the potential distribution of the alien invasive American bullfrog (Lithobates catesbeianus) in Brazil. Biological Invasions 10(5), 585-590.

Guisan, A. and W. Thuiller (2005) Predicting species distribution: offering more than simple habitat models. Ecology Letters 8(9), 993-1009.

Haung, S. and K. Huang (2007) Increased U.S. Imports of Fresh Fruit and Vegetables. FTS-328-01. Economic Research Service/USDA.

Hijmans, R. J. and C.H. Grahams (2006) The ability of climate envelope models to predict the effect of climate change on species distributions. Global Change Biology $12(12), 2272-228$.

Hijmans, R.J., S.E. Cameron, J.L. Parra, P.G. Jones and A. Jarvis (2005) Very high resolution interpolated climate surfaces for global land areas. International Journal of Climatology 25, 1965-1978.

Howden M. S., Soussana J. F., Tubiello F. N., Chhetri N., Dunlop M., \& H. Meinke (2007) Adapting agriculture to climate change. Proceedings of the National Academy of Sciences 104(40), 19691-19696.

Iglesias, A., S. Quiroga, and A. Diz (2011) Looking into the future of agriculture in a changing climate. European Review of Agricultural Economics 38(3), 427-447.

Intergovernmental Panel on Climate Change (IPCC) (2001) Climate Change 2001, The Scientific Basis. Working Group 1 Contribution to the IPCC Third Assessment Report. [online] URL: www.ipcc.ch. Accessed 2/13/13.

Jensen, M.H. (2002) Controlled environment agriculture in deserts, tropics and temperate regions - a world review. ISHS Acta Horticulturae 578, 19-25. 
Jimènez-Valverde, A. \& J.M. Lobo (2007) Threshold criteria for conversion of probability of species presence to either-or presence-absence. Acta Oecologica 31, 361369.

Jones, C.F. and P.C. Morrison (1952) Evolution of the banana industry of Costa Rica. Economic Geography 28(1), 1-19.

Jones, P.G. and P.K. Thornton (2003) The potential impacts of climate change on maize production in Africa and Latin America in 2055. Global Environmental Change 13, 5159.

Kearney, M.R., B.A. Wintle, and W. P. Porter (2010) Correlative and mechanistic models of species distribution provide congruent forecasts under climate change. Conservation Letters 3(3), 203-213.

Kreft, H. \& W. Jetz, (2007) Global patterns and determinants of vascular plant diversity. Proceedings of the National Academy of Sciences 104, 5925-5930.

Lambin, E.F. and P. Meyfriodt (2010) Global land use change, economic globalization, and the looming land scarcity. PNAS 108(9), 3465-3472.

Marin, D.H., R.A. Romero, M. Guzman, T.B. Sutton (2003) Black Sigatoka: an increasing threat to banana cultivation. Plant Disease 87(3), 208-222.

Mayaux P., P. Holmgren, F. Achard, H. Eva, H. Stibig and A. Branthomme (2005) Tropical forest cover change in the 1990s and options for future monitoring. Philos Trans R Soc London Ser B 360, 373-384

Morton, J. (2007) The impact of climate change on smallholder and subsistence agriculture. Proceedings of the National Academy of Sciences 104(50), 19680-19685.

Nelson, G., M. Rosegrant, J. Koo, R. Robetson, T. Sulser, T. Zhu, C. Ringler, S. Msangi, A. Palazzo, M. Batka, M. Magalhaes, R. Valmonte-Santos, M. Ewing, and D. Lee (2009) Climate Change: Impact on Agriculture and Costs of Adaptation. Food Policy Report. International Food Policy Research Institute.

Pearson, R.G. and T.P. Dawson (2003) Predicting the impacts of climate change on the distribution of species: are bioclimate envelope models useful? Global Ecology and Biogeography 12(5), 361-371.

Peterson, A.T. and D. A. Vieglais (2001) Predicting species invasions using ecological niche modeling: new approaches from bioinformatics attack a pressing problem. BioScience 51(5), 363-371. 
Phillips, S. (2011) A Brief Tutorial on Maxent. [online] URL:

http://www.cs.princeton.edu/ schapire/maxent/tutorial/tutorial.doc. Accessed 2/13/2013.

Phillips, S. J. and M. Dudik (2008) Modeling of species distributions with Maxent: new extensions and a comprehensive evaluation. Ecography 31(2), 161-175.

Phillips, S. J., Dudik, M. and Schapire, R. E. (2004) A maximum entropy approach to species distribution modeling. In, Proc. of the 21st International Conference on Machine Learning, Banff, Canada, 2004.

Phillips, S.J., Anderson, R.P. \& Schapire, R.E. (2006) Maximum entropy modeling of species geographic distributions. Ecological Modeling 190, 231-259.

R Development Core Team (2010) R: A language and environment for statistical computing. R Foundation for Statistical Computing, Vienna, Austria. ISBN 3-900051-070, [online] URL: http://www.R-project.org/.

Richardson, D.M. \& R.J. Whittaker (2010) Conservation biogeography - foundations, concepts and challenges. Diversity and Distributions 16, 313-320.

Robinson, J. and V. Sauco (2010) Bananas and Plantains. CABI International, Oxfordshire, UK.

Rosenzweig, C. and A. Iglesias (2001) Potential impacts of climate change on world food supply. Data sets from a major crop modeling study. [online] URL: http://beta.sedac.ciesin.columbia.edu/giss crop study/index.html. Accessed 2/13/13.

Smit, B. and M. Skinner (2002) Adaptation options in agriculture to climate change: a typology. Mitigation and Adaptation Strategies for Global Change 7, 85-114.

Ter Steege, H., Pitman, N., \& Sabatier, D. (2003) A spatial model of tree alpha-diversity and tree density for the Amazon. Biodiversity and Conservation 12, 2255-2277.

Tilman, D. et al. (2001) Forecasting agriculturally driven global environmental change. Science 292, 281-284.

Trnka, M., F. Muškab, D. Semerádováa, M. Dubrovskýc, E. Kocmánkováa, Z. Žalud (2007) European Corn Borer life stage model, Regional estimates of pest development and spatial distribution under present and future climate. Ecological Modeling 207(2-4), $61-84$.

Tubiello, F.N., C. Rosenzweig, R.A. Goldberg, S. Jagtap, J.W. Jones (2002) Effects of climate change on US crop production, simulation results using two different GCM scenarios. Part I, Wheat, potato, maize, and citrus. Climate Research 20, 2 59-270. 
Turner, D.W. and E. Lahav (1983) The growth of banana plants in relation to temperature. Australian Journal of Plant Physiology 10, 43-53.

Turner, I.M. (1996) Species loss in fragments of tropical rain forest, a review of the evidence. Journal of Applied Ecology 33(2), 200-209.

UNEP (2002) Integrated Assessment of Trade Liberalization and Trade-Related Policies, A Country Study on the Banana Sector in Ecuador. United Nations Environment Program.

U.S. Geological Survey's EROS Data Center (1996) GTOPO30 global digital elevation model (DEM).

Veldkamp, E. A. Weitz, I. Staritsky, and E. Huising (1992) Deforestation trends in the Atlantic zone of Costa Rica, a case study. Land Degradation and Rehabilitation 3, 71-78.

White, M., N. Diffenbaugh, G. Jones, J. Paul and F. Giorgi (2006) Extreme heat reduces and shifts United States premium wine production in the 21st century. PNAS 103(30), $11217-11222$.

Wiley, J. (2008) The banana. Empires, trade wars, and globalization. University of Nebraska Press.

Wittwer, S.H. and N. Castilla (1995) Protected cultivation of horticultural crops worldwide. 1995. Horticulture Technology 5(1), 6-23.

Wunder, S. (2001) Ecuador goes bananas, incremental technological change and forest loss. In Agricultural Technologies and Tropical Deforestation. CAB International. 


\title{
CHAPTER III
}

\section{AN EVALUATION OF UAV SYSTEMS FOR REMOTE SENSING OF BANANA PRODUCTION AND YIELD}

\begin{abstract}
Remote sensing through Unmanned Aerial Vehicles (UAVs) can potentially be used to identify the factors influencing agricultural yield and thereby increase production efficiency. The use of UAVs remains largely untested in tropical agricultural systems. In this study I compared the ease of use and efficiency of multi-rotor and fixed-wing UAV systems equipped with two different sensor systems for mapping spatial patterns of photosynthetic activity in banana plantations in Costa Rica. Spatial patterns derived from photosynthetic indices based on reflected red edge and visible light (ENDVI) and reflected near infrared and red light (NDVI) were then compared to spatial patterns of physical soil quality, irrigation activity, and banana fruit production data. I found that the multirotor UAV system was easier and safer to operate but that the fixed-wing UAV system was much more efficient in areal coverage and extent of imagery acquired per unit time. Spatial patterns of ENDVI and NDVI were significantly positively correlated with several metrics of fruit yield and quality. Irrigating bananas during early stage growth significantly increased both ENDVI and canopy cover. NDVI was not examined for irrigation effects. Spatial patterns of NDVI were not correlated to spatial patterns of physical soil quality. ENDVI was not examined for soil quality effects. These results indicate that UAV systems can be used in banana plantations to help map patterns of fruit
\end{abstract}


yield as well as some of the underlying drivers of yield, thereby helping to increase agricultural efficiency.

\section{Introduction}

A global population projected to reach 9 billion and having increased affluence will drive increased demands for food and agricultural production (Nellemann 2009; Alexandratos and Bruinsma 2012). This demand can be met through expansion of agricultural lands, increases in yields, and/or shifts in dietary habits (Keyzer et al. 2005; Godfray et al. 2010; Machovina and Feeley 2014; Feeley and Machovina 2014). Expansion of agricultural lands is the leading cause of natural habitat destruction (Foley et al. 2005; Foley et al. 2011), and it is predicted that future expansion will require conversion of an additional one billion ha of natural habitats by 2050 , an area approximately the size of the United States (Tilman et al. 2001). The need for future land conversion, however, can be at least partially ameliorated through more efficient use of existing productive lands and increasing yields (Tilman 1999).

Bananas (Musa acuminata) are the developing world's fourth most valuable food crop (Frison et al. 2004) and globally are the 12th most important plant crop by value and quantity. Worldwide, over $100 \mathrm{Mt}$ of bananas are grown annually on an estimated area of approximately 5 million ha (FAOSTAT 2014). Export production, with a volume exceeding $15 \mathrm{Mt}$ and an estimated value of approximately US\$5 billion annually, is concentrated primarily in Latin America, where over $80 \%$ of banana exports originate 
(Evans 2012; FAO 2009; Robinson and Sauco 2010). Costa Rica is the world's second largest exporter of bananas, producing about one-third the quantity of Ecuador that leads the world with annual export production exceeding $5 \mathrm{Mt}$ and US\$2 billion in export value(FAOSTAT 2014).

In many countries, large expanses of tropical forest, especially lowland forest, have been converted to banana plantations (Wiley 2008; Veldkamp et al. 1992), causing widespread decreases in biodiversity and carbon stores. Banana cultivation also requires extensive use of agrochemicals as nutrient sources and biocides, comprising significant portions of the costs of production, and causing downstream environmental effects (Astorga 2005; Marín et al. 2003; Worobetz 2000). Irrigation is increasing in importance because some areas where bananas have been supplied with water solely by rainfall for over a century (e.g., in Costa Rica) are now experiencing prolonged droughts (Portillo 2014). Future projections indicate a drier and hotter climate throughout much of the banana export countries of Latin America, and increased irrigation needs are highly likely throughout the region (Machovina and Feeley 2013).

Better understanding of the relationships between soil factors, water use, and other interacting variables of crop ecology that determine production could potentially lead to increases in yields (Cassman 1999; Mueller et al. 2012), reducing pressure to geographically expand production. An important strategy for improving agricultural productivity and food security is utilizing new technologies to gather information on crop ecology that can help better direct management decisions (Gebbers and Adamchuk 2010; Foley et al. 2011). As a core element of precision agriculture, remote monitoring of crop 
photosynthesis and yields can reveal patterns of stressors affecting crops, enabling managers to adjust treatments to specifically target threatened or affected areas while avoiding treating areas not requiring attention. This could improve yields while reducing input costs and environmental impacts.

Remote sensing platforms with sensors for measuring electromagnetic reflectance patterns from vegetation offer opportunities to identify geographic patterns of crop stressors and can be used to help investigate underlying causes of stress and improve the agricultural management decision making process (Jackson 1986; Plant 2001). Groundbased sensors, as well as sensors mounted on satellites and manned airplanes, have been used to monitor a variety of parameters in managed and natural systems; parameters measured include water stress (Takács and Tamás; Tamás and Lénárt 2006; Jones and Schofield 2008; Jones 1999), pest damage (Prabhakar et al. 2011; Hillnhütter et al. 2012; Nutter Jr et al. 2002), and disease (Zhang et al. 2003; West et al. 2003; Pozdnyakova et al. 2002; Mahlein et al. 2010; Apan et al. 2004), as well as underlying physical variables affecting production, such as leaf area index (Hoffmann and Blomberg 2004; Steltzer and Welker 2006), topography (Florinsky 1998; Hirano, Welch, and Lang 2003), soil quality and nutrient availability (Goel et al. 2003; Apan et al. 2004). Stressors are often visible through remote sensors before the effects can be perceived by the human eye, offering advantages to address problems earlier in their cycle of damage (Jones 2004) and at larger spatial scales. The utilization of spectral reflection patterns of near infrared (NIR) and red light are used via the commonly-applied normalized difference vegetation index (NDVI) (Rouse et al. 1973) to examine spatial patterns of agricultural productivity 
patterns (Leon et al. 2003; Tamás and Lénárt 2006). The NDVI, which indicates the amount of red light absorbed and NIR light reflected, is closely correlated with photosynthetic activity of plants, and spatial patterns of photosynthetic activity can be visualized as varying levels of NDVI. Increased photosynthesis increases crop yields, and spatial patterns of NDVI early in crop development have successfully been used to predict harvest levels many months later (Zarco-Tejada, Ustin, and Whiting 2005; Dobermann and Ping 2004; Leon et al. 2003).

Small Unmanned Aerial Vehicles (UAVs) are rapidly increasing in popularity as a potential tool for monitoring many agricultural practices (Swain, Thomson, and Jayasuriya 2010; Turner, Lucieer, and Watson 2011; Knoth, Prinz, and Loef 2010; Swain, Jayasuriya, and Salokhe 2007; Zhang and Kovacs 2012; Laliberte, Winters, and Rango 2011). UAVs that include multi-rotor, fixed-wing, and lighter-than-air (i.e., balloon or kite) platforms (Inoue, Morinaga, and Tomita 2000) can, in some situations, offer advantages of acquiring aerial imagery at lower costs than manned airplanes or satellites with user-friendly methodology such as easier flight training, rapid field deployment, and quick turnaround of image processing, especially when target areas are small and numbers of images are low. Small, lightweight sensor systems can capture NIR and red light, enabling monitoring of NDVI of vegetation by small, low-cost UAVs. (Tamás and Lénárt 2006; Manera et al. 2010). Their use, however, can be limited by aviation laws, safety concerns, short flight times, weather, or small payload capacity (Hardin and Jensen 2011). 
The goals of this research were to perform initial evaluations of two open-market inexpensive $(<\mathrm{US} \$ 20,000) \mathrm{UAV}$ platforms (one multi-rotor and one fixed wing) for monitoring banana plantations, and to compare remotely-sensed images acquired through two inexpensive sensor systems $(<\mathrm{US} \$ 5,000)$ to recent banana production data. In addition to evaluating ease-of-use of the UAV systems, a goal of the research was to determine how well the remotely-sensed data estimates banana production variables.

\section{Methods}

Two UAV systems were evaluated for remote vegetation sampling potential in commercial banana plantations located near the city of Rio Frio in Heredia, Costa Rica $\left(10^{\circ} 19^{\prime} 30^{\prime \prime} \mathrm{N}, 83^{\circ} 53^{\prime} 11^{\prime \prime} \mathrm{W}\right.$; Fig 3.1a). The study area was located at approximately 100 meters asl on flat topography east of the mountain range that runs north-south through Costa Rica. Between 2008 and 2012, the area received a mean annual rainfall of 4900 $\mathrm{mm}$ (Fig. 3.1b) and had a mean annual temperature of $25^{\circ} \mathrm{C}$. The region was dominated by agricultural activities including banana, pineapple, heart of palm (Bactris gasipaes), and tropical ornamental plant cultivation. The UAV systems were evaluated during the first week of April 2014.

The harvesting methods in these banana plantations provided a unique opportunity to compare remotely-sensed data to banana production data as bananas are harvested from specific areas along numbered cable lines which vary in length from approximately $100-300$ meters that transport bunches to processing facilities, and 
several standard measurements of banana fruit production and quality are recorded for each cable line. Approximately every 9 months, a banana plant produces a single bunch, which is comprised of 5-10 hands which each produce 10-20 bananas (fingers). Typically, the area of harvest encompassed $\sim 50 \mathrm{~m}$ on each side of a cable line. In this study, I compared remotely-sensed data to six banana fruit production measurements: number of boxes produced per ha (one box $=44 \mathrm{~kg}$ ), mean weight of a bunch, mean loss (proportion of bananas discarded from packing due to unacceptable quality), mean number of hands per bunch, mean size of largest banana per bunch, and the mean thickness of a banana on the second hand. Production variables were provided as totals or averages from four-week periods. The mean value per cable line for each variable that was compared to remotely-sensed data was calculated as the mean of the combined values recorded during the 13 four-week sampling periods of 2013 and the first 6 recorded four-week sampling periods of 2014, providing a mean value from 76 weeks of production data.

\section{Multi-rotor System}

Supplied by Elevated Horizons, Inc. (elevatedhorizons.com), the multi-rotor system (Fig. 3.2) was a $66 \mathrm{~cm}$ diameter, $4.4 \mathrm{~kg}$ hexacopter powered by a single 11000mah, 14.4v, 4-cell LiPO battery. Flight control was managed with a flight controller (DJI Wookong; dji.com) integrated with an iPad Mini (Apple; apple.com), relayed via a backpack-mounted antenna system. Flight plans were made by touching desired waypoints on satellite images of the study area downloaded from Google Maps 
on the iPad. Takeoff and landings were performed via manual control, but image-capture flight patterns were done under automated control by the flight control software.

The UAV was outfitted with a 231 gram Canon SX260 camera modified by LDP, LLC (maxmax.com) into a "vegetation stress camera" by converting the red channel to capture light in the red-edge portion of the spectrum. The system captures images in the following FWHM (Full Width at Half Height Measurement) wavelengths in the red, blue, and green channels: Red Edge: $688-739$ nm; Blue: $408-510 \mathrm{~nm}$; Green: $481-535 \mathrm{~nm}$. It acquires 12.1 megapixel spatial resolution images with 8-bit radiometric resolution and is equipped with a 5.7-18.8 $\mathrm{mm}$ zoom lens. Focal length for all flights was set at $5.7 \mathrm{~mm}$ and formatting was set to $4: 3$ large ( $4000 \times 3000$ pixels). The camera is equipped with an internal GPS which was selected to stamp each image file with geographic coordinates. Images were stored on $16 \mathrm{~GB}$ removable storage cards.

Five flights were performed to cover a total of 20 cable lines; each flight was vertically launched and landed from roads adjacent to targeted banana fields. Sixteen of the cable lines (cables labeled from 15 to 44 ) were located within a single $\sim 1.3 \mathrm{~km} 2$ area and four (cables labeled 123-126) were located within a $0.32 \mathrm{~km} 2$ area located approximately $3 \mathrm{~km}$ from the first area. Flights were made between 10:00AM and 2:00PM local time on April 3, 2014. Flights were programed to complete a route that would fly a back-and-forth "lawnmower" pattern traveling $5 \mathrm{~m} / \mathrm{s}$ at $80 \mathrm{~m}$ altitude directly above and parallel to banana harvesting cable lines, covering $400 \mathrm{~m}$ distance along four cable lines or approximately $12,000 \mathrm{~m} 2$ per flight. The camera was set to capture photos at 7 second intervals. Each flight lasted approximately 16-18 minutes (depending on 
winds), flew a linear distance of $1.5 \mathrm{~km}$, and recorded $\sim 16$ ha of imagery at $2.4 \mathrm{~cm}$ resolution. Images were recorded with approximately 50\% forelap and no sidelap.

Post-flight images were sorted to remove takeoff/landing images lower than $80 \mathrm{~m}$ altitude, blurry images, images not located along cable lines, and images of cable lines where banana production was recently abandoned. Images were then sorted on the basis of their corresponding cable lines. From the image sets covering areas harvested along each cable line, 20 locations were selected via a stratified random sampling by dividing each side of a cable into 10 approximately-equal-sized zones and randomly selecting the approximate center of one of the four quadrants in each zone. At each sampling location, the closest $1002 \times 1002$ pixel $(627.5 \mathrm{~m} 2)$ that covered only bananas (no roads, paths, canals, or other vegetation types) was selected and cropped from the photo using ImageJ (Rasband 2014). Cropped images were then processed to (1) calculate ENDVI (enhanced normalized difference vegetation index) images of the samples and (2) estimate density and dispersion patterns of individual banana plants.

ENDVI was calculated using an ImageJ macro developed and supplied by LDP, LLC using the following equation:

\section{$E N D V I=\left((\right.$ NIR + Green $)-\left(2^{*}\right.$ Blue $\left.)\right)$ \\ $((\mathrm{NIR}+$ Green $)+(2 *$ Blue $))$}

In the above equation NIR is the Red Edge band. This is different from most standard definitions of NIR, which are $\sim 750-1000 \mathrm{~nm}$ or up to $1300 \mathrm{~nm}$, and instead uses the red edge, where sharp changes in leaf reflectance occur between 680 and $750 \mathrm{~nm}$. As canopy cover is reduced or chlorophyll pigmentation is reduced by stress of many factors, 
the camera will record less reflected visible green light and more red edge and blue light. This formula sums the NIR and green channels together for the reflective channel. The blue channel is multiplied by two to compensate for the NIR and green channels being summed together (LDP 2014). Raw ENDVI values were scaled (highest raw ENDVI set to equal 1.0 and lowest raw value set to -1.0 ) and used for further analysis. The mean scaled ENDVI value of the 20 random samples from each cable line was compared to the seven banana production variables recorded for corresponding cable lines.

Post-flight sample images were also processed to estimate plant density and dispersion patterns by visually identifying individual plants and marking their location using the Point Picker plugin for ImageJ, and exporting text files for each image that indicated total number of plants per sample area and x,y pixel coordinates of their locations. Plant locations were analyzed for clumping with a nearest neighbor distance spatial index (R splancs; http://cran.r-project.org/web/packages/splancs/index.html). This index calculates the mean nearest neighbor distance (NND) for each plant in each plot. The plants within the plot are then randomized 1000 times without replacement and the nearest neighbor distance is calculated during each permutation. The spatial index within each plot is calculated by:

Spatial Index $(\mathrm{SI})=($ observed mean NND - median randomized NND) / standard deviation of randomized NND

SI decreases with clumping of individuals. If the SI value is less than -2 then the species is significantly clumped. Between -2 and 2 indicates no significant difference from random distributions, while SI values greater than 2 demonstrate a regular 
distribution. Plot level SI values were calculated as the mean of the SI values within the plot. The average distance between plants (x,y coordinate locations of pixels at the center of a plant) was calculated using R (R Development Core Team 2014). Average density, clumping, and distance between plants for each cable line were compared via regression analysis using Microsoft Excel (2010) to the recent historical banana production data from that line.

An additional flight was performed over an area of bananas planted with seedlings in January 2014 to compare the effects of supplying irrigation during lower rainfall periods on banana productivity over the first 13 weeks of plant growth in the field. Bananas reach maturity and fruit harvest in approximately 9 months. Areas receiving rainfall and irrigation were compared to areas supplied with only rainfall. The flight was performed at $30 \mathrm{~m}$ altitude, providing $1.2 \mathrm{~cm}$ ground resolution. Twenty $5 \times 5 \mathrm{~m}$ samples were randomly selected from the flight imagery from each of the irrigated and nonirrigated areas. Sample imagery was processed for ENDVI as described above and was analyzed in ImageJ to calculate canopy cover by converting post-flight images to binary images (vegetation set to black \& bare ground set to white), then creating histograms of the images and recording numbers of black and white pixels. This enabled calculation of the area of canopy cover in each $25 \mathrm{~m} 2$ sample area and average area of canopy cover of samples in irrigated and non-irrigated areas.

\section{Fixed-Wing System}

Supplied by MarcusUAV, Inc. (marcusuav.com), the fixed wing UAV system (Fig 3.3) was a $2.5 \mathrm{~kg}$ delta-wing design with a $175 \mathrm{~cm}$ wingspan, powered by two 2700 
$\mathrm{mAh}, 14.7 \mathrm{v}$, 4-cell LiPO batteries. Manual flight control during takeoff and landing was performed with a Spektrum DX8 RC controller. Mission planning and automated flight control was performed using Mission Planner 1.22.99 on a laptop computer, relayed via a ground-based radio-modem antenna. A small video camera mounted in the nose of the UAV relayed live video footage of the flight path to a ground-based tracking antenna. All automated flight operations and video processing were managed via a single laptop computer connected to the antenna system. Flight plans were made creating survey grids using the Auto Waypoint and Polygon tools in Mission Planner on imagery downloaded from Ovi Satellite Maps, which provided better high-resolution coverage of the region than Google Maps. Takeoff and landings were performed via manual control, but imagecapture flight patterns were under automated control by the flight control software.

The fixed-wing UAV was outfitted with a 90 gram Tetracam ADC Micro (tetracam.com), which was mounted on a motorized roll stabilizer. The Tetracam Micro captures Near Infrared, Red, and Green wavelengths similar to Landsat Thematic Mapper bands TM2, TM3 and TM4. Wavelengths recorded are Infrared: 760-900 nm (recorded on red channel), Red: 630-690 nm (recorded on green channel), and Green: 520-600 um (recorded on blue channel). The system has a 3.2 megapixel resolution (2048 x 1536) sensor and a fixed $8.43 \mathrm{~mm}$ lens. Images were stored on $16 \mathrm{~GB}$ removable storage cards. Geographic locations of camera trigger points were recorded by the Tetracam from the UAV's flight controller GPS.

Flights were made over a different geographic location and cable lines than those for the fixed-wing system. Prior to the flights, images of a white Teflon calibration plate 
were recorded with the Tetracam under ambient light conditions. The UAV was launched from a dual slide-rail launcher constructed from PVC piping and powered by a $15 \mathrm{~m}$, triple-cord bungee line staked into the ground approximately $30 \mathrm{~m}$ in front of the UAV. A foot operated trigger released the UAV. Launches were performed from an athletic field located within $0.5-2 \mathrm{~km}$ of the origins of the onset of imagery capture. Landings occurred at the same location as launches, and were achieved via manual triggering of a parachute deployment or by manually slide landing the UAV on the grassy field. Three flights were performed, reaching $260 \mathrm{~m}$ altitude image capture elevation, traveling at $16 \mathrm{~m} / \mathrm{s}$, lasting from 20-22 minutes, flying linear distances of $11.7 \mathrm{~km}, 16.4 \mathrm{~km}, 16.5 \mathrm{~km}$ and recording imagery covering 165 ha, 186 ha, 164 ha respectively. Images were recorded with approximately $60 \%$ forelap and $40 \%$ sidelap and a pixel resolution of $10 \mathrm{~cm}$.

Post-flight images were transferred to a laptop and visually sorted to remove takeoff/landing images lower than $260 \mathrm{~m}$ altitude and blurry images. Images were processed into false-color infrared images and NDVI classified images using the Teflon standard images and Pixel Wrench, the image processing software supplied by Tetracam. Using Agisoft Photoscan Professional, I attempted to mosaic and orthorectify images from each of the flights, but only the second flight provided sufficient image quality and overlap to enable the creation of a quality mosaicked single image using automated methods of the software. All banana production data comparisons were performed on data extracted from the mosaic from this flight.

The orthorectified mosaic of the flight was imported into ArcGIS. A vector map indicating locations of cable lines, supplied by growers, was also imported. A total of 23 
cable lines with active banana production areas were identified. Along each of these cable lines, 20 locations were identified visually for sampling NDVI values from the NDVI mosaic. NDVI was calculated as (NIR-R)/(NIR+R). Sample locations, consisting of a 10 $\mathrm{m}$ diameter $(78.5 \mathrm{~m} 2)$ circular area, were sampled via a stratified random sampling method (similar to the multi-rotor image processing methods described above) along both sides of a cable line, selecting locations that did not include roads, canals, or unplanted areas. The mean NDVI value for a cable line was calculated as the combined mean of pixels in all 20 sample location areas along each cable line. A vector map indicating locations of samples for determining soil classifications, supplied by growers, was also imported. These classifications were made based on soil core samples previously made by growers at the specific locations. Soils at sample sites were classified on a four-tier scale (I-IV) of most to least favorable classes, respectively, for banana cultivation based on physical soil characteristics including texture, structure, portion of coarse fragments, consistence, and drainage. At each core sample location, a $10 \mathrm{~m}$ diameter $(78.5 \mathrm{~m} 2)$ circular area was selected from the NDVI mosaic. Only soil sample locations where the $10 \mathrm{~m}$ diameter NDVI sample included bananas alone (no other vegetation types) were included in analysis. The mean NDVI value for each soil classification was determined by calculating the mean of all pixels from all soil sample locations for each soil classification level. 


\section{Results}

\section{Multi-rotor System}

The five flights produced 1140 images. Depletion of the camera battery on one flight resulted in insufficient area covered for two cable lines. Sorting resulted in 473 raw images covering 16 cable lines that could be to be used for further processing. A total of $32024 \times 24$ m sample images (20 for each of 16 cable lines) were extracted and processed into ENDVI images (Fig 3.4.), and average ENDVI pixel value per image was calculated for each cable line. When sorted by mean ENDVI values, the resulting ENDVI sample images revealed a visible difference among cable lines (Fig. 3.5). Mean ENDVI values varied five-fold from lowest to highest values among cable lines $(0.12-0.61$; Fig. 3.6). Mean ENDVI per cable line was positively correlated with 3 production variables (Fig. 3.7a-c): mean bunch weight, mean hands per bunch, and mean thickness of a banana on the second hand, but was not significantly correlated with the other three production variables. There was a positive, but non-significant, trend of greater mean boxes per hectare in areas with higher ENDVI (Fig. 3.7d). When examining relationships among banana production variables, the mean weight of a bunch is strongly correlated with mean boxes per hectare (Fig. 3.7e).

A significant negative correlation existed between density of plants and mean hands per bunch (Fig. 3.7f), but no significant relationship was found between density of plants and any other production variables. Increased mean distance between plants was significantly positively correlated with mean bunch weight (Fig. 7g) and mean hands per bunch (Fig. 3.7h). A strong positive correlation existed between ENVDI and increased 
mean distance between plants (Fig. 3.7i). No significant relationship occurred between clumping of plants and any of the production variables.

A significant difference in plant canopy cover was found between irrigated and non-irrigated 13 -week-old bananas plants (t-test $\mathrm{p}<0.05$ ) with the irrigated areas having an average of 1.2 times greater cover. ENDVI was found to be significantly different between the two treatments (t-test $\mathrm{p}<0.05)$ with irrigated areas having ENDIV values that were 2.3 times higher than non-irrigated areas (Fig. 3.8). No significant correlation exists between Mean ENDVI and Canopy Cover when irrigated ( $\mathrm{r} 2=0.0025 ; \mathrm{p}=0.83)$ and non-irrigated treatments $(\mathrm{r} 2=0.048 ; \mathrm{p}=0.35)$ are analyzed separately.

\section{Fixed-Wing System}

Sorting produced 269, 259, and 294 images from flights one, two, and three, respectively. Attempts to mosaic all images from the first and second flight were not successful. Flight two produced better results for mosaicking, but required several rounds of utilizing manual tie points to correctly match and align adjacent images and groups of images. The mosaic from the second flight contained some areas with slight misalignment among adjacent images, but provided sufficient accuracy to locate sample points along cable lines and soil sample locations (Fig. 3.9a).

Mean NDVI values from the 23 cable lines ranged from 0.20 to 0.35 with a mean value across all cable lines of 0.26 . In general, the region north of the road bisecting the mosaic image exhibited higher NDVI values (Fig. 3.9b). Mean NDVI was significantly 
positively correlated with four banana fruit production variables: mean bunch weight (Fig. 3.10a); mean number of hands per bunch (Fig. 3.10b); mean length of largest finger (Fig. 3.10d); mean boxes per hectare (Fig. 3.10e). Mean NDVI was significantly negatively correlated with mean loss (Fig. 3.10f). No significant relationship existed between mean NDVI and mean banana thickness on the second hand (Fig. 3.10c).

A total of 49 soil sample locations (12 from Class I, 12 from Class II, 13 from Class III, 12 from class IV) were located in areas containing only banana plants in the mosaic. NDVI values from the soil classes ranged from 0.23 to 0.27 . No significant difference in average NDVI value existed among the soil classes.

\section{Discussion}

\section{Comparison of UAV Systems}

The two UAV systems offered contrasting advantages and capabilities. The primary differences were related to (1) ease of use and (2) flight distances. The multirotor system offered a higher degree of simplicity in overall operations. It was easier to launch and land, which was achievable vertically in an open area as small as $3 \times 3 \mathrm{~m}$ immediately adjacent to subject banana fields. Transport of the equipment and setup for launches was simpler and quicker. Launches and landings occurred at low speeds under a higher-degree of manual control and less opportunity for pilot error or crashes.

In comparison, the fixed-wing system required more time to setup and launch. It also required locating large, flat grassy fields for safe launches and landings. Manual flight control of the fixed wing system requires more skill and training than the rotary 
wing system. During manual landings that involved skidding the fixed-wing UAV on the grassy field, the rapid speed of the UAV was challenging and rough landings sometimes occurred, especially under windy conditions. Over time this could lead to gradual damage and increased risk of an accident. Deployment of the parachute for landing was preferred but required precise timing and altitude in order to achieve landings within the confines of an athletic field, especially if surrounded by large trees. It was apparent that, for new users, learning to fly a multi-rotor system would be much easier, and the likelihood for crashes less. For rapid deployment and ease of use, the multi-rotor system is preferable.

The ease-of-use advantage of the multi-rotor system, however, was overshadowed by the much greater level of image capture per unit time of the fixed-wing system and the much longer distance and area of coverage achievable with the fixed-wing system. The fixed-wing system traveled $3 \mathrm{X}$ faster and in a single flight, that was limited by battery capabilities, covered 10X more linear distance ( $>16 \mathrm{~km}$ vs. $1.5 \mathrm{~km})$, acquiring more images per unit time and energy. Although the fixed-wing system was flown at a higher altitude (260 $\mathrm{m}$ vs $80 \mathrm{~m}$ ) resulting in lower spatial resolution (larger pixel sizes)(10 cm vs $2.5 \mathrm{~cm}$ ), it could be flown at a lower altitude to record higher spatial resolution images.

The two camera systems also offered contrasting capabilities and advantages. The converted Canon camera had a larger sensor size than the Tetracam Micro (4000x3000 vs. $2048 \times 1536$ pixels), which enabled higher spatial resolution images to be recorded per flight line when flown at an equal altitude. Therefore, this required fewer flight lines and less total flight distances to record an equal area at an equal spatial resolution, and would also require fewer flights to record larger regions. The GPS signal on the converted 
Canon camera, however, was inaccurate and imprecise and rendered the creation of mosaics from imagery challenging. Utilizing the more accurate flight-control GPS for camera triggering and recording of imagery location instead of timed triggering would be a great improvement of the Canon-based system. The imagery captured from the Canon was also able to be opened and manipulated directly by any image processing software, whereas the imagery acquired on a Tetracam required the use of Pixel Wrench for processing. Although it may have made a negligible improvement in the current study, if comparisons of imagery from the Canon are to be made across different ambient light conditions (season, time of day, weather), utilizing a calibration standard would be necessary.

Improvements in the ability to mosaic imagery from banana plantations could be made by increasing the forelap and sidelap. Levels of approximately $60 \%$ forelap and $40 \%$ sidelap were not sufficient, perhaps due to the largely featureless and monotonous nature of large commercial banana plantations. A minimum of $80 \%$ forelap and $60 \%$ sidelap would be recommended for future flights. Recording and including flight attitude data in the input parameters used in mosaicking software would also improve alignment. Adding pitch stabilization to the UAV might improve image quality.

\section{Imagery Patterns and Banana Productivity}

Results indicated that both ENDVI calculated from the Canon camera data and NDVI from the Tetracam data reveal patterns in plant productivity and are positively correlated with banana fruit production values. Both indices incorporated reflected radiation from banana plants and soils between plants and both are influenced by plant 
productivity as well as density and canopy cover. No other published studies report ENDVI values from banana plantations. NDVI values used to identify banana plantations have been reported to range from mean values of 0.1 to 0.573 (Johansen et al. 2009), and the results of this study $(0.2-0.35)$ are within this range. Areas along cable lines exhibited much larger differences in reflectance indices for ENDVI, with a 5-fold difference across the 16 cable lines, than NDVI, which varied by $43 \%$ across the 23 cable lines.

The NDVI values were significantly correlated with more banana production variables than ENDVI values (5 vs. 3 variables), but this may be the result of sample size (23 cable lines sampled for NDVI vs. 16 cable lines sampled for ENDVI). Both ENDVI and NDVI were significantly correlated with mean bunch weight and mean hands per bunch. Only ENDVI was significantly correlated with mean thickness of a banana on the second hand, and only NDVI was significantly correlated with mean length of largest finger, mean boxes per hectare, and mean loss.

The strong positive correlation between ENVDI and mean distance between plants may indicate that competition between plants for resources (light, water, and nutrients) may affect plant productivity. It would be expected that with increased distance between plants, ENDVI would be lower as soils would be more exposed between plants. However, the upper limits of distances among plants that exist within plantations may result in lower competition and therefore higher productivity and canopy cover without increasing soil exposure. This may also be reflected in the significant positive trend that mean bunch weight and mean hands per bunch both exhibit with increased distance 
between plants. My results indicate that as density of plants decreases, mean hands per bunch increases. One underlying possibility for variation in plant distance may be that in older plantations, banana plants tend to "wander" off their original straight planting lines as the most promising suckers are selected from mother plants for the next generation of fruit production. Although selection is supposed to occur along the parallel lines of original plantings, variation does occur and can lead to clumping of plants and potential increased levels of competition. However, no significant relationships were found between clumping and banana fruit production variables.

The examination of the effects of irrigation on ENDVI levels of early-stage bananas during a drier period of the year revealed the value of irrigation to plant growth during drier periods. The lack of a significant correlation between ENDVI and Canopy Cover when irrigated and non-irrigated treatments were analyzed separately indicated that the vegetation indices may be more sensitive to changes in banana photosynthetic activity than canopy cover. On average from 2010 to 2013, the first 13 weeks of the year experienced approximately $23 \%$ less weekly rainfall than the remainder of the year. Irrigation is likely to become more important in commercial banana export regions of Latin America as regional climates are projected to become hotter and drier (Machovina and Feeley 2013). In recent years, Costa Rica has experienced pronounced droughts during the first few months of the year (Portillo 2014). Starting approximately two years ago, irrigation systems are increasingly being installed in banana plantations throughout the Caribbean growing region of Costa Rica as droughts have recently become increasingly common. Irrigation has not been necessary in the area since the first 
plantations were established there in the late 1800s. The increased ENDVI in irrigated lands and the relationships of ENDVI with banana fruit production variables indicate that irrigation could improve banana yields during dry periods. Increased irrigation may be required to maintain sufficient fruit production for commercial growing operations as regional climate changes. UAVs may be a valuable tool for monitoring irrigation needs.

The comparison of NDVI values to soil quality did not reveal any relationships. This may be due to the sampling of $10 \mathrm{~m} 2$ areas around the soil analysis points and the potential for finer spatial variation in soil quality than captured in this sampling area for mean NDVI values. Finer-scale variation of soil quality may be more difficult to detect. Also, it is possible that the physical variation in soil quality may not have strong effects on banana plant productivity and therefore NDVI or the boundaries defining the different soil classes are not accurate.

This study indicates that both ENDVI and NDVI indices are valuable for estimating spatial patterns of banana fruit productivity. Future recommended research would involve mapping larger areas of banana production with both camera platforms and comparing the same geographic area with both platforms to reveal any technical advantages of either platform. As an economic comparison, the Tetracam costs approximately $5 \mathrm{X}$ more ( $<$ US $\$ 5,000$ vs. $<$ US $\$ 1,000$ ). Even given the limitations for learning to fly a fixed-wing UAV and the increased risk for crashes, the drastically increased rate of area coverage of the fixed wing system warrants its preferential use in large agricultural settings. Further improvements in automation (take-off and landing) 
that limit manual flight as well as failsafe parachute deployment during instances of loss of flight control would greatly expand the system's utility and safety.

The results of this study show that small UAVs equipped with camera sensor systems can be used to successfully map spatial variation in NIR and visual light reflectance patterns that are correlated with banana fruit production measures. It is therefore possible to map much larger areas of banana cultivation and classify areas where fruit yield and fruit sizes are likely higher or lower. This can enable managers to see geographic regions of their plantations where yields can potentially be increased by addressing stressors in areas with lower NDVI or ENDVI. Geographic patterns of these indices are of a much finer-scale than the averages of fruit production attained through measures taken directly on fruit combined from a single cable line, and potentially enable addressing multiple target locations within a single cable line. Further investigation of the underlying variables affecting spatial patterns of ENDVI and NDVI in banana plantations by UAVs is warranted, including comparisons of geographic patterns of the indices against topography, drainage, nutrient availability, disease, pests, and more thorough investigation of soil types. Utilizing UAVs to detect patterns of productivity and underlying causes of variation may enable management scenarios that can address problems and improve yields. Improving yields will in turn allow for greater efficiency, decreasing environmental impacts of banana cultivation as global demand for food increases. 


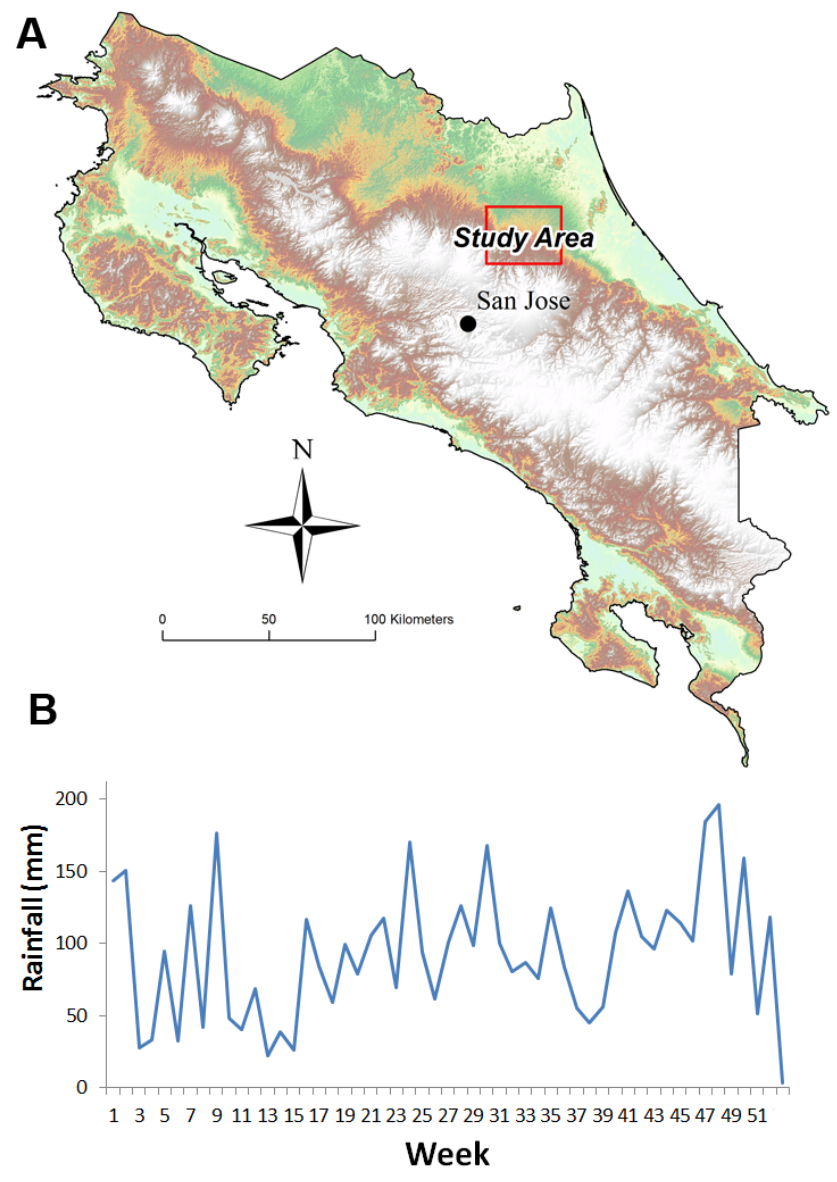

Figure 3.1. (a) An elevation map indicating study area in relation to San Jose, the capital of Costa Rica, and (b) weekly mean rainfall beginning Jan. 1 (2008-2012) at the study area. 

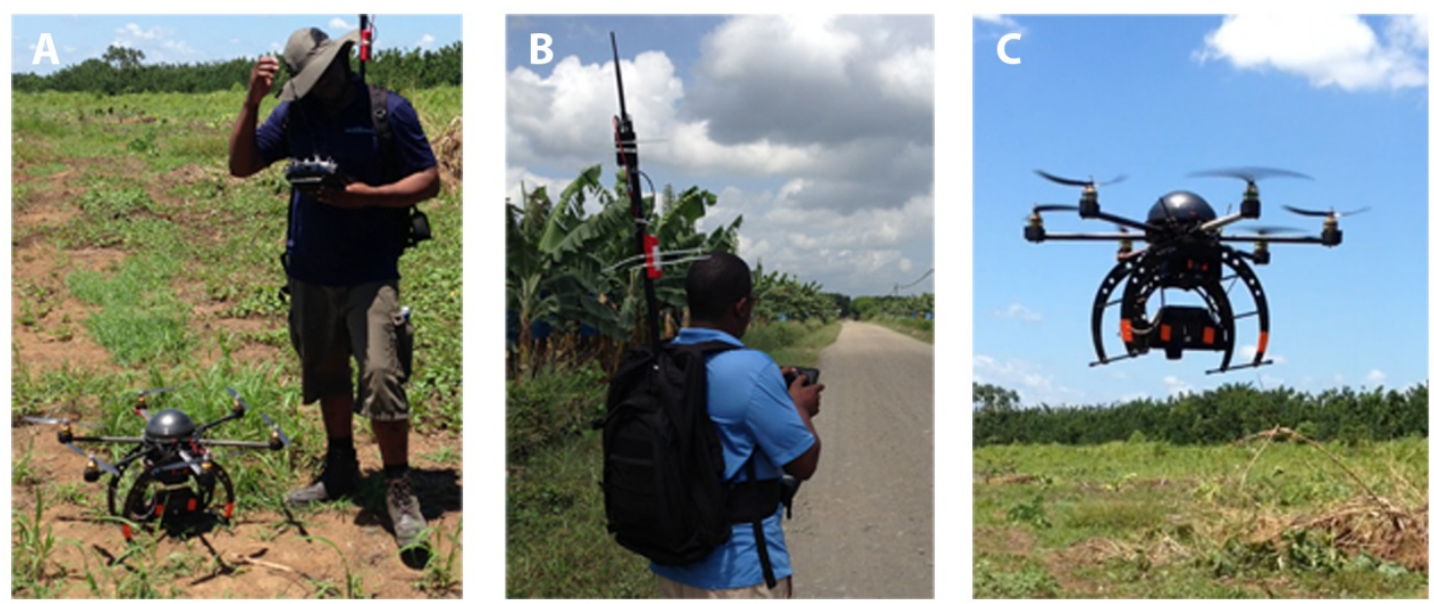

Figure 3.2. Rotary Wing UAV showing (a) approximate size and RC controller, (b) backpack antenna system for location tracking and flight control, and (c) UAV during launch. 

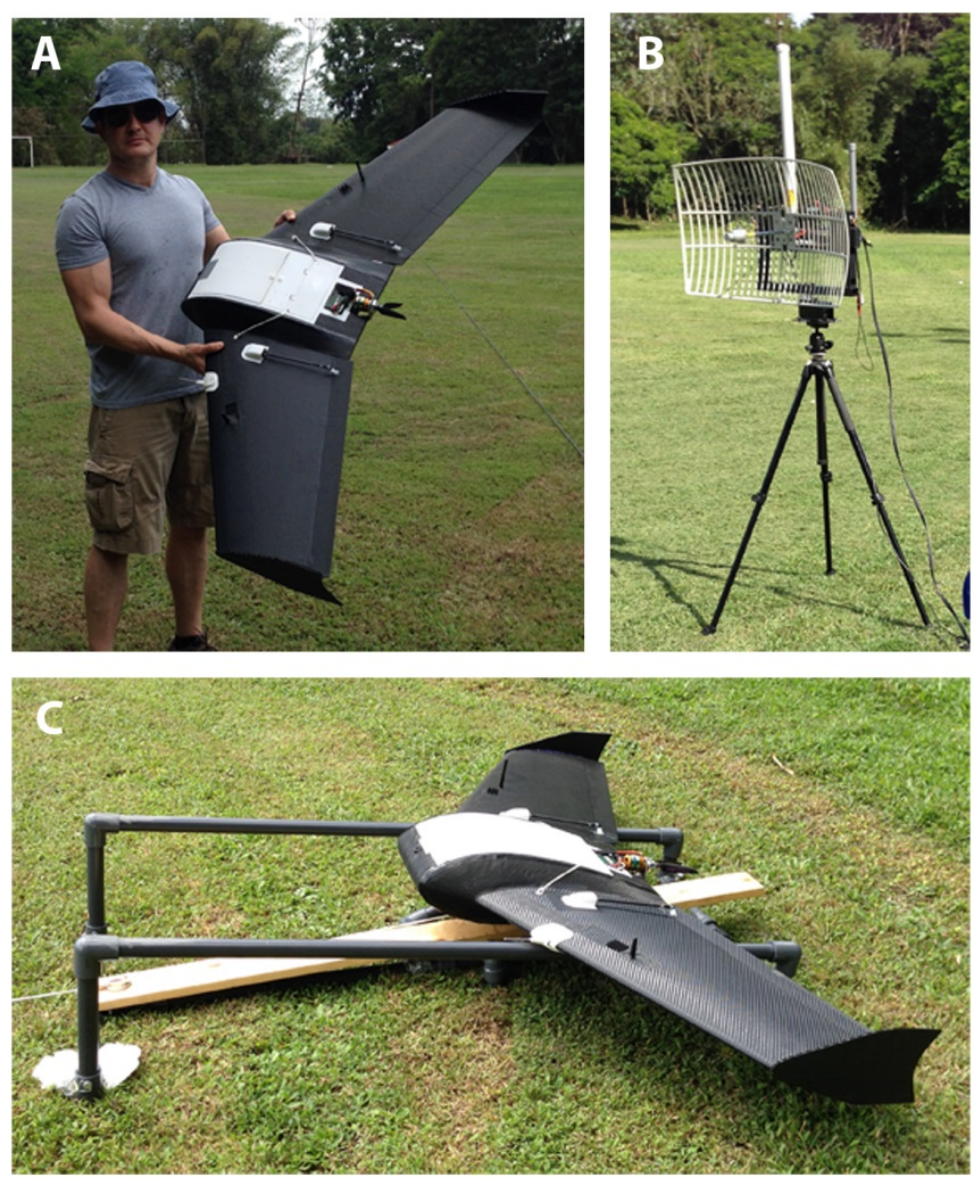

Figure 3.3. Fixed Wing UAV showing (a) approximate size, (b) antenna system for location tracking and live video capture, and (c) UAV mounted on launcher. 

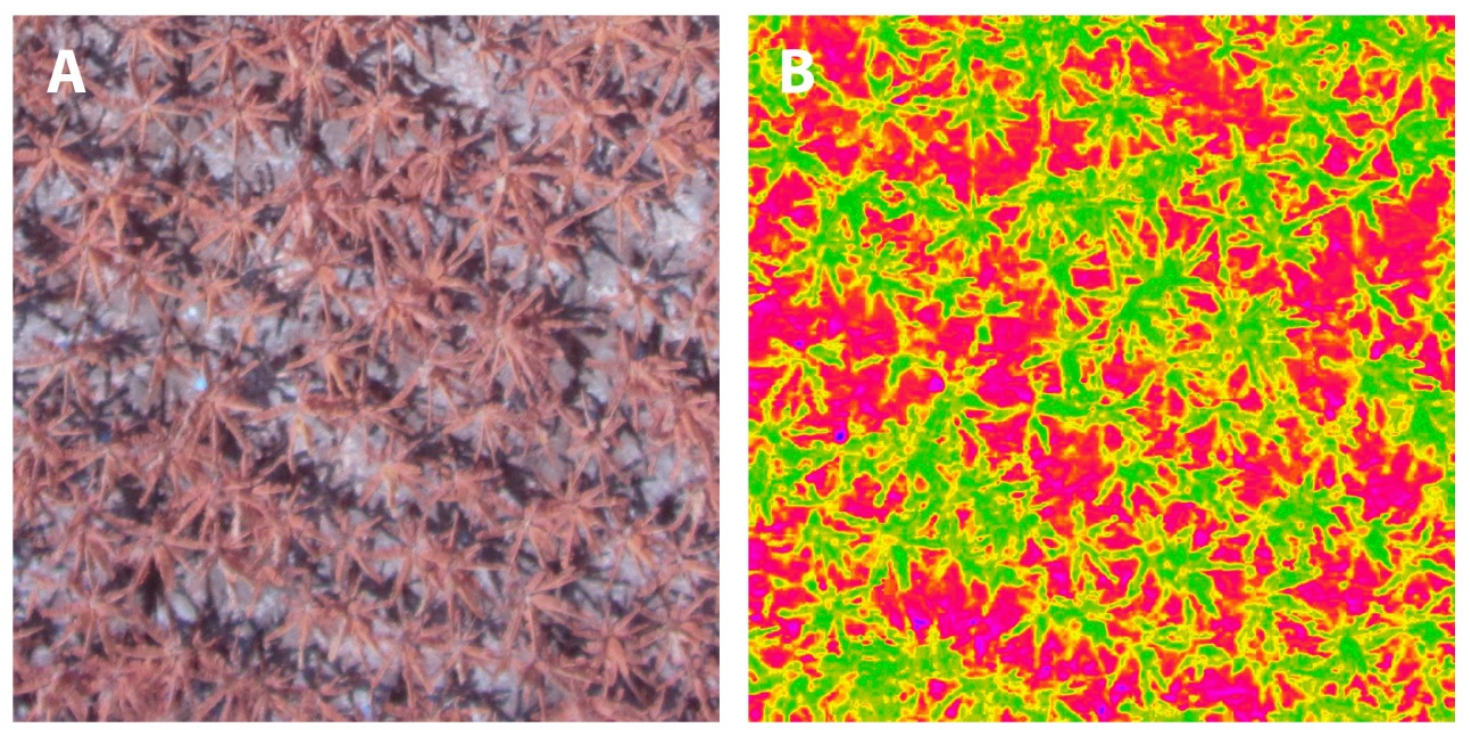

Fig 3.4. Example of (a) $627.5 \mathrm{~m}^{2}$ sample plot false-color image cropped from a raw image from the modified Canon SX260 camera and (b) its conversion into a scaled ENDVI image. 


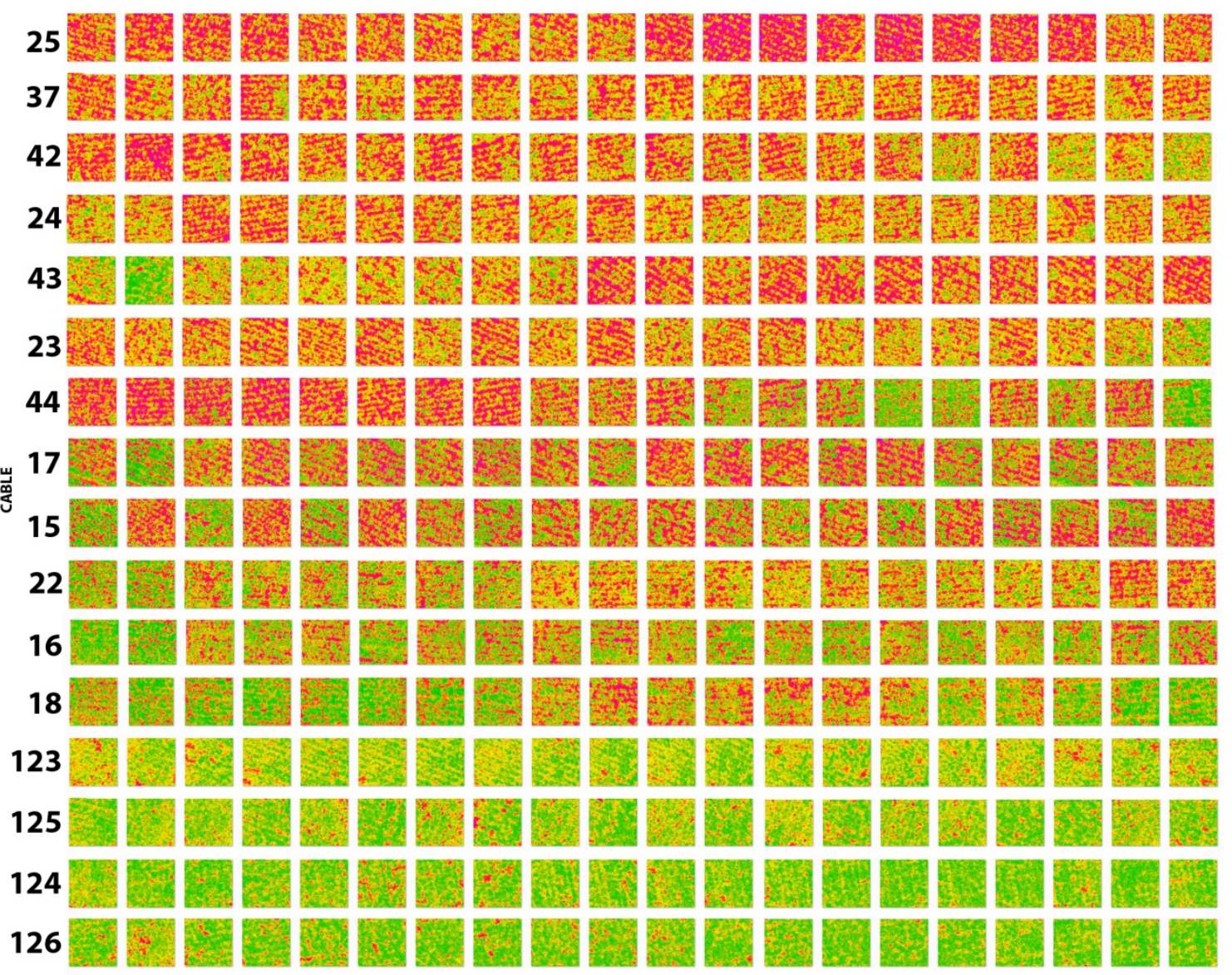

Figure 3.5. ENDVI processed images of 20 random samples selected from harvest areas that supply cable lines. Green = higher ENDVI values, yellow = moderate ENDVI values, red = lower ENDVI values. Cable lines are ranked from lowest to highest mean ENDVI. Cables 15-44 are geographically located within close proximity (a single $\sim 1.3 \mathrm{~km}^{2}$ area), while cables $123-126$ are located approximately $3 \mathrm{~km}$ away. 




Figure 3.6. Mean ENDVI value ranked by cable line. 
A

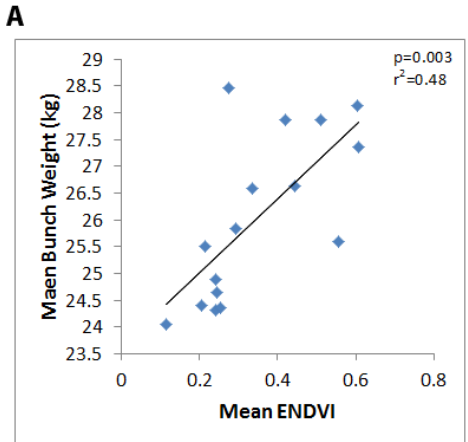

D

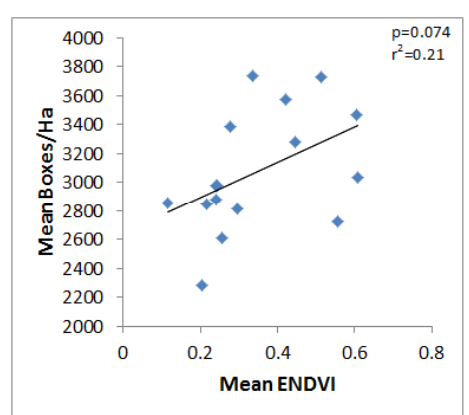

G

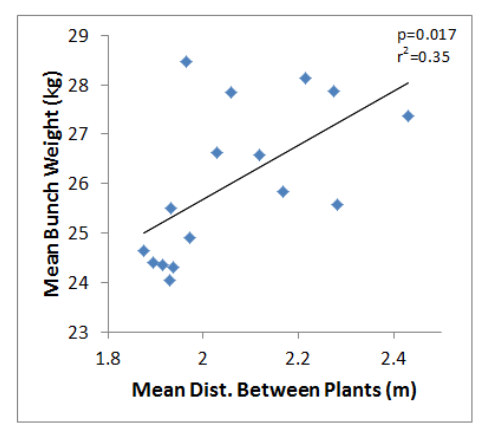

B

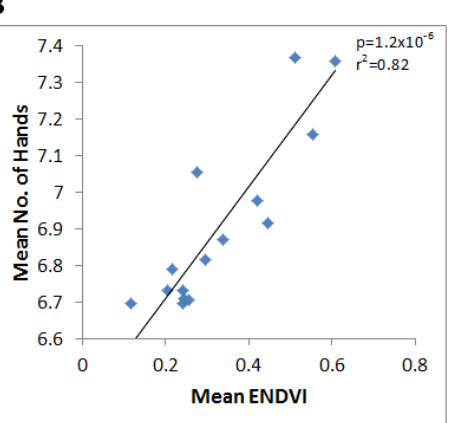

E

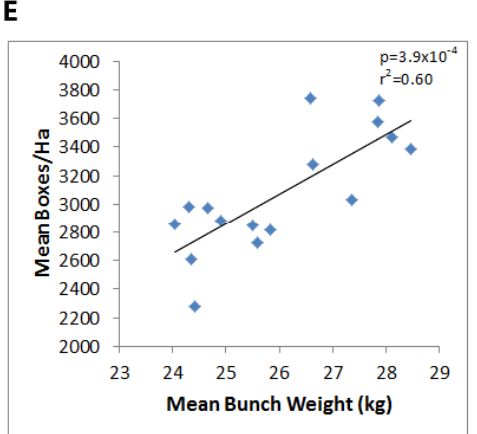

H

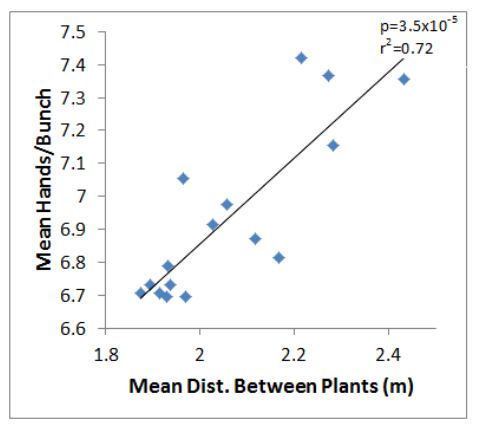

C

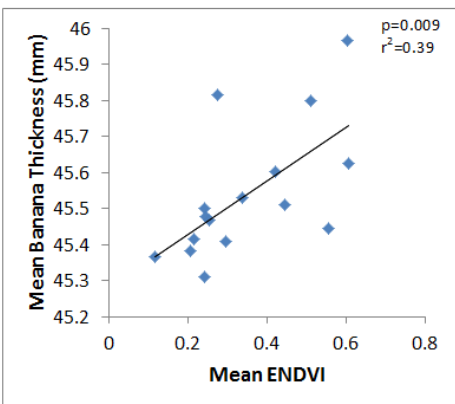

$\mathbf{F}$

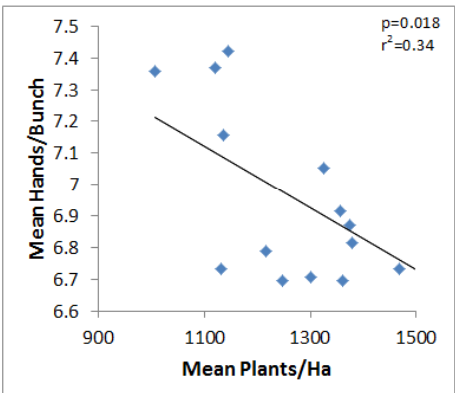

I

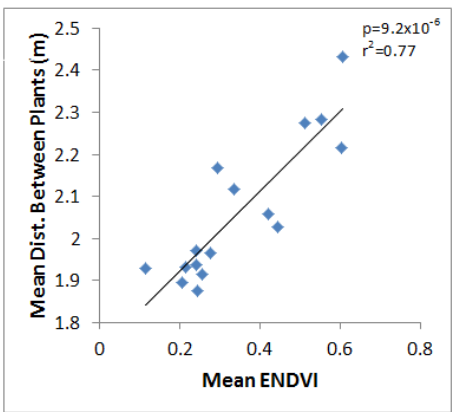

Fig. 3.7. Relationships among variables remotely sensed with modified Canon SX260 camera and banana production measures: (a) Mean ENDVI and Mean Bunch Weight; (b) Mean ENDVI and Mean Number of Hands per Bunch; (c) Mean ENDVI and Mean Banana Thickness on Second Hand; (d) Mean ENDVI and Mean Boxes Per Hectare; (e) Mean Bunch Weight and Mean Boxes Per Hectare; (f) Mean Plant Density and Mean Number of Hands per Bunch; (g) Mean Distance Between Plants and Mean Bunch Weight; (h) Mean Distance Between Plants and Mean Number of Hands Per Bunch; (i) Mean ENDVI and Mean Distance Between Plants. 


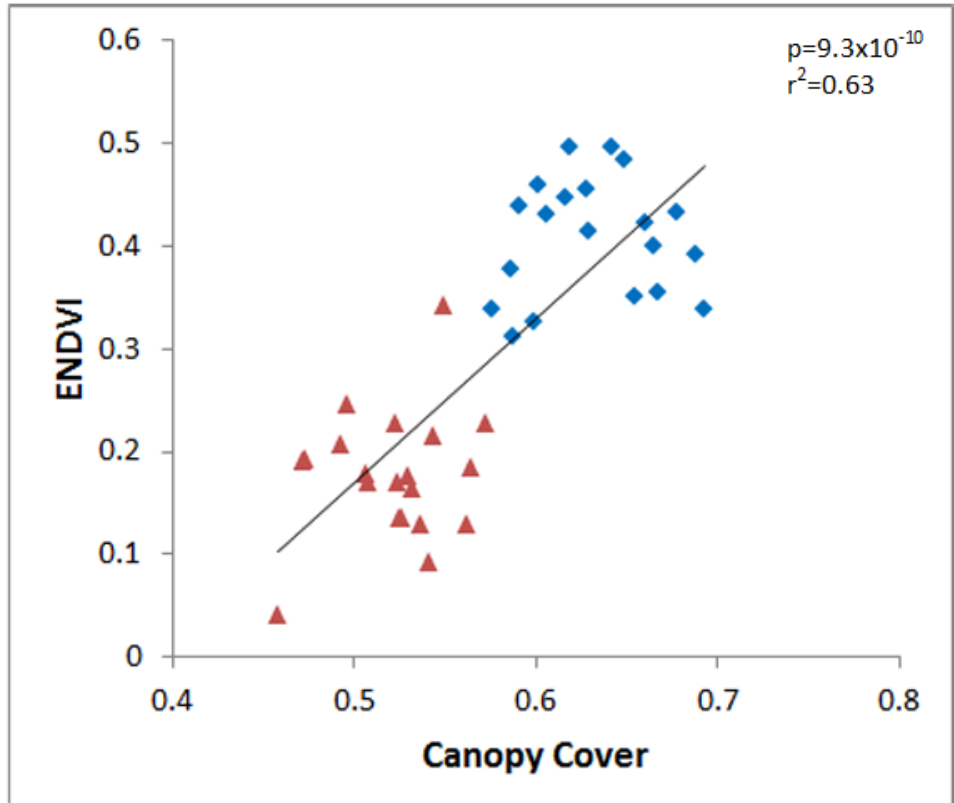

Fig. 3.8. Relationship between Mean ENDVI and Canopy Cover. Blue diamonds are sample plots from irrigated fields, red triangles are from non-irrigated. 

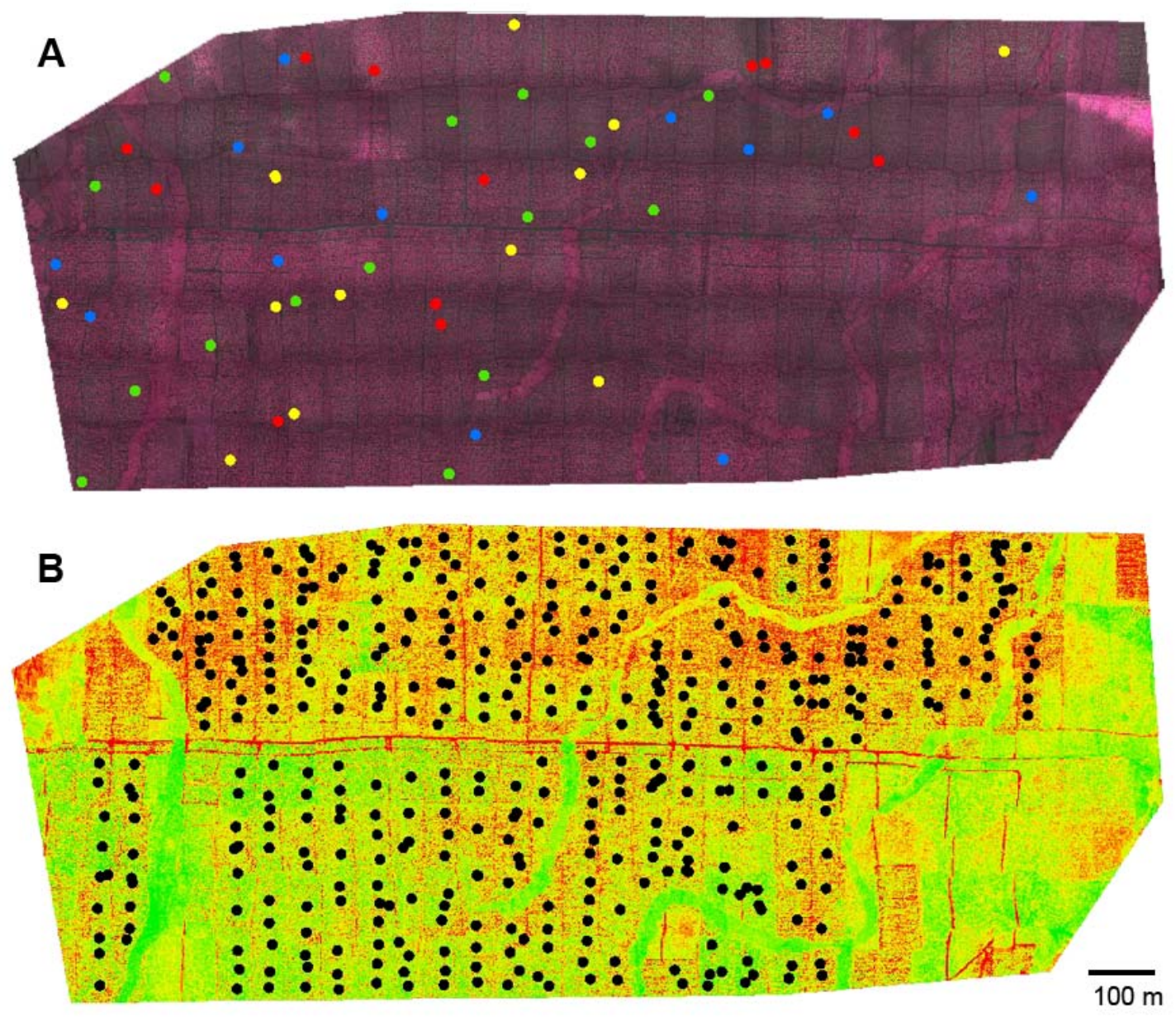

Fig. 3.9. (a) Locations of soil samples on mosaic of false-color imagery acquired with Tetracam Micro. Green = soil class I $(\mathrm{N}=14$, blue $=$ soil class II $(\mathrm{N}=12)$, yellow $=$ soil class III ( $\mathrm{N}=12)$, red = soil class IV $(\mathrm{N}=12)$. (b) Locations of samples of NDVI values taken along cable lines $(\mathrm{N}=460)$. Green $=$ high NDVI levels, yellow = moderate NDVI levels, red $=$ low NDVI values. 
A

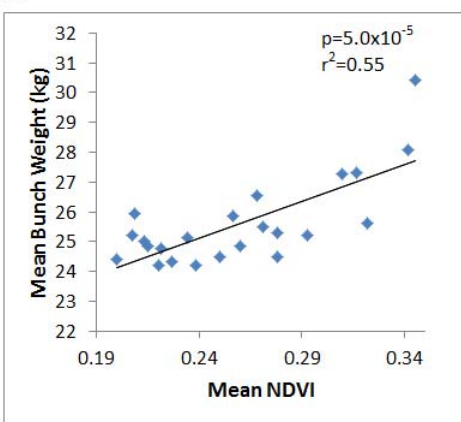

D

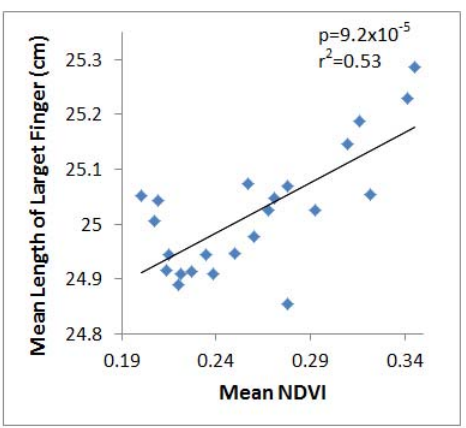

B

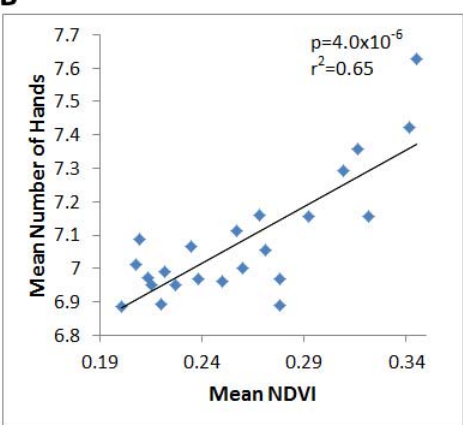

E

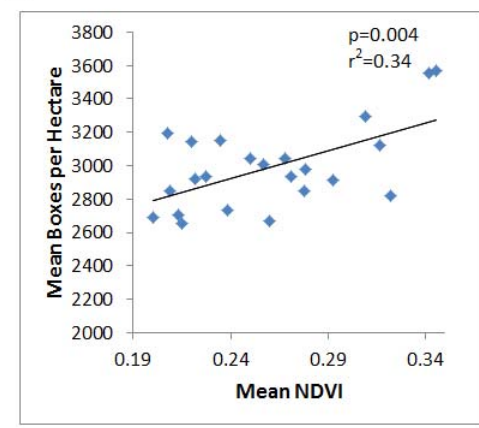

C

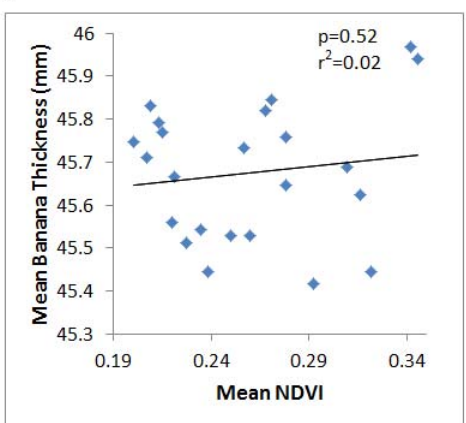

$\mathbf{F}$

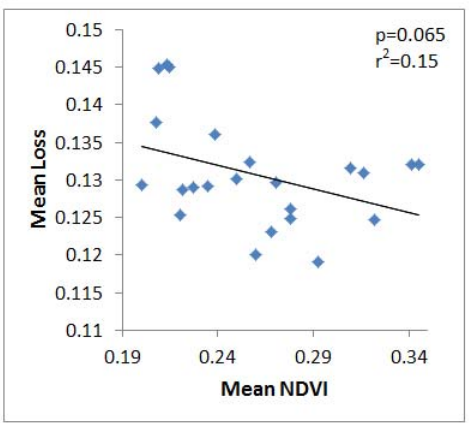

Fig. 3.10. Relationships among mean NDVI remotely sensed with Tetracam Micro and banana production measures: (a) Mean NDVI and Mean Bunch Weight; (b) Mean NDVI and Mean Number of Hands per Bunch; (c) Mean NDVI and Mean Banana Thickness on Second Hand; (d) Mean NDVI and Mean Length of Largest Finger; (e) Mean NDVI and Mean Boxes Per Hectare; (f) Mean NDVI and Mean Loss. 


\section{Acknowledgments}

This research could not have been performed without the funding by Dole Food

Company, Inc. or the support of its research team in Costa Rica. Special thanks are given to Jorge Gonzalez, Miguel Muñoz, Julian Gonzalez, and especially Hubert Gutierrez. I would also like to thank Ty Rozier of Elevated Horizons and Jesse Bushong and Brendan Doyle Wade of Marcus UAV for providing UAVs and mission support.

\section{Literature Cited}

Alexandratos, N., \& Bruinsma, J. (2012) World agriculture towards 2030/2050: the 2012 revision. In. Rome: FAO

Apan, A., Held, A., Phinn, S., \& Markley, J. (2004) Detecting sugarcane 'orange rust'disease using EO-1 Hyperion hyperspectral imagery. International Journal of Remote Sensing, 25, 489-498

Astorga, Y. (2005) The environmental impact of the banana industry. IBC II. Cited in Fairtrade Foundation, 2009, 9

Cassman, K.G. (1999) Ecological intensification of cereal production systems: Yield potential, soil quality, and precision agriculture. Proceedings of the National Academy of Sciences, 96, 5952-5959

Dobermann, A., \& Ping, J. (2004) Geostatistical integration of yield monitor data and remote sensing improves yield maps. Agronomy Journal, 96, 285-297

Evans, B.E. (2012) Banana Market. In. Gainesville, FL: Food and Resource Economics Department. Florida Cooperative Extension Service, Institute of Food and Agricultural Sciences, University of Florida

FAO (2009). Banana statistics Report to members and observers of the sub-group on bananas of the intergovernmental group on bananas and tropical fruits prepared by the Trade and Market Division, Food \& Agricultural Organization, Rome. . In

FAOSTAT (2014). http://faostat.fao.org/default.aspx 
Feeley, K.J., \& Machovina, B. (2014). Increasing preference for beef magnifies human impact on world's food web. Proceedings of the National Academy of Sciences, 111, E794-E794

Fiala, N. (2008). Meeting the demand: An estimation of potential future greenhouse gas emissions from meat production. Ecological Economics, 67, 412-419 http://dx.doi.org/10.1016/j.ecolecon.2007.12.021.

Florinsky, I.V. (1998). Combined analysis of digital terrain models and remotely sensed data in landscape investigations. Progress in Physical Geography, 22, 33-60

Foley, J.A., DeFries, R., Asner, G.P., Barford, C., Bonan, G., Carpenter, S.R., Chapin, F.S., Coe, M.T., Daily, G.C., Gibbs, H.K., Helkowski, J.H., Holloway, T., Howard, E.A., Kucharik, C.J., Monfreda, C., Patz, J.A., Prentice, I.C., Ramankutty, N., \& Snyder, P.K. (2005). Global Consequences of Land Use. Science, 309, 570-574

Foley, J.A., Ramankutty, N., Brauman, K.A., Cassidy, E.S., Gerber, J.S., Johnston, M., Mueller, N.D., O/'Connell, C., Ray, D.K., West, P.C., Balzer, C., Bennett, E.M., Carpenter, S.R., Hill, J., Monfreda, C., Polasky, S., Rockstrom, J., Sheehan, J., Siebert, S., Tilman, D., \& Zaks, D.P.M. (2011). Solutions for a cultivated planet. Nature, 478, 337-342.

http://www.nature.com/nature/journal/v478/n7369/abs/nature10452.html\#supplementaryinformation.

Frison, E., Escalant, J., Sharrock, S., Jain, S., \& Swennen, R. (2004). The global Musa genomic consortium: a boost for banana improvement. In, Banana improvement: cellular, molecular biology, and induced mutations. Proceedings of a meeting held in Leuven, Belgium, 24-28 September 2001. (pp. 341-349): Science Publishers, Inc.

Garnett, T. (2011). Where are the best opportunities for reducing greenhouse gas emissions in the food system (including the food chain)? Food Policy, 36, Supplement 1, S23-S32 http://dx.doi.org/10.1016/j.foodpol.2010.10.010.

Gebbers, R., \& Adamchuk, V.I. (2010). Precision agriculture and food security. Science, $327,828-831$

Godfray, H.C.J., Beddington, J.R., Crute, I.R., Haddad, L., Lawrence, D., Muir, J.F., Pretty, J., Robinson, S., Thomas, S.M., \& Toulmin, C. (2010). Food Security: The Challenge of Feeding 9 Billion People. Science, 327, 812-818

Goel, P., Prasher, S., Landry, J., Patel, R., Bonnell, R., Viau, A., \& Miller, J. (2003). Potential of airborne hyperspectral remote sensing to detect nitrogen deficiency and weed infestation in corn. Computers and Electronics in Agriculture, 38, 99-124

Hardin, P.J., \& Jensen, R.R. (2011). Small-scale unmanned aerial vehicles in environmental remote sensing: Challenges and opportunities. GIScience \& Remote Sensing, 48, 99-111 
Hillnhütter, C., Mahlein, A.-K., Sikora, R.A., \& Oerke, E.-C. (2012). Use of imaging spectroscopy to discriminate symptoms caused by Heterodera schachtii and Rhizoctonia solani on sugar beet. Precision Agriculture, 13, 17-32

Hirano, A., Welch, R., \& Lang, H. (2003). Mapping from ASTER stereo image data: DEM validation and accuracy assessment. ISPRS Journal of Photogrammetry and Remote Sensing, 57, 356-370

Hoffmann, C., \& Blomberg, M. (2004). Estimation of leaf area index of Beta vulgaris L. based on optical remote sensing data. Journal of agronomy and crop science, 190, 197 204

Inoue, Y., Morinaga, S., \& Tomita, A. (2000). A blimp-based remote sensing system for low-altitude monitoring of plant variables: a preliminary experiment for agricultural and ecological applications. International Journal of Remote Sensing, 21, 379-385

Jackson, R.D. (1986). Remote sensing of biotic and abiotic plant stress. Annual Review of Phytopathology, 24, 265-287

Jones, H., \& Schofield, P. (2008). Thermal and other remote sensing of plant stress. General Applied Plant Physiology, 34, 19-32

Jones, H.G. (1999). Use of infrared thermometry for estimation of stomatal conductance as a possible aid to irrigation scheduling. Agricultural and Forest Meteorology, 95, 139149

Jones, H.G. (2004). Application of thermal imaging and infrared sensing in plant physiology and ecophysiology. Advances in Botanical Research, 41, 107-163

Keyzer, M.A., Merbis, M., Pavel, I., \& Van Wesenbeeck, C. (2005). Diet shifts towards meat and the effects on cereal use: can we feed the animals in 2030? Ecological Economics, 55, 187-202

Knoth, C., Prinz, T., \& Loef, P. (2010). Microcopter-based Color Infrared (CIR) close range remote sensing as a subsidiary tool for precision farming. In, Proceedings of the Workshop on Remote Sensing Methods for Change Detection and Process Modelling (pp. 49-54)

Laliberte, A.S., Winters, C., \& Rango, A. (2011). UAS remote sensing missions for rangeland applications. Geocarto International, 26, 141-156

LDP, LLC. (2014) https://www.maxmax.com/endvi.htm.

Leon, C.T., Shaw, D.R., Cox, M.S., Abshire, M.J., Ward, B., Wardlaw III, M.C., \& Watson, C. (2003). Utility of remote sensing in predicting crop and soil characteristics. Precision Agriculture, 4, 359-384

Machovina, B., \& Feeley, K.J. (2013). Climate change driven shifts in the extent and location of areas suitable for export banana production. Ecological Economics, 95, 83-95 
Machovina, B., \& Feeley, K.J. (2014a). Meat consumption as a key impact on tropical nature: a response to Laurance et. al. Trends in Ecology \& Evolution, 29, 430

Machovina, B., \& Feeley, K.J. (2014b). Taking a Bite Out of Biodiversity. Science, 343, 838

Mahlein, A.-K., Steiner, U., Dehne, H.-W., \& Oerke, E.-C. (2010). Spectral signatures of sugar beet leaves for the detection and differentiation of diseases. Precision Agriculture, $11,413-431$

Manera, J.F., Rodríguez, L., Delrieux, C., \& Coppo, R. (2010). Aerial image acquisition and processing for remote sensing. Journal of Computer Science \& Technology, 10

Marín, D.H., Romero, R.A., Guzmán, M., \& Sutton, T.B. (2003). Black Sigatoka: an increasing threat to banana cultivation. Plant Disease, 87, 208-222

Mueller, N.D., Gerber, J.S., Johnston, M., Ray, D.K., Ramankutty, N., \& Foley, J.A. (2012). Closing yield gaps through nutrient and water management. Nature, 490, 254-257

Nellemann, C. (2009). The Environmental Food Crisis: The Environment's Role in Averting Future Food Crises: a UNEP Rapid Response Assessment. UNEP/Earthprint

Nutter Jr, F., Tylka, G., Guan, J., Moreira, A., Marett, C., Rosburg, T., Basart, J., \& Chong, C. (2002). Use of remote sensing to detect soybean cyst nematode-induced plant stress. Journal of Nematology, 34, 222

Plant, R.E. (2001). Site-specific management: the application of information technology to crop production. Computers and Electronics in Agriculture, 30, 9-29

Portillo, E. (2014). Chiquita loses \$25 million, citing shipping costs, Central American drought. In, Charlotte Observer

Pozdnyakova, L., Oudemans, P.V., Hughes, M.G., \& Giménez, D. (2002). Estimation of spatial and spectral properties of phytophthora root rot and its effects on cranberry yield. Computers and Electronics in Agriculture, 37, 57-70

Prabhakar, M., Prasad, Y., Thirupathi, M., Sreedevi, G., Dharajothi, B., \& Venkateswarlu, B. (2011). Use of ground based hyperspectral remote sensing for detection of stress in cotton caused by leafhopper (Hemiptera: Cicadellidae). Computers and Electronics in Agriculture, 79, 189-198

R Development Core Team (2014). R: A language and environment for statistical computing. In. Vienna, Austria: R Foundation for Statistical Computing

Rasband, W.S. (2014). ImageJ. In. Bethesda, Maryland, USA: U. S. National Institutes of Health

Ripple, W.J., Estes, J.A., Beschta, R.L., Wilmers, C.C., Ritchie, E.G., Hebblewhite, M., Berger, J., Elmhagen, B., Letnic, M., Nelson, M.P., Schmitz, O.J., Smith, D.W., Wallach, 
A.D., \& Wirsing, A.J. (2014a). Status and Ecological Effects of the World's Largest Carnivores. Science, 343

Ripple, W.J., Smith, P., Haberl, H., Montzka, S.A., McAlpine, C., \& Boucher, D.H. (2014b). Ruminants, climate change and climate policy. Nature Clim. Change, 4, 2-5

Robinson, J., \& Sauco, V. (2010). Bananas and Plantains. Oxfordshire, UK: CABI International

Steinfeld, H., Gerber, P., Wassenaar, T.D., Castel, V., \& Haan, C.d. (2006). Livestock's Long Shadow: Environmental Issues and Options. In. Rome: Food and Agriculture Organization of the United Nations

Steltzer, H., \& Welker, J.M. (2006). Modeling the effect of photosynthetic vegetation properties on the NDVI-LAI relationship. Ecology, 87, 2765-2772

Swain, K.C., Jayasuriya, H.P., \& Salokhe, V.M. (2007). Suitability of low-altitude remote sensing images for estimating nitrogen treatment variations in rice cropping for precision agriculture adoption. Journal of Applied Remote Sensing, 1, 013547-013547013511

Swain, K.C., Thomson, S.J., \& Jayasuriya, H.P. (2010). Adoption of an unmanned helicopter for low-altitude remote sensing to estimate yield and total biomass of a rice crop. Transactions of the ASABE, 53, 21-27

Takács, I.N.P.B.P., \& Tamás, J. Field size precision water management based on time series analysis of satellite images

Tamás, J., \& Lénárt, C. (2006). Analysis of a small agricultural watershed using remote sensing techniques. International Journal of Remote Sensing, 27, 3727-3738

Tilman, D. (1999). Global environmental impacts of agricultural expansion: the need for sustainable and efficient practices. Proceedings of the National Academy of Sciences, 96, $5995-6000$

Tilman, D., Fargione, J., Wolff, B., D'Antonio, C., Dobson, A., Howarth, R., Schindler, D., Schlesinger, W.H., Simberloff, D., \& Swackhamer, D. (2001). Forecasting Agriculturally Driven Global Environmental Change. Science, 292, 281-284

Turner, D., Lucieer, A., \& Watson, C. (2011). Development of an Unmanned Aerial Vehicle (UAV) for hyper resolution vineyard mapping based on visible, multispectral, and thermal imagery. In, Proceedings of 34th International Symposium on Remote Sensing of Environment (p. 4)

Veldkamp, E., Weitz, A., Staritsky, I., \& Huising, E. (1992). Deforestation trends in the Atlantic zone of Costa Rica: a case study. Land Degradation \& Development, 3, 71-84 
West, J.S., Bravo, C., Oberti, R., Lemaire, D., Moshou, D., \& McCartney, H.A. (2003). The potential of optical canopy measurement for targeted control of field crop diseases. Annual Review of Phytopathology, 41, 593-614

Wiley, J. (2008). The Banana: Empires, Trade Wars, and Globalization. . University of Nebraska Press

Worobetz, K. (2000). The growth of the banana industry in Costa Rica and its effect on biodiversity. Department of Biological Sciences, University of Alberta

Zarco-Tejada, P.J., Ustin, S., \& Whiting, M. (2005). Temporal and spatial relationships between within-field yield variability in cotton and high-spatial hyperspectral remote sensing imagery. Agronomy Journal, 97, 641-653

Zhang, C., \& Kovacs, J.M. (2012). The application of small unmanned aerial systems for precision agriculture: a review. Precision Agriculture, 13, 693-712

Zhang, M., Qin, Z., Liu, X., \& Ustin, S.L. (2003). Detection of stress in tomatoes induced by late blight disease in California, USA, using hyperspectral remote sensing. International Journal of Applied Earth Observation and Geoinformation, 4, 295-310 


\section{CHAPTER IV}

BIODIVERSITY CONSERVATION:

THE KEY IS REDUCING HUMAN CARNIVORY 


\begin{abstract}
Humans cause profound impacts on natural ecosystems, and our consumption of animal-sourced food products is one of our most powerful negative forces. Livestock production is the single largest driver of habitat loss, and both livestock and feedstock production are increasing in developing tropical countries where the majority of biological diversity resides. Livestock production is also a leading cause of climate change, compounding pressures on biodiversity. Reducing global animal product consumption should therefore be at the forefront of strategies aimed at reducing biodiversity loss. Such efforts would also impart positive impacts on human health through reduction of diseases of nutritional extravagance.
\end{abstract}

\title{
Introduction
}

Livestock production is the predominant driver of natural habitat loss worldwide. Over the 300 years ending in 1990, the extent of global cropland area increased more than five-fold and pasture areas increased more than six-fold, the latter encompassing an area 3.5 times larger than the United States. A direct cost of land being converted to food production was the loss of nearly one-half of all natural grasslands and the loss of nearly one-third of all natural forests worldwide (Goldewijk 2001). Although much of habitat lost to agriculture in the 1800 s was temperate forests and grasslands, the second half of the 1900s saw rapid agricultural expansion in tropical countries, predominantly at the expense of diverse tropical forests (Gibbs et al. 2010). Agricultural expansion is, by far, 
the leading cause of tropical deforestation (Geist and Lambin 2002). Although some agricultural expansion is driven by farmers growing crops for direct human consumption, livestock production accounts for approximately three-quarters of all agricultural land and nearly one-third of the ice-free land surface of the planet, making it the single largest anthropogenic land use type (Steinfeld et al. 2006a). Livestock comprise one-fifth of the total terrestrial biomass, and consume over half of directly-used human-appropriated biomass (Krausmann et al. 2008) and one-third of global cereal production (Alexandratos and Bruinsma 2012; Foley et al. 2011). Though difficult to quantify, animal product consumption by humans is likely the leading cause of modern species extinctions, since it is not only the major driver of deforestation but also a principle driver of land degradation, pollution, climate change, overfishing, sedimentation of coastal areas, facilitation of invasions by alien species, and killing of wild predators (Steinfeld et al. 2006a). Current global rates of extinction are about 1000 times the estimated background rate of extinction, (Pimm et al. 2014) and the number of species in decline are much higher in the tropics - even after accounting for the greater species diversity of the tropics (Dirzo et al. 2014). Here I present an overview of the connection between animal product consumption and current and likely future patterns of ecosystem degradation and biodiversity loss, the important influence of China in this relationship, the interwoven role of climate change, as well as the direct linkages with human health (Fig. 4.1). In addition, I propose solutions for potentially reducing the negative effects of animal product consumption on ecosystems, biodiversity, and human health. 


\section{Patterns of Biodiversity Loss Driven by Human Carnivory in the Tropics}

Animal product consumption is ubiquitous, but consumption levels, types and levels of livestock production, and future projected growth vary among Earth's tropical regions. The Amazon is the planet's largest continuous tropical forest and is a primary example of biodiversity loss being driven by livestock production. Never before has so much old-growth and primary forest been converted to human land uses so quickly as in the Amazon region (Walker et al. 2009). Nearly three-quarters of all deforested lands in the region have been converted to livestock pasture, and feedcrop production for domestic and international demand comprises much of the remaining deforested area (Nepstad et al. 2006; Nepstad et al. 2008; Walker et al. 2009). Rising worldwide demands for meat, feedcrops, and biofuel are driving rapid agro-industrial expansion into Amazon forest regions (Nepstad et al. 2008). Although there have been some recent brief periods when deforestation rates slowed in the Amazon as feedcrop (soy) production expanded more into pasture,(Macedo et al. 2012) rates have recently increased (INPE 2014), and feedcrop production as well as pasture is projected to continue expanding in the Amazon (Masuda and Goldsmith 2009). Eventually, cleared land that is suitable for feedstock soy production will become scarce and remaining forests outside of protected areas in the Brazilian Amazon will be at risk of conversion to soy (Nepstad et al. 2014). The woodland-savannah ecosystem of the Cerrado bordering the south-southeastern region of the Amazon is another expansive and diverse tropical habitat. More than half of the Cerrado's original expanse has already been converted to agriculture (Bianchi and Haig 2013), primarily for the production of beef and soy. At the current rate of loss, the entire 
two-million $\mathrm{km}^{2}$ of the Cerrado ecosystem ( $21 \%$ of Brazil's territory) could be altered in less than two decades (Steinfeld et al. 2006a). As another neotropical example, nearly half of Costa Rica's formerly highly-diverse tropical forests are now cleared and dedicated to livestock production (Morales-Hidalgo 2006). In fact, pasture is the top land use in Costa Rica, covering four times more land than is under protected status - this in a country often considered a model for biodiversity protection (Boza 1993). The conversion of forests to pasture in other Central American and Latin American nations has been similarly extensive (Szott et al. 2000).

In some other tropical areas there is little evidence of the livestock industry as a major factor in deforestation. For example, in Africa, timber harvesting and fire appear to be the two main processes leading to deforestation, with instances of farms replacing forest predominantly due to small-scale cropping (Steinfeld et al. 2006a). However, a rise in feedstock production is projected for Africa as international agricultural companies are acquiring or leasing land in Africa to grow feedstocks for export markets (Rulli et al. 2013), modeled after the industrial development of the Brazilian Cerrado region (Clements and Fernandes 2013). Hunting of wildlife as a direct meat source is often considered to be a more immediate and significant threat to the conservation of biological diversity in tropical forests than deforestation (Wilkie et al. 2005). The multibillion-dollar trade in bushmeat, especially critical in Africa, is among the most immediate threats to the persistence of tropical vertebrates (Brashares et al. 2004), which also causes many cascading trophic effects (Dirzo 2013; Ripple et al. 2014a). Hunting, habitat modification, and denial of access to water and other resources by humans, in 
combination with competition and disease transfer with livestock are driving critical decreases of wild ungulates in Africa (Daszak et al. 2000; Prins 2000).

Agricultural production in tropical Asia, which has transformed natural habitats for thousands of years, is based primarily around the intensive production of rice and wheat and other secondary crops. Multi-purpose livestock are integrated with many crops in small-scale, farming systems which characterize historical agriculture systems in Asia. This integration intensifies output, and the closed nature of these mixed farming systems makes them less damaging to the environment. However, in many Asian countries all of the available arable land is nearly completely utilized. In Southeast Asia, shifting cultivation is widely practiced and is associated with deforestation and erosion (Devendra and Thomas 2002a). Under growing demand by urbanizing populations, livestock production is rapidly changing in Asia, with both an increase of production and a shift away from mixed farming systems to intensive production systems located proximate to urban markets. This drives negative environmental consequences of increased monoculture feedstock demands at local and international scales as well as increased pollution of surface water, ground water and soils by nutrients, organic matter, and heavy metals (Rae and Hertel 2000) .

\section{Increasing Meat Production in Biodiverse Countries}

Because of its devastating effects on natural habitats and species, land-use change is projected to continue having the largest global impact on biodiversity, especially in 
tropical forests (Sala et al. 2000) where societies are increasing animal product and feedcrop production. The rapid expansion of livestock production in developing countries has been referred to as the "livestock revolution"' (Delgado 2003). As incomes in many developing countries have grown in recent decades, per capita consumption levels of animal products have also increased (Steinfeld et al. 2006b), including strong growth in the tropics (Figs. $4.2 \& 4.3$ ) (Tropics 2014). Half of global meat production now takes place in developing countries (Green et al. 2005), where annual per capita consumption of meat and use of cereals for animal feed doubled from $14 \mathrm{~kg}$ to $28 \mathrm{~kg}$ over two decades starting in 1980 (Delgado 2003; Steinfeld et al. 2006a). With continued economic growth, per capita meat consumption in some developing countries can be expected to quickly approach levels found in high-income industrialized countries of between $80 \mathrm{~kg}$ and $130 \mathrm{~kg} \mathrm{yr}^{1,}$ (Steinfeld et al. 2006b).

Animal products currently constitute a median of approximately $21 \%$ of the weight of global human diets - a $24 \%$ increase since the 1960 s. However, great disparity exists among developed and developing countries. Many developed countries have maintained high and stable animal product consumption rates constituting $40 \%$ or more of diets. This is contrasted with the majority of sub-Saharan countries and most of Southeast Asia which have had a pattern of low and stable animal product consumption rates $(<10 \%)$. Of concern are the historically-low, but increasing animal consumption rates found in several countries throughout Asia, Africa, and South America - most notably China which quadrupled its animal product consumption from $5 \%$ to $20 \%$ of diets since the 1960s (Bonhommeau et al. 2013). Increasing per capita consumption of 
animal products combined with rapidly growing populations in most developing countries will be a potent force driving habitat and biodiversity loss. Much of the future population growth will occur in biodiverse tropical nations. Today the tropics contain about $40 \%$ of global population, but house over half of all children under five. Within 40 years, it is expected that more than half the world's population will be in the tropics, containing over two-thirds of its young children, and adding 3 billion people by the end of the century (Tropics 2014).

Across global ecosystems, twenty-five biodiversity hotspots have been identified (Myers et al. 2000) that collectively contain as endemics approximately $44 \%$ of the world's plants and $35 \%$ of terrestrial vertebrates in an area that formerly covered only about $12 \%$ of the land surface of the planet. Due to human activities, the total extent of these hotspots has been reduced by nearly $90 \%$ of the original size - meaning that this wealth of biodiversity is now restricted to only $<2 \%$ of Earth's land surface. Among the top five hotspots for endemic diversity, the Caribbean retains only $11.3 \%$ of its primary vegetation, Madagascar 9.9\%, Sundaland 7.8\% and Brazil's Atlantic Forest 7.5\%. When analyzed by political boundaries, 17 megadiverse countries have been identified which collectively harbor the majority of the Earth's species (Mittermeier et al. 1997). Fifteen of the megadiverse countries are developing countries located in the tropics. Extrapolating rates of production of cattle, pigs, and chickens from 1985-2013 in these countries (Food and Agriculture Organization of the United Nations 2014) and the land area required to produce them (Röös et al. 2013) indicate that the developing tropical megadiverse countries will need to expand their agricultural areas by an estimated 3 million $\mathrm{km}^{2}$ over 
the next 35 years to meet projected increases in meat production (Fig. 4.4). Eleven of the tropical megadiverse countries have rates of increasing per capita meat (beef, pork, chicken) production (Fig. 4.5), and several of them (Ecuador, Brazil and China) are on trajectory to require by 2050 new areas of land for meat-production that are $>30 \%$ expansions of their total current agricultural areas. In the Philippines, the area of land required for future meat-production is projected to exceed $50 \%$ of the country's total current agricultural lands. To help meet these expansion needs, many developing countries are both acquiring land in other countries as well as selling or leasing land within their borders to fulfill other nation's food demands (Rulli et al. 2013).

The global increase in livestock production is destroying natural habitats and driving the loss of species at multiple trophic levels with cascading effects on biodiversity and ecosystem function. In a recent analysis of threats to the world's largest terrestrial carnivores (Ripple et al. 2014a), 94\% were found to be negatively affected by either habitat loss and/or persecution due to conflict with humans. Being the largest cause of global habitat loss, livestock are likely the most significant cause of the decline of large carnivores (Machovina and Feeley 2014c). Persecution of carnivores via shooting, trapping or poisoning is commonly a result of interactions with livestock. The loss of top predators can cause many negative trophic cascading effects within ecosystems (Ripple et al. 2014a). Grazing livestock can also cause more direct effects on entire ecosystems, such as riparian systems. For example, heavy grazing in riparian zones can lead to vegetation loss, soil erosion and reductions of fish and wildlife (Beschta et al. 2013). The conversion of forests into pasture and the industrial production of 
feedcrops also causes extensive soil erosion and downstream sedimentation of high diversity coastal habitats like coral reefs (Rogers 1990). Manure effluent and extensive over-use of fertilizers for feedstock production, especially corn (West et al. 2014), also pollute many waterways and are significant contributors to the more than 400 dead zones that exist at river mouths worldwide (Diaz and Rosenberg 2008).

\section{The Importance of China}

Because of changing dietary habits and increasing population densities, China will have especially profound future effects on biodiversity far beyond its own borders. From 2000-2030, China will likely add over 250 million new households, more than the total number of households in the entire Western Hemisphere in 2000 (Liu and Diamond 2005). Currently $20 \%$ of the weight of China's diet food consumption is animal productbased, approximating the global median, but consumption of animal products is on trajectory to reach $30 \%$ in 20 years (Bonhommeau et al. 2013; Food and Agriculture Organization of the United Nations 2014; Keyzer et al. 2005). Already over the past 20 years, animal products have increased from $10 \%$ to $20 \%$ of Chinese diets, and were only 5\% in 1960. Between 1978 and 2002, China's per capita consumption of meat, milk and eggs increased four-, four- and eight-fold, respectively (Liu and Diamond 2005).

Production within the nation has increased enormously over the past 50 years, with most growth occurring since the 1980s (Fig. 4.6) (Food and Agriculture Organization of the United Nations 2014). If China attains dietary habits similar to that of the United States

during the next 35 years, each of its projected 1.5 billion inhabitants would increase their 
consumption of meat and other animal-products by an average of 138\% (Bonhommeau et al. 2013; Liu and Diamond 2005).

Despite rising animal product demand, the extent of agricultural land in China has been decreasing under pressures of urbanization and land appropriation for mining, forestry and aquaculture. Furthermore, grasslands have been severely degraded by overgrazing and other pressures, with $90 \%$ of China's grasslands now considered degraded. Production rates of grasslands have decreased approximately $40 \%$ since the 1950s (Liu and Diamond 2005). Consequently, China’s increasing appetite for animal products will need to reach far beyond its own borders to meet its needs, importing both meat products and the feedstocks to produce meats locally (Rae and Hertel 2000). Much of the livestock production in China is fueled by soy-protein feedstock produced in the Amazon, with imports of soy from Brazil growing from zero in 1996 to approximately 7 million tons only ten years later. In 2003 China imported 21 million tons of soybeans, $10 \%$ of world production and $83 \%$ more than it imported in 2002 , with $29 \%$ of this soy coming from Brazil (Nepstad et al. 2006). In the 10 years from 2002 to 2012 this increased nearly $3 \mathrm{X}$ to reach 60 million tons (Fig. 4.7) (Food and Agriculture Organization of the United Nations 2014).

Land grabbing, the transfer of the right to own or use land from local communities to foreign investors through large-scale land acquisitions, has increased dramatically since 2005 . The increase began initially in response to the 2007-2008 global increase in food prices and growing food demand (especially in China and India). In 2010 the World Bank estimated that about 45 million hectares had been acquired by foreign 
investors since 2008 (Rulli et al. 2013). Grabbed areas are often in developing tropical countries with sufficient freshwater resources and can constitute a large fraction of a country's area (e.g. up to $19.6 \%$ in Uruguay, $17.2 \%$ in the Philippines, or $6.9 \%$ in Sierra Leone). Other tropical developing countries such as Liberia, Gabon, Papua New Guinea, Sierra Leone, and Mozambique have high grabbed-to-cultivated area ratios, indicating that the grabbed land may not have been cultivated before the acquisition but was developed through deforestation or land-use change (Hansen et al. 2010; Rulli et al. 2013).

Given current trends, the extent of land area converted to agriculture to meet growing global food demands is predicted to increase by approximately $18 \%$ from 2000 to 2050 . This equates to a loss of one billion ha of natural habitats - an area larger than the USA (Tilman et al. 2001). The globalization of food trade, production of foreign fodder sources, and standardization of food products is driving the replacement of wild and biodiversity-rich agriculture lands with extensive monoculture landscapes. Diversity found within traditional mix-cultured systems is threatened by this industrialization, including decreases in bees, butterflies, and plants (Idel and Reichert 2012). In addition, the biodiversity found within crops of traditional farming systems is decreasing as industrial agriculture expands (Altieri and Merrick 1987), driven by global demands for uniform products that ship and store well. 


\section{Livestock-Driven Climate Change}

Over the past 30 years, climate change has produced numerous shifts in the distributions and abundances of species, and its effects are projected to increase dramatically in the future (Walther et al. 2002), leading to potential declines or extinctions of many species (Carpenter et al. 2008; Keith et al. 2008; Pimm et al. 2014). One assessment of extinction risks for sample regions that cover $20 \%$ of the Earth's terrestrial surface indicated that $15-37 \%$ of species will be 'committed to extinction' by 2050 under mid-range climate-warming scenarios (Thomas et al. 2004). Effects on marine ecosystems already include decreased ocean productivity, altered food web dynamics, reduced abundances of habitat-forming species, shifting species distributions, and a greater incidence of diseases (Hoegh-Guldberg and Bruno 2010).

Given the potential widespread and profound effects of climate change, addressing the contribution of livestock-produced greenhouse gases is a valuable component of biodiversity conservation. Livestock are an important contributor to global warming through the production of greenhouse gases (carbon dioxide, methane, and nitrous oxide). Worldwide, the livestock sector is responsible for approximately $14.5 \%$ of all anthropogenic greenhouse gas emissions, approximately equivalent to emissions from the global transportation sector (Gerber et al. 2013). Land-use change (deforestation \& feedcrop expansion) dominates $\mathrm{CO}_{2}$ production from livestock with an estimated 2.4 billion tonnes of $\mathrm{CO}_{2}$ released annually (Steinfeld et al. 2006a). Releases of methane from enteric fermentation are equivalent to 2.2 billion tonnes of $\mathrm{CO}_{2}$. The use of nitrogen fertilizers in feed and manure production contribute $75-80 \%$ of annual agricultural 
emissions of $\mathrm{N}_{2} \mathrm{O}$, equivalent to 2.2 billion tonnes of $\mathrm{CO}_{2}$. Some data suggest that $\mathrm{N}_{2} 0$ is the largest livestock-driven climate change threat, primarily resulting from the production of manure and the intensive over-use of fertilizers for the production of animal feed (Idel and Reichert 2012). Indeed the amount of nitrogen produced by livestock via manure is estimated to exceed the global use of nitrogen fertilizers (Bouwman et al. 2009).

Land-use change involves not only the release of carbon with the conversion of forests and other habitats into grazing pastures, but also the conversion of natural grasslands into intensive feedcrop agriculture. Grasslands are one of the most extensive vegetation types, covering 15 million $\mathrm{km}^{2}$ in the tropics (as much as tropical forests) and another 9 million $\mathrm{km}^{2}$ in temperate regions (Scurlock and Hall 1998) for a total of nearly $40 \%$ of the world's land surface excluding Greenland and Antarctica (White et al. 2000). Grasslands are an important organic carbon store, with tropical savannahs alone holding $10-30 \%$ of the world's soil carbon. When grasslands are tilled for agriculture, large amounts of $\mathrm{CO}_{2}$ are released (Scurlock and Hall 1998). In a meta-analysis of carbon fluxes (Guo and Gifford 2002), it was found that shifts from pasture to crops always reduce soil carbon stocks by $50 \%$ or more, and in high rainfall environments the resultant soil carbon losses can exceed $75 \%$. Reverting croplands to grasslands reverses this process, eventually creating a carbon sink that can persist for up to many decades (McLauchlan et al. 2006). In the western hemisphere, over $70 \%$ of all grasslands have already been converted to croplands. In Asia and Africa over 19\% of grasslands have been converted to crops and in Oceania over $37 \%$ of grasslands have been converted to crops (White et al. 2000). Conversion of the world's remaining grasslands to agro- 
industrial croplands is likely to continue and potentially accelerate under ongoing international land grabbing and intensification of livestock production (Rulli et al. 2013).

\section{The Important Role of Ruminants}

There are a reported 3.6 billion domestic ruminants on Earth in 2011 (1.4 billon cattle, 1.1 billion sheep, 0.9 billion goats and 0.2 billon buffalo), and on average, 25 million domestic ruminants have been added to the planet each year over the past 50 years (Ripple et al. 2014b). Between 2000 and 2050, the global cattle population may increase from 1.5 billion to 2.6 billion, and the global goat and sheep population from 1.7 billion to 2.7 billion, increases of $73 \%$ and 59\%, respectively (Hubert et al. 2010). Distribution of ruminants across the earth overlaps extensively with areas that harbor high levels of biodiversity (Fig. 4.8). Of the considerable amount of greenhouse gases emitted by the livestock sector, $\mathrm{CO}_{2}$ from land-use change, methane production, and $\mathrm{N}_{2} \mathrm{O}$ production from ruminants are much higher than monogastrics (Fig. 4.9) (Ripple et al. 2014b). In addition to requiring the greatest area per kilogram of meat (or protein) produced of all types of livestock and globally occupying more area than any other land use, enteric fermentation from ruminant production alone is the largest source of anthropogenic methane emissions (Ripple et al. 2014b). Beef production also requires 6 times more reactive nitrogen to produce than dairy, poultry, pork, and eggs (Eshel et al. 2014). 


\section{Human Health}

In addition to ecological and biodiversity-related effects, increased animal product consumption also directly affects human health (Tilman and Clark 2014). For example, heart disease, the leading cause of human death, is strongly associated with the consumption of animal products, and can be largely prevented or reversed by switching to plant-based diets (Campbell and Campbell 2007; Campbell et al. 1998). Increased animal product consumption is closely tied to many 'diseases of nutritional extravagance' such as obesity and associated higher rates of heart disease, cancer, and diabetes, among other ailments (Lock et al. 2010; Menotti et al. 1999; Pan et al. 2013; Popkin et al. 2012). Under conditions of food abundance, diets based largely on plant foods are associated with health and longevity and shifts towards diets richer in animal products often leads to less-healthy populations (Nestle 1999). Studies have suggested that even small intakes of foods of animal origin are associated with significant plasma cholesterol concentrations, which are associated with significant increases in chronic degenerative disease mortality rates (Campbell and Junshi 1994). This has been evident with recent trends in China.

Diets of Chinese people that are higher in animal products are associated with increases in many diseases (Campbell and Campbell 2007; Campbell and Junshi 1994; Campbell et al. 1998; Popkin et al. 2012; Shu et al. 1993). Vegetarian, and especially vegan, diets can sometimes be deficient in B vitamins and some fatty acids, but these deficiencies can be addressed through small amounts of animal products (especially fish) in the diet, dietary diversity, or supplements (Davis and Kris-Etherton 2003). 


\section{Solutions}

Given that roughly 7.0 gigatons $(\mathrm{Gt})$ of plant biomass is required to produce the $0.26 \mathrm{Gt}$ of meat in our modern global agricultural systems (Smith et al. 2013), even a small increase in the consumption of animal-based foods will drive a large increase in habitat conversion and greenhouse gas emissions. I propose three solutions to help improve human nutritional health, decrease the land demands of agriculture, and protect plant and animal biodiversity: (1) reduce animal product consumption, (2) replace meat, and especially meat from ruminant sources, with more efficient protein sources, and (3) reintegrate livestock into diverse agricultural production systems.

\section{Reduce}

Eliminating the loss of energy available in plants via livestock production and instead growing crops only for direct human consumption is estimated to increase the number of food calories available for human consumption by as much as $70 \%$. This could feed an additional 4 billion people, exceeding the projected $2-3$ billion people to be added through future population growth (Cassidy et al. 2013). Substituting soy for meat as a source of protein for humans would reduce total biomass appropriation in 2050 by 94\% below 2000 baseline levels (Pelletier and Tyedmers 2010). Soy and other legumes are excellent sources of protein. When compared to an equivalent weight of common raw cuts of meats, soybeans contain on average twice the protein of beef, pork or chicken, and 10X more protein than whole milk (U.S. Department of Agriculture 2013). When comparing the area needed to produce $1 \mathrm{~kg}$ of protein from soybeans $\left(12 \mathrm{~m}^{2}\right)$ to the 
average land area required to produce common cuts of meat, chicken requires $3 \mathrm{X}$ more area $\left(39 \mathrm{~m}^{2}\right)$, pork 9X more area $\left(107 \mathrm{~m}^{2}\right)$, and beef $32 \mathrm{X}$ more area $\left(377 \mathrm{~m}^{2}\right)$ (Röös et al. 2013; U.S. Department of Agriculture 2013). A large amount of food, including animal products, is wasted worldwide. In the United States, 30\% of all food, worth more than US\$48 billion is thrown away each year (Nellemann 2009). Reducing this waste, especially related to animal product production, would impart large environmental benefits.

Traditional plant based diets combine legumes and grains (i.e. rice and soybeans in Asia, rice and black beans in Latin America) to achieve a complete and well-balanced source of amino acids for meeting human physiological requirements (Young and Pellett 1994). Although veganism is growing in popularity, eliminating animal based products from global diets is too simplistic and not practical (Idel and Reichert 2012). In smallscale farms, especially in poor cultures with marginal lands unsuitable for many agricultural crops, livestock are a valuable resource that converts low protein grass and other plants into more concentrated protein in a self-transportable format. For economically disadvantaged peoples, livestock can also provide draft power and a vital form of insurance during hard times (Laurance et al. 2014). However, low-cost, locallyavailable, and environmentally-sensitive practices and technologies can improve production (Pretty et al. 2003) of plant-based food sources and provide necessary caloric, protein, and nutrient levels (Campbell and Campbell 2007; Young and Pellett 1994) accentuated by small amounts of animal products. 
Based on a balance between the need to increase nutritional health (Campbell and Campbell 2007) and availability of calories with the need to decrease the land demands and ecological footprint of agriculture (Foley et al. 2011), I argue that people should strive toward a goal of significantly reducing the contribution of animal products in the human diet, ideally to a global average of $10 \%$ or less of calories (Machovina and Feeley 2014b; Machovina and Feeley 2014c). This is roughly equivalent to limiting average daily consumption of animal products to approximately 100 grams (a portion of meat approximately the size of a deck of playing cards or smaller). Others have proposed $90 \mathrm{~g}$ per day as a working global target (McMichael et al. 2007), shared more evenly among nations which currently range 10 -fold in meat consumption, with not more than $50 \mathrm{~g}$ per day coming from ruminants (McMichael et al. 2007). These scenarios, combined with further crop improvements, could enable the future global population to be fed on extant agricultural lands and potentially enable restoration of habitats (Machovina and Feeley 2014a; Machovina and Feeley 2014c). Reaching these goals and reducing the overall global animal product consumption to $\sim 10 \%$ will require significant decreases in per capita meat consumption by developed countries and little or no increase in most developing countries (Bonhommeau et al. 2013).

Success has previously been achieved in changing some dietary habits that are deleterious to the environment. A notable example is the recent campaign against consumption of shark fin soup in China. A large scale media campaign featuring Chinese National Basketball Association star Yao Ming in television, bus stop and billboard advertisements, and social media campaigns was disseminated widely throughout China 
in 2006 and again in 2009. Messages focused on the declining numbers of sharks and their important role in the ecosystem, the cruelty involved in the practice of finning, and the presence of mercury in shark fin soup. Survey's found that $83 \%$ of people exposed to the campaigns had stopped or reduced consumption (Fabinyi 2012). In 2012, the Chinese government pledged to ban shark fin soup from official banquets within three years. Conservation organization WildAid claims that there was a 50-70\% reduction in shark fin consumption over a two year period during the campaign (Denyer 2013). As with shark fin soup in China, animal product consumption is ingrained into many societies. High levels of livestock consumption are a traditional part of many diets or a sign of affluence in many countries. Meat is often believed (incorrectly) to be a physiologically necessary or superior form of protein. Many cultures also consider livestock ownership to be a sign of higher status (Laurance et al. 2014). In addition, government financial incentives often support livestock production (Geist and Lambin 2002; Nepstad et al. 2014) and animal product consumption over plant-based foods.

Clearly many challenges exist to reducing animal product consumption and increasing plant-based food consumption, but awareness is increasing. Fuelled by rapid urbanization, increases in animal-product consumption and lifestyle choices, chronic diseases have emerged as a critical public health issue in China, as they have in many other developing countries. The Chinese government has set a goal of promoting public health and making health care accessible and affordable for all Chinese citizens by year 2020 via the "Healthy China 2020" program. One important element of the program is to reduce chronic diseases by promoting healthy eating and active lifestyles (Hu et al. 
2011). These and other efforts to reduce animal product consumption on national and international levels will require significant political, financial, and cultural support.

\section{Replace}

Less than $5 \%$ of the protein and under $2 \%$ of the calories consumed by humans world-wide come from beef, compared to about $6 \%$ from pork, $6 \%$ from seafood, $9 \%$ poultry and eggs, and 10\% from milk (Boucher et al. 2012). However, the ecological footprint of beef is much higher than other meats. The type of livestock consumed has a strong influence on the area required for its production, and hence direct and indirect effects on biodiversity. Land-use rates vary by country (de Ruiter et al. 2014; Elferink and Nonhebel 2007) but feedstock-raised beef generally requires 2-3 times more area per kilogram produced than pork or chicken, and much greater area per unit of beef production is required on tropical pasture - up to 100 times greater than feedstock-raised animals (Cowan 1986). A recent analysis indicated that ruminants (primarily cattle) yield about 0.14 billion tons annually (measured as dry biomass) which is about the same as monogastric animal (mostly pigs and chickens). However, the ruminants require 20X more area to produce a ton of meat than chickens and pigs (28 hectares vs. 1.4 hectares). If cattle are raised only on feedcrops, the area of land required decreases to 2.8 hectares/ton but is still twice the area required for pigs or chickens (Smith et al. 2013).

Within a greater context of reducing the proportion of animal products in diets to $10 \%$ of calories, efforts to increase the proportion of chicken or pork while reducing beef consumption will magnify benefits to ecosystems and biodiversity. In addition to the 
lower amount of land required to produce meat, monogastrics produce a fraction of the methane as ruminants. Methane is the most abundant non- $\mathrm{CO}_{2}$ greenhouse gas and because it has a much shorter atmospheric lifetime ( $\sim 9$ years) than $\mathrm{CO}_{2}$ it holds the potential for more rapid reductions in radiative forcing. Decreases in worldwide ruminant populations could potentially be accomplished quickly and relatively inexpensively through meat-source replacement (Ripple et al. 2014b). A shift of preference for meat products is already occurring in many locations and should be further expanded. In developed countries, total livestock production increased by 22 percent between 1980 and 2004, but ruminant meat production declined by 7 percent while that of poultry and pigs increased by 42 percent. As a result, the share of production of poultry and pigs has gone up from 59 to 69 percent of total meat production. Poultry is the meat commodity with the highest growth rates across the world. With increased awareness about health effects of ruminant consumption, shifts in dietary preferences occur. For example, per capita retail beef demand in the United States declined by nearly 50\% from 1980 to 1998, offset largely by increased chicken consumption (Food and Agriculture Organization of the United Nations 2014).

Providing economical alternative protein sources, either plant-based, lowfootprint animal product (chicken, aquaculture fish, or insect) to developing countries can also help relieve pressures on hunting of wildlife as a protein source. In one study in Ghana, fish supplies, which could vary $24 \%$ between consecutive years, were negatively correlated with biomass of terrestrial mammals, indicating a transfer of harvest pressure and consumption between these resources. Developing cheap protein alternatives to 
bushmeat as well as improving fisheries management to avert extinctions of tropical wildlife is critical (Brashares et al. 2004). However, unsustainable consumption of wildlife also remains a problem even in many relatively prosperous countries with sufficient protein supplies as consumption of bushmeat in many locations is considered a delicacy, sign of affluence, or "badge of honor" (Bennett 2013). This is similar to the historical and cultural perceptions around shark-fin soup in China which, as discussed above, has been addressed with considerable success through public awareness campaigns.

\section{Reintegrate}

A major ongoing trend in livestock production is the intensification of production systems through industrial-scale feedcrop production and confined livestock production in high capacity facilities. Confined livestock production systems in industrialized countries are the source of much of the world's poultry and pig meat production, and such systems are being established in developing countries, particularly in Asia, to meet increasing demand (Thornton 2010) with at least $75 \%$ of total production growth to 2030 projected to occur in confined systems (Bruinsma 2003). Traditional fibrous feedcrops are in relative decline, and protein-rich feeds together with nutritional additives that enhance feed conversion are on the rise (Steinfeld et al. 2006b). As global livestock production grows and intensifies, it depends less on locally-available feed resources but increasingly on feed concentrates that are traded domestically and internationally. In 2004, a total of 690 million tonnes of cereals were fed to livestock (34\% of the global cereal harvest) and another 18 million tonnes of oilseeds (mainly soy). In addition, 295 
million tonnes of protein-rich processing by-products were used as feed (mainly bran, oilcakes and fish meal) (Steinfeld et al. 2006b).

Intensification of livestock operations is being supported by intensification of crop production systems. From 1980 to 2004, the total global supply of cereals increased by $46 \%$ while the area dedicated to cereal production shrank by $5.2 \%$ (Steinfeld et al. 2006a). In some areas the intensification of global livestock production combined with yield increases have reduced some pressure to expand livestock industries into natural areas. For example, from 2006 to 2010, deforestation in the Amazon frontier state of Mato Grosso decreased to 30\% of its average from 1996 to 2005, and $78 \%$ of production increases in soy were due to expansion ( $22 \%$ to yield increases), with $91 \%$ on previouslycleared land (Macedo et al. 2012). However, deforestation rates in the Brazilian Amazon have recently increased (INPE 2014).

Although the land footprint of feedcrop-produced beef can be as low as one-tenth the area required by pasture-raised beef (Smith et al. 2013), or even 100 times less than some low-productivity tropical pasture beef (Cowan 1986), many negative tradeoffs result from intensive agriculture since it is highly dependent on non-renewable fossil fuel energy to produce fertilizers, biocides, and operate machinery that exacerbates climate change. Increased nutrient pollution from farms and confined operations, $\mathrm{N}_{2} \mathrm{O}$ and ammonia production, soil erosion, and biocide contamination are all results of livestock industry intensification (Steinfeld et al. 2006a). Within the context of reducing animal product consumption (ideally to $10 \%$ of diets), and replacing much of the high environmental-footprint ruminant production with monogastric or other low-impact 
protein production, intensification is an additional, but not optimal solution. With the release of highly-productive arable lands that would occur with reduction of meat consumption and replacement of ruminants, an opportunity exists to reintegrate livestock production into agricultural systems that are designed around the structure and processes of natural ecosystems. Much of Asia's traditional agricultural systems have operated in this fashion for thousands of years (Devendra and Thomas 2002a, b), and this agricultural philosophy is the basis of modern permaculture (Mollison 1988; Mollison and Holmgren 1979).

In contrast to modern intensive livestock production, within a permaculture system, livestock are integrated into a designed and diverse agricultural production system that strives to maximize production of foods from solar (not fossil fuel) energy, conserve nutrients and water, and produce little waste. Livestock are integrated as herbivores or omnivores would be in a natural ecosystem, consuming a variety of feeds, and producing nutrient-rich waste that is returned into the system. In addition to providing food for humans, livestock provide many services within the system. For example, in addition to being fed grains, chickens can be utilized in movable zones to prepare fields for planting. This "chicken tractor" produces eggs and meat, turns the surface of the soil, removes insects and other pests, and deposits nutrients. Permaculture systems are designed to best fit into local ecological limitations and opportunities.

The closed-system, diverse, coupled designs of permaculture systems are reflected in traditional integrated agriculture-aquaculture (IAA) systems of Asia (Prein 2002), which supply diets traditionally based primarily on consumption of fruits, 
vegetables, and whole grains with small amounts of animal products (Campbell and Campbell 2007). These systems are based on multiple synergies in which outputs from sub-systems in an integrated farming system become inputs to other sub-systems instead of being wasted. The flow and reuse of energy and nutrients between enterprises produces higher efficiency outputs while reducing external energy or nutrient inputs. Many types of IAA systems exist such as the rice-aquaculture systems from which fish, freshwater prawns and crabs, snails, mussels and frogs are harvested, and which may be fertilized with agricultural or human waste. For example, in the Mekong Delta of Vietnam, fruit orchards are built upon berms dug from adjacent canals that provide fish habitat and connect to nearby rice fields. Fish and freshwater prawns can move between the sub-systems and benefit from the decomposing rice straw as well as fruit and insects dropping into the water. Due to energetic efficiencies of fish metabolism and the use of energy and nutrient inputs that are often wasted or not utilized in modern high production livestock systems, IAA systems can have very high productivities. The area required to produce $1 \mathrm{~kg}$ of fish is as small as $1.25 \mathrm{~m}^{2}$ to $2 \mathrm{~m}^{2}$ (Prein 2002), which is much less than area required to produce beef $\left(67.8 \mathrm{~m}^{2}\right)$, pork $\left(19.2 \mathrm{~m}^{2}\right)$, chicken $\left(6.9 \mathrm{~m}^{2}\right)$ (Röös et al. 2013), or even soybeans (4.3 $\left.\mathrm{m}^{2}\right)$ (Masuda and Goldsmith 2009).

\section{Conclusions}

Given the large ecological footprint of livestock production, humans' negative impact on biodiversity can be significantly reduced by: (1) reducing demand for animalbased food products and increasing proportions of plant-based foods in diets; (2) 
replacing ecologically-inefficient ruminants and bushmeat with monogastrics, aquaculture, or other more-efficient protein sources; and (3) reintegrating livestock production away from single-product, intensive, fossil-fuel based systems into diverse, coupled systems designed more closely around the structure and functions of ecosystems that conserve energy and nutrients. Applying ecologically-integrated structures and functions to plant and livestock production systems to support a future with lower animal-product food demands would drastically reduce habitat and biodiversity loss, fossil fuel energy use, greenhouse gas emissions, and pollution while providing highly nutritious diets that greatly improve global human health. 


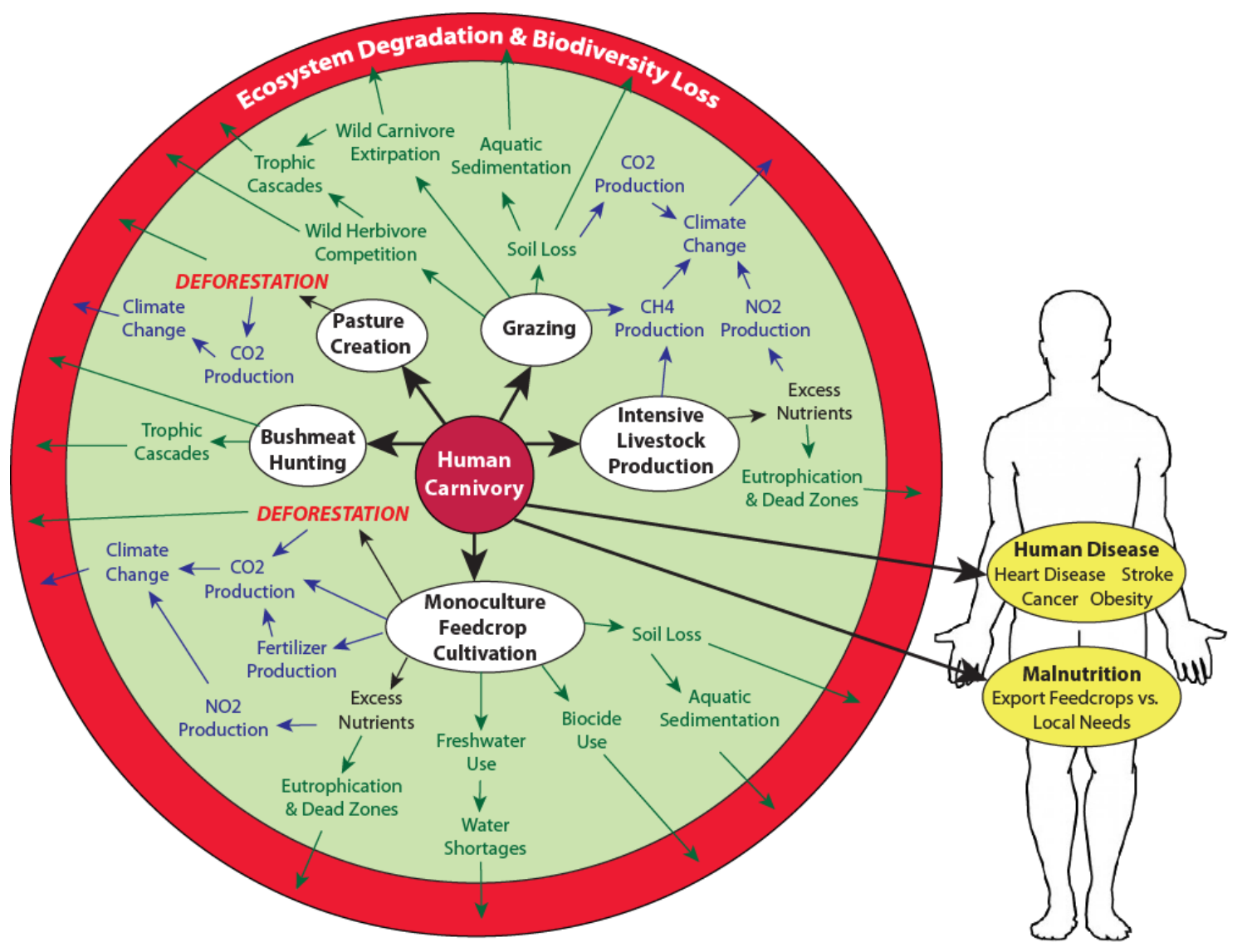

Fig. 4.1. The diverse and interrelated negative effects of human carnivory on ecosystems, biodiversity, and human health. Climate change effects are highlighted in blue whereas direct ecosystem effects are highlighted in green. Bushmeat hunting directly reduces threatened animal populations (Brashares et al. 2004) which has negative cascading effects on ecosystems (Dirzo 2013; Ripple et al. 2014a). Pasture creation is a powerful cause of deforestation and CO2 release (Nepstad et al. 2006; Nepstad et al. 2008; Walker et al. 2009). Grazing livestock compete with wild herbivores (Prins 2000) as well as instigate extirpation of predators by ranchers, both which cause cascading ecosystem effects (Ripple et al. 2014a). Grazing causes soil loss, downstream sedimentation of aquatic habitats, $\mathrm{CO} 2$ loss from soils, as well as enteric $\mathrm{CH} 4$ production (Steinfeld et al. 2006a). Intensive (industrial) livestock production releases $\mathrm{CH} 4$ and produces nutrientrich wastes, which cause eutrophication of aquatic ecosystems as well as NO2 releases (Rae and Hertel 2000; Steinfeld et al. 2006a). The cultivation of feedcrops for intensive livestock production has many negative effects, primarily deforestation and habitat change that also releases CO2 (Scurlock and Hall 1998; Steinfeld et al. 2006a; Steinfeld et al. 2006b). The production of fertilizers releases CO2, and their excessive use releases NO2 and also causes eutrophication of aquatic habitats (Steinfeld et al. 2006a) . Feedcrop cultivation competes for freshwater sources, extensively uses biocides, and causes large amounts of soil loss, all which negatively affect surrounding ecosystems (Steinfeld et al. 2006a). The consumption of animal products by humans is an underlying cause of the top causes of death of people, including heart disease (\#1 cause of death), strokes (\#2 
cause of death), many cancers, and is also a contributor to the global rise of obesity and its associated ailments (Campbell and Campbell 2007; Campbell and Junshi 1994;

Campbell et al. 1998; Lock et al. 2010; Menotti et al. 1999; Pan et al. 2013; Popkin et al. 2012; Tilman and Clark 2014). International land and water grabbing for the production of feedcrops can cause smallholder agriculture to lose access to land and water, increasing local populations dependency on food aid and international food subsidies (Rulli et al. 2013). 



Fig. 4.2. Human Trophic Level (HTL) of megadiverse countries based on consumption of livestock products in 1961 and 2009 (Bonhommeau et al. 2013). An HTL of 2 indicates $0 \%$ of a nation's diet is composed of animal products whereas an HTL of 2.5 indicates $50 \%$ of a nation's diet composed of animal products. The blue line indicates the global median value of 2.21. Eleven of the 16 megadiverse countries represented here have increased HTLs from 1961 to 2009. (Data not available for Papua New Guinea, which is ranked among the 17 megadiverse countries)(Myers et al. 2000). Consumption of bushmeat, especially important in Africa, was not included in analysis. 


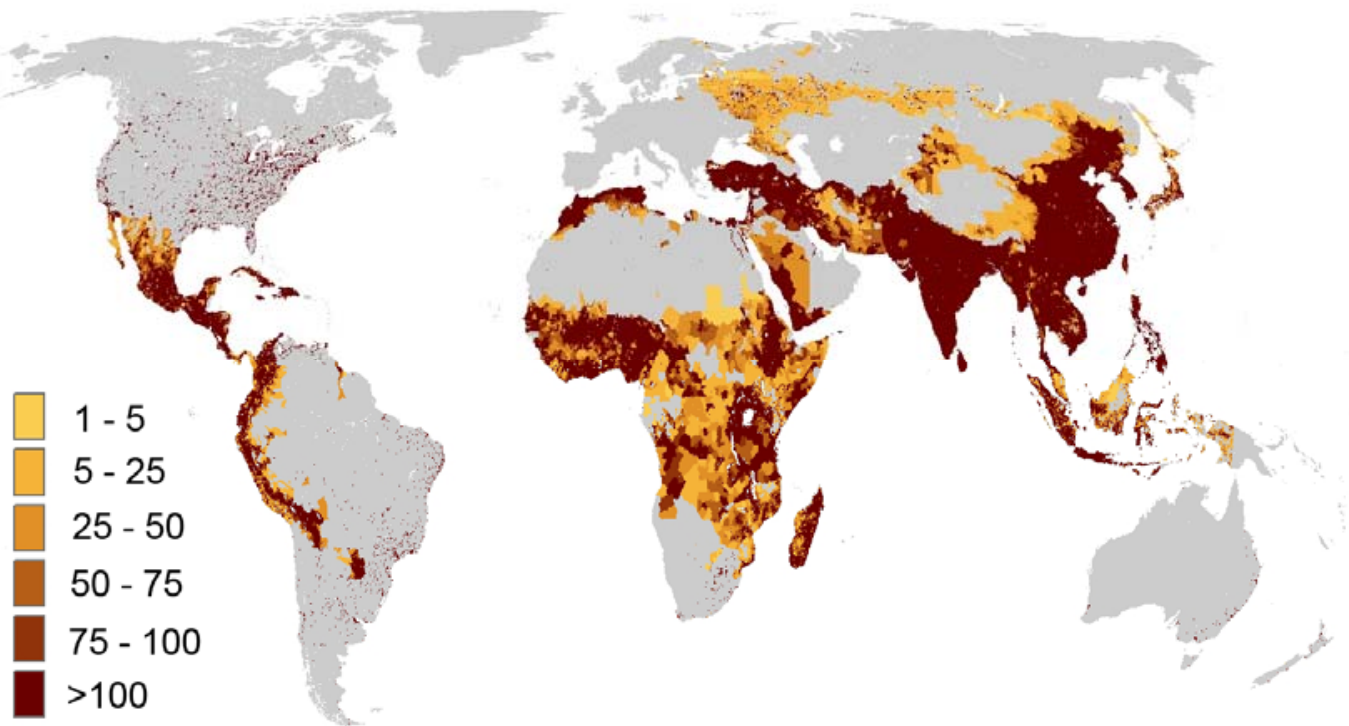

Fig. 4.3. Map showing projected global increases of demand for meat (beef, pig, chicken) from 2000-2030. Legend indicates $\mathrm{kg} / \mathrm{km}^{2}$ demand increase (FAO 2011). Developing countries of Latin America, Africa, and Asia exhibit the highest levels of demand increase. Data for Europe not available. 
A.

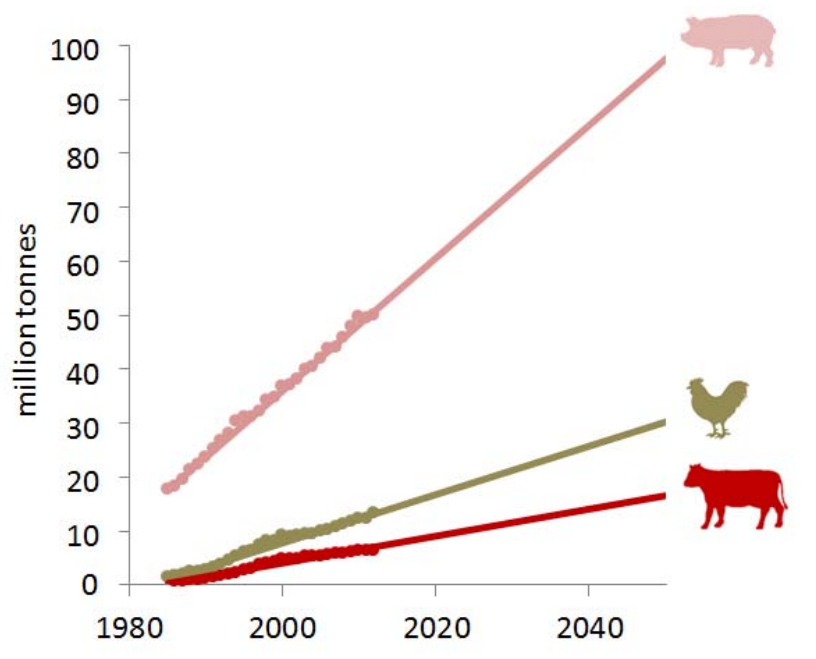

C.
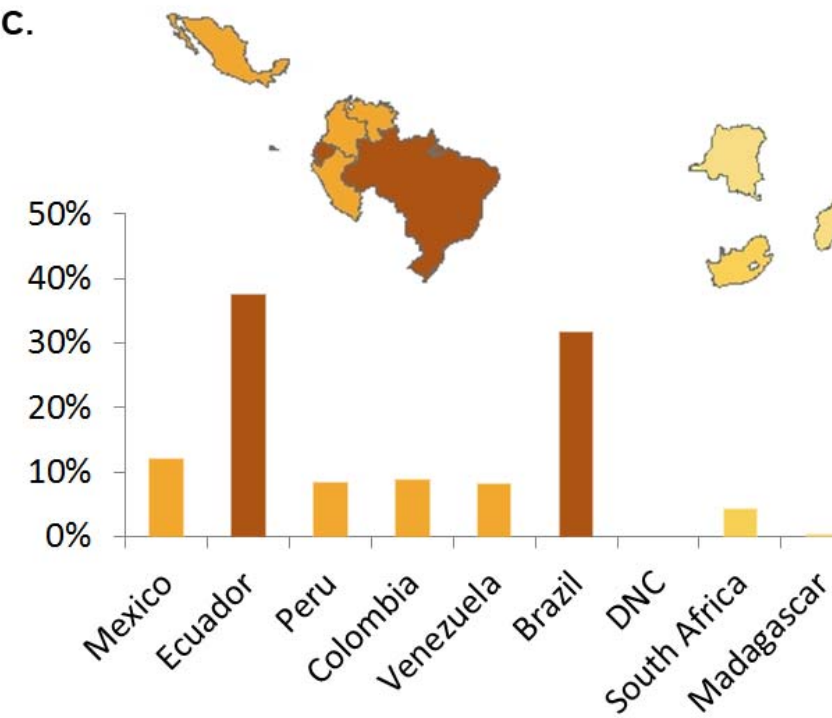

B.
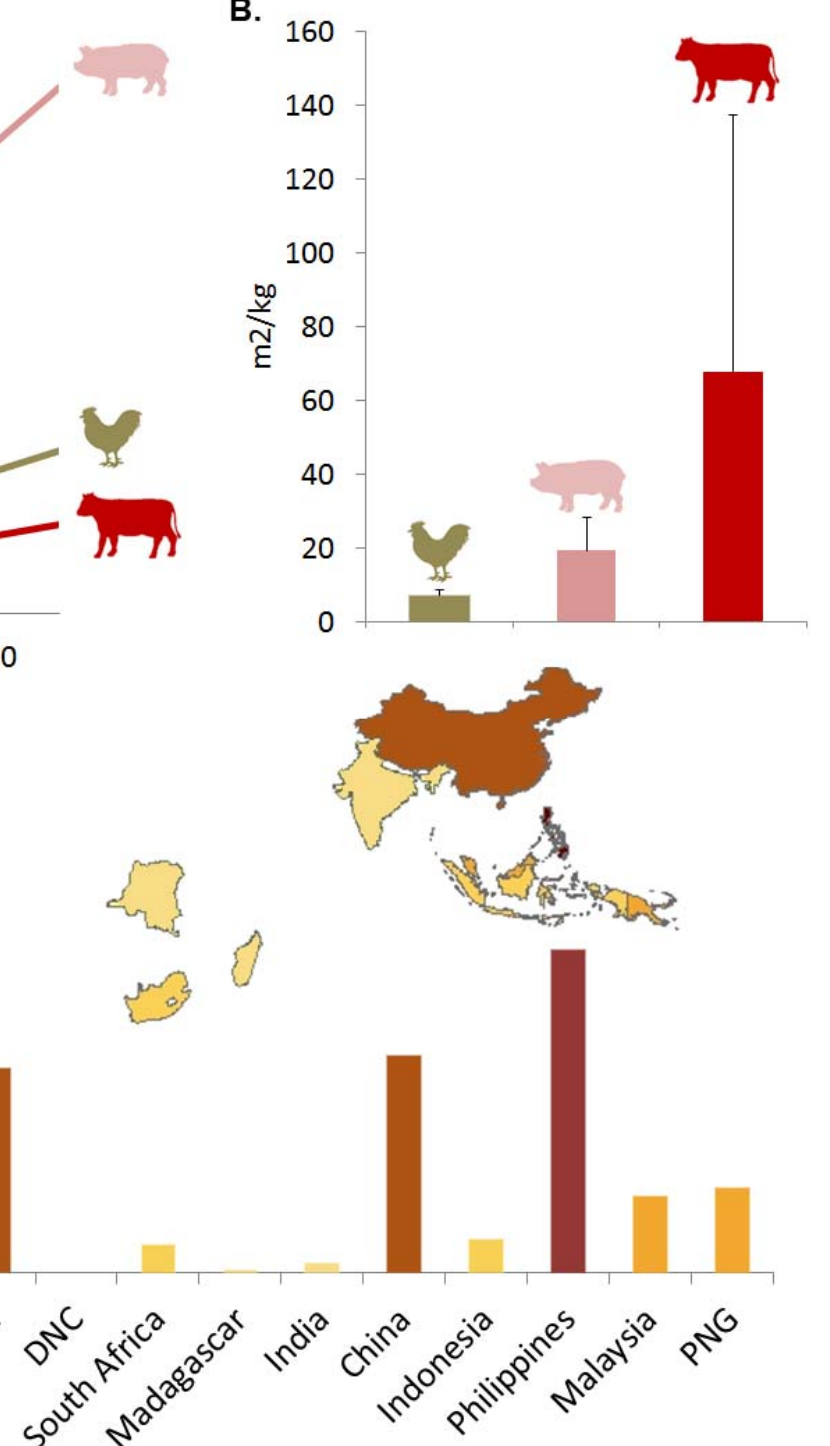

Fig 4.4. Projected increases in area required to produce meat in developing megadiverse (DMD) countries by 2050. (a) Extrapolating recent (1985-2012) production data for beef, chicken, and pork (Food and Agriculture Organization of the United Nations 2014) in each DMD country to 2050 (data for China shown) multiplied by (b) mean area required to produce livestock biomass (Röös et al. 2013) provides (c) an estimate of area in each country required to produce livestock in 2050 as a percentage increase beyond total current agricultural area (2012)(Food and Agriculture Organization of the United Nations 2014). Agricultural area expansion needs can be met by internal expansion or by agricultural expansion in other countries and importation of feedcrops and/or meat products. This analysis addresses only beef, chicken, and pork. It does not include milk, eggs, other meat sources, or dairy, which would increase area projections. 


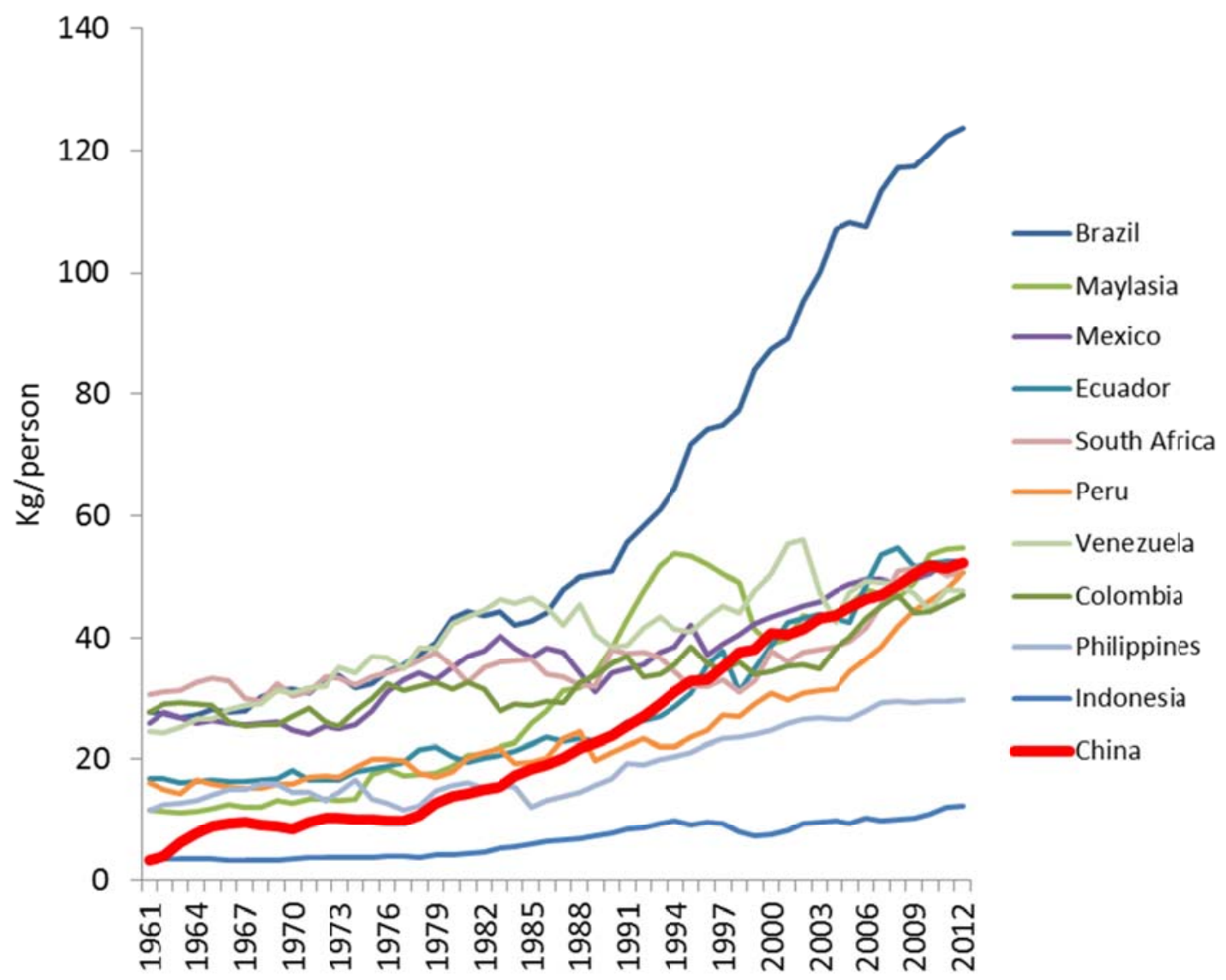

Fig. 4.5. Developing megadiverse (DMD) countries with increasing rates of per capita meat (chicken, pork, beef) production. Eleven of 15 DMD countries have increasing rates of per capita meat production (chicken, pork, beef)(Food and Agriculture Organization of the United Nations 2014). The strong growth of China (highlighted) is of special note as its population ( 1.377 billion in 2012 ) is $59 \%$ larger than the combined populations (868 million) (Food and Agriculture Organization of the United Nations 2014) of the other 10 DMD countries with increasing rates of meat production. 


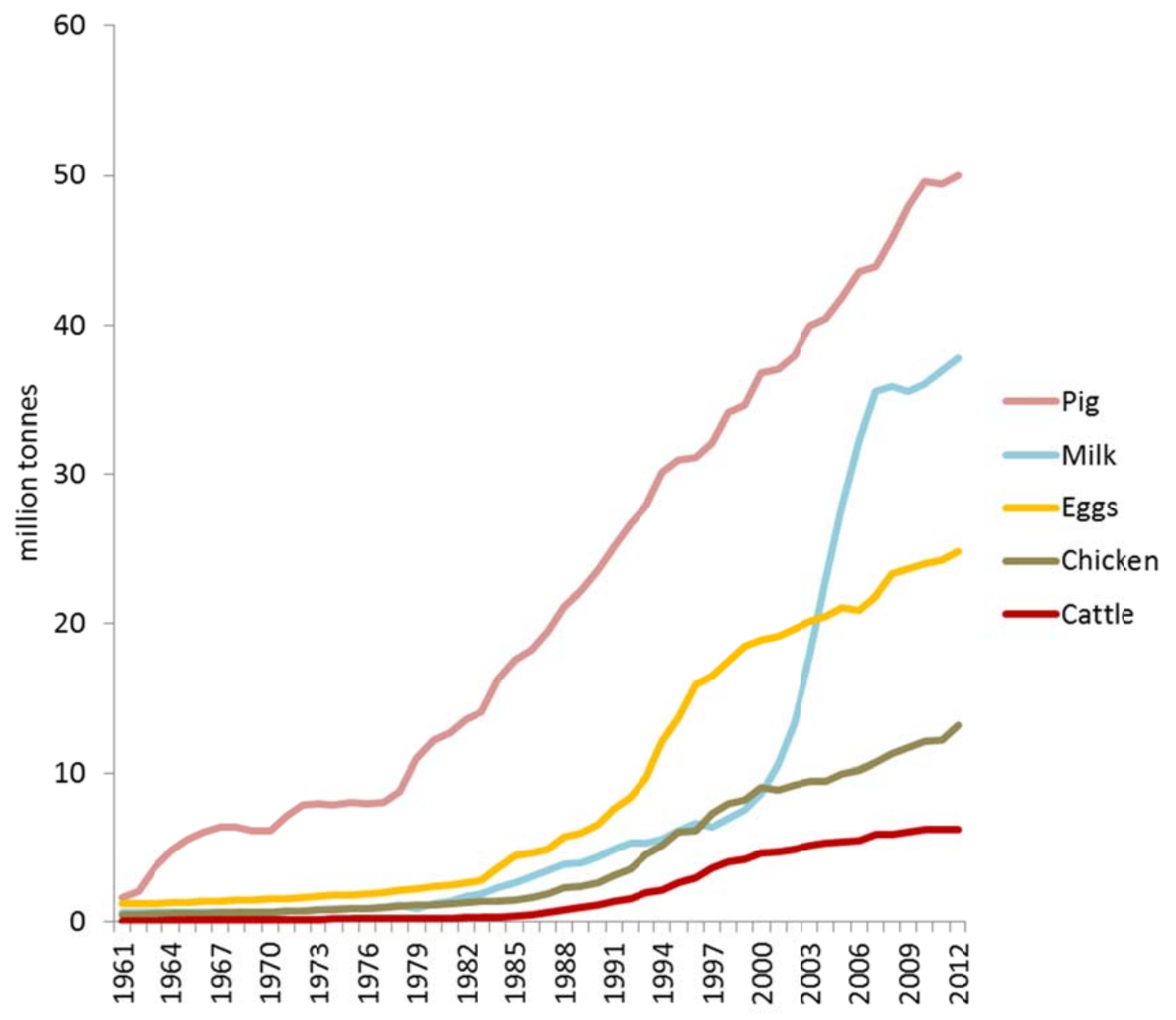

Fig. 4.6. Animal product production in China 1961-2012. Production of animal products in China has increased dramatically, especially since the 1980s (Food and Agriculture Organization of the United Nations 2014). 




Fig. 4.7. Production, exports and imports of soybeans for Brazil and China 1964-2012 (Food and Agriculture Organization of the United Nations 2014). Much of China's demand for feedcrops is driving deforestation and land-use change outside of its national boundaries, including within the Brazilian Amazon. Production of soy within China has increased minimally to meet growing Chinese demand for livestock feed, whereas imports have increased dramatically. Approximately half of Brazil's production is used internally, and half exported as feedcrops for markets around the world, including the European Union and China. 


\section{Birds}



Mammals

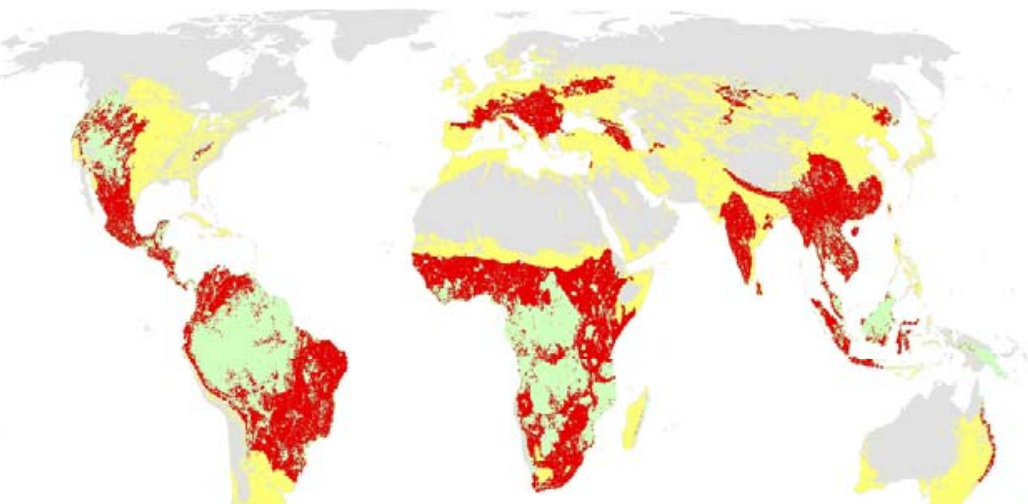

Amphibians

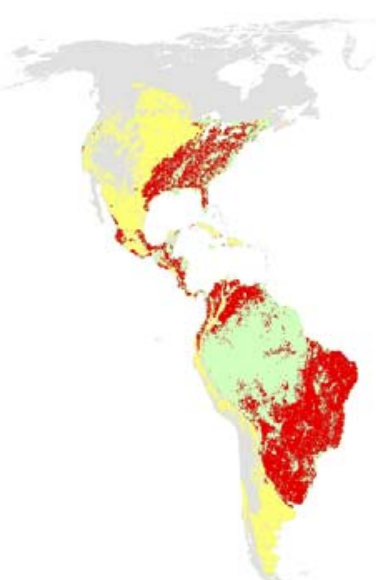

Low Ruminant, Low Richness Low Ruminant, High Richness

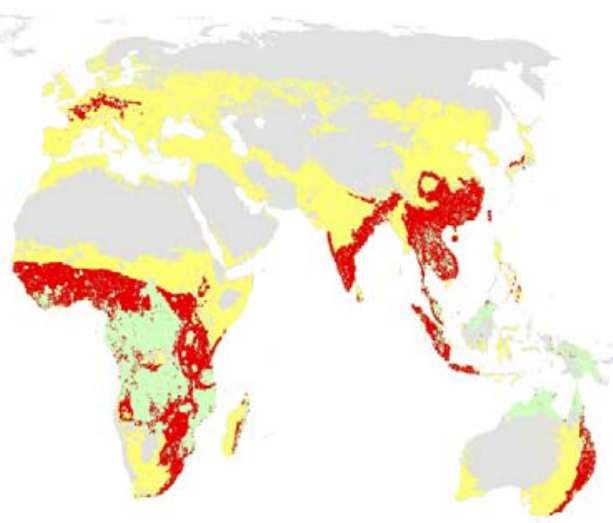

High Ruminant, Low Richness

High Ruminant, High Richness 
Fig 4.8. Maps indicating density (high or low) of ruminants (cattle, goats, sheep) (Wint and Robinson 2007) and species richness (high or low) of birds, mammals, and amphibians (Pimm et al. 2014) . Classification as 'high' indicate values above the mean value for all areas and 'low' indicate values below the mean value. Mean density value for ruminants $=5 / \mathrm{km}^{2}$. Mean species richness values $\left(\mathrm{spp} / 100 \mathrm{~km}^{2}\right)$ are: birds $=192$, mammals $=56$, amphibians $=16$. 


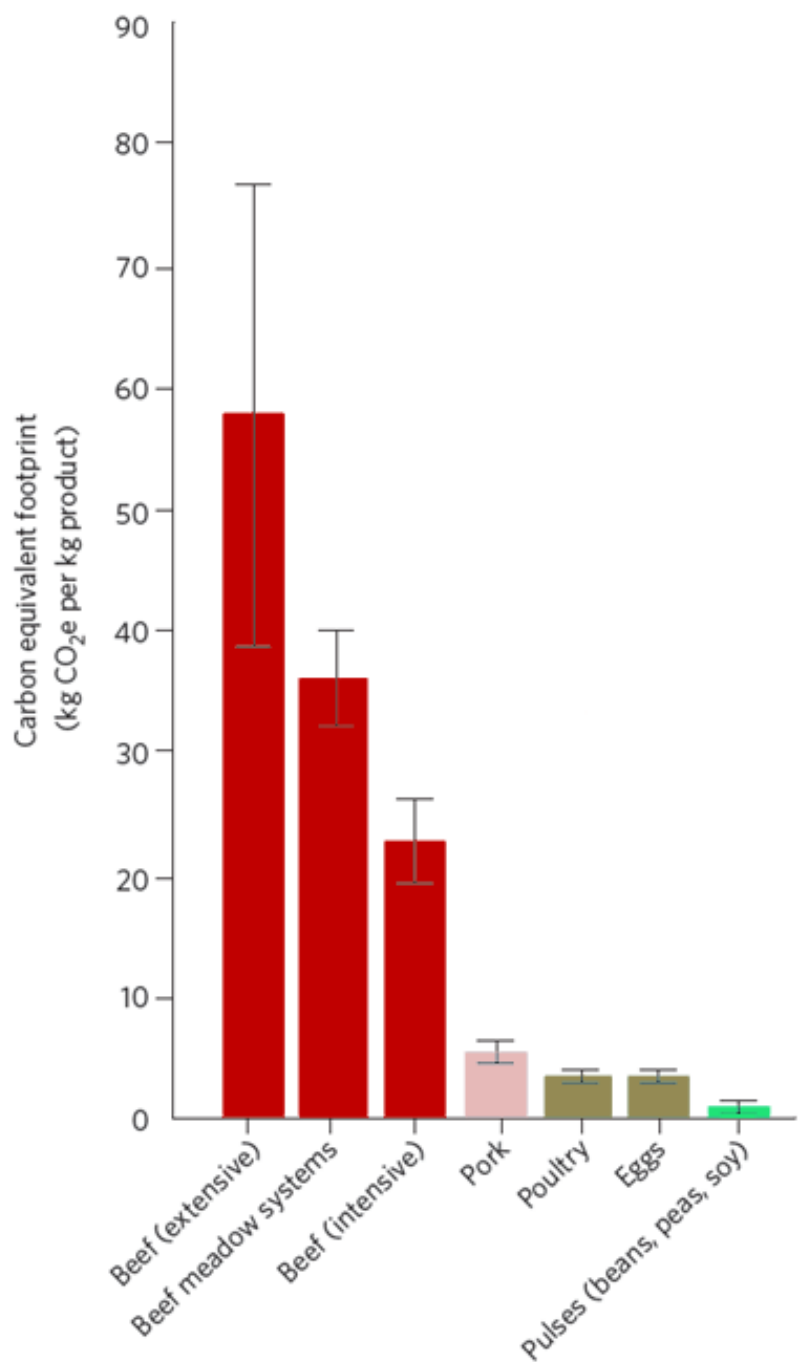

Fig 4.9. Average carbon equivalent footprint of meats and pulses per kilogram of product from a global meta-analysis of life-cycle assessment studies (adapted from Ripple et al. 2014)(Ripple et al. 2014b). Extensive beef involves cattle grazing across large pastoral systems, whereas intensive beef typically involves feedlots. Error bars represent standard errors. 


\section{Literature Cited}

Alexandratos, N., \& Bruinsma, J. (2012) World agriculture towards 2030/2050: the 2012 revision. In. Rome: FAO

Altieri, M.A., \& Merrick, L. (1987) In situ conservation of crop genetic resources through maintenance of traditional farming systems. Economic Botany, 41, 86-96

Bennett, E.L. (2013) Hunting for sustainability in tropical forests. Columbia University Press

Beschta, R.L., Donahue, D.L., DellaSala, D.A., Rhodes, J.J., Karr, J.R., O’Brien, M.H., Fleischner, T.L., \& Williams, C.D. (2013) Adapting to climate change on western public lands: addressing the ecological effects of domestic, wild, and feral ungulates.

Environmental Management, 51, 474-491

Bianchi, C.A., \& Haig, S.M. (2013) Deforestation trends of tropical dry forests in central Brazil. Biotropica, 45, 395-400

Bonhommeau, S., Dubroca, L., Le Pape, O., Barde, J., Kaplan, D.M., Chassot, E., \& Nieblas, A.-E. (2013) Eating up the world's food web and the human trophic level. Proceedings of the National Academy of Sciences, 110, 20617-20620

Boucher, D.H., Pipa, E., Goodman, L., May-Tobin, C., Mulik, K., \& Roquemore, S. (2012) Grade A choice? Solutions for deforestation-free meat. In. Cambridge, Massachussetts: Union of Concerned Scientists

Bouwman, A., Beusen, A., \& Billen, G. (2009) Human alteration of the global nitrogen and phosphorus soil balances for the period 1970-2050. Global Biogeochemical Cycles, 23

Boza, M.A. (1993) Conservation in Action: Past, Present, and Future of the National Park System of Costa Rica. Conservation Biology, 7, 239-247

Brashares, J.S., Arcese, P., Sam, M.K., Coppolillo, P.B., Sinclair, A.R., \& Balmford, A. (2004) Bushmeat hunting, wildlife declines, and fish supply in West Africa. Science, 306, 1180-1183

Bruinsma, J. (2003) World agriculture: towards 2015/2030. An FAO perspective. Earthscan Publications Ltd

Campbell, T.C., \& Campbell, T.M. (2007) The China study: the most comprehensive study of nutrition ever conducted and the startling implications for diet, weight loss and long-term health. Wakefield Press

Campbell, T.C., \& Junshi, C. (1994) Diet and chronic degenerative diseases: perspectives from China. The American Journal of Clinical Nutrition, 59, 1153S-1161S 
Campbell, T.C., Parpia, B., \& Chen, J. (1998) Diet, lifestyle, and the etiology of coronary artery disease: the Cornell China Study. The American Journal of Cardiology, 82, 18-21

Carpenter, K.E., Abrar, M., Aeby, G., Aronson, R.B., Banks, S., Bruckner, A., Chiriboga, A., Cortés, J., Delbeek, J.C., \& DeVantier, L. (2008) One-third of reef-building corals face elevated extinction risk from climate change and local impacts. Science, 321, 560563

Cassidy, E., West, P.C., Gerber, J.S., \& Foley, J.A. (2013) Redefining agricultural yields: from tonnes to people nourished per hectare. Environmental Research Letters, 8, 8

Clements, E.A., \& Fernandes, B.M. (2013) Land grabbing, agribusiness and the peasantry in Brazil and Mozambique. Agrarian South: Journal of Political Economy, 2, 41-69

Cowan, R.S. (1986) Beef and Dairy Production in the Wet Tropics. In, Pasture Management for the Wet Tropics of South East Asia Course Manual (pp. 193-197): 6th FAO Regional Training course

Daszak, P., Cunningham, A.A., \& Hyatt, A.D. (2000) Emerging infectious diseases of wildlife--threats to biodiversity and human health. Science, 287, 443-449

Davis, B.C., \& Kris-Etherton, P.M. (2003) Achieving optimal essential fatty acid status in vegetarians: current knowledge and practical implications. The American Journal of Clinical Nutrition, 78, 640S-646S

de Ruiter, H., Kastner, T., \& Nonhebel, S. (2014) European dietary patterns and their associated land use: Variation between and within countries. Food Policy, 44, 158-166

Delgado, C.L. (2003) Rising consumption of meat and milk in developing countries has created a new food revolution. The Journal of Nutrition, 133, 3907S-3910S

Denyer, S. (2013) In China, victory for wildlife conservation as citizens persuaded to give up shark fin soup. In, Washington Post

Devendra, C., \& Thomas, D. (2002a) Crop-animal systems in Asia: importance of livestock and characterisation of agro-ecological zones. Agricultural Systems, 71, 5-15

Devendra, C., \& Thomas, D. (2002b) Smallholder farming systems in Asia. Agricultural Systems, 71, 17-25

Diaz, R.J., \& Rosenberg, R. (2008) Spreading dead zones and consequences for marine ecosystems. Science, 321, 926-929

Dirzo, R. (2013) Defaunation, alteration of trophic cascades and extinction of ecological processes: Consequences for ecosystems and humans. In, New Frontiers in Tropical Biology: The Next 50 Years (A Joint Meeting of ATBC and OTS): Atbc 
Dirzo, R., Young, H.S., Galetti, M., Ceballos, G., Isaac, N.J.B., \& Collen, B. (2014)

Defaunation in the Anthropocene. Science, 345, 401-406

Elferink, E.V., \& Nonhebel, S. (2007) Variations in land requirements for meat production. Journal of Cleaner Production, 15, 1778-1786

Eshel, G., Shepon, A., Makov, T., \& Milo, R. (2014) Land, irrigation water, greenhouse gas, and reactive nitrogen burdens of meat, eggs, and dairy production in the United States. Proceedings of the National Academy of Sciences

Fabinyi, M. (2012) Historical, cultural and social perspectives on luxury seafood consumption in China. Environmental Conservation, 39, 83-92

FAO (2011) Mapping supply and demand for animal-source foods to 2030, by T.P Robinson \& F. Pozzi. Animal Production and Health Working Paper. No. 2. Rome. In: FAO

Foley, J.A., Ramankutty, N., Brauman, K.A., Cassidy, E.S., Gerber, J.S., Johnston, M., Mueller, N.D., O/'Connell, C., Ray, D.K., West, P.C., Balzer, C., Bennett, E.M., Carpenter, S.R., Hill, J., Monfreda, C., Polasky, S., Rockstrom, J., Sheehan, J., Siebert, S., Tilman, D., \& Zaks, D.P.M. (2011) Solutions for a cultivated planet. Nature, 478, $337-342$

Food and Agriculture Organization of the United Nations (2014) FAOSTAT. In. Rome, Italy: FAO

Geist, H.J., \& Lambin, E.F. (2002) Proximate Causes and Underlying Driving Forces of Tropical Deforestation Tropical forests are disappearing as the result of many pressures, both local and regional, acting in various combinations in different geographical locations. Bioscience, 52, 143-150

Gerber, P.J., Steinfeld, H., Henderson, B., Mottet, A., Opio, C., Dijkman, J., Falcucci, A., \& Tempio, G. (2013) Tackling climate change through livestock: a global assessment of emissions and mitigation opportunities. Food and Agriculture Organization of the United Nations (FAO)

Gibbs, H.K., Ruesch, A.S., Achard, F., Clayton, M.K., Holmgren, P., Ramankutty, N., \& Foley, J.A. (2010) Tropical forests were the primary sources of new agricultural land in the 1980s and 1990s. Proceedings of the National Academy of Sciences, 107, 1673216737

Goldewijk, K.K. (2001) Estimating global land use change over the past 300 years: The HYDE Database. Global Biogeochemical Cycles, 15, 417-433

Green, R.E., Cornell, S.J., Scharlemann, J.P.W., \& Balmford, A. (2005) Farming and the Fate of Wild Nature. Science, 307, 550-555 
Guo, L.B., \& Gifford, R. (2002) Soil carbon stocks and land use change: a meta analysis. Global Change Biology, 8, 345-360

Hansen, M.C., Stehman, S.V., \& Potapov, P.V. (2010) Quantification of global gross forest cover loss. Proceedings of the National Academy of Sciences, 107, 8650-8655

Hoegh-Guldberg, O., \& Bruno, J.F. (2010) The Impact of Climate Change on the World's Marine Ecosystems. Science, 328, 1523-1528

Hu, F.B., Liu, Y., \& Willett, W.C. (2011) Preventing chronic diseases by promoting healthy diet and lifestyle: public policy implications for China. Obesity Reviews, 12, $552-559$

Hubert, B., Rosegrant, M., van Boekel, M.A., \& Ortiz, R. (2010) The future of food: scenarios for 2050. Crop Science, 50, S-33-S-50

Idel, A., \& Reichert, T. (2012) Livestock production and food security in a context of climate change, and environmental and health challenges. Environment Review

INPE (2014) INPE divulga resultado final do PRODES 2013. In

Keith, D.A., Akçakaya, H.R., Thuiller, W., Midgley, G.F., Pearson, R.G., Phillips, S.J., Regan, H.M., Araújo, M.B., \& Rebelo, T.G. (2008) Predicting extinction risks under climate change: coupling stochastic population models with dynamic bioclimatic habitat models. Biology Letters, 4, 560-563

Keyzer, M.A., Merbis, M., Pavel, I., \& Van Wesenbeeck, C. (2005) Diet shifts towards meat and the effects on cereal use: can we feed the animals in 2030? Ecological Economics, 55, 187-202

Krausmann, F., Erb, K.-H., Gingrich, S., Lauk, C., \& Haberl, H. (2008) Global patterns of socioeconomic biomass flows in the year 2000: A comprehensive assessment of supply, consumption and constraints. Ecological Economics, 65, 471-487

Laurance, W.F., Sayer, J., \& Cassman, K.G. (2014) The impact of meat consumption on the tropics: reply to Machovina and Feeley. Trends in Ecology \& Evolution

Liu, J., \& Diamond, J. (2005) China's environment in a globalizing world. Nature, 435, $1179-1186$

Lock, K., Smith, R.D., Dangour, A.D., Keogh-Brown, M., Pigatto, G., Hawkes, C., Fisberg, R.M., \& Chalabi, Z. (2010) Health, agricultural, and economic effects of adoption of healthy diet recommendations. The Lancet, 376, 1699-1709

Macedo, M.N., DeFries, R.S., Morton, D.C., Stickler, C.M., Galford, G.L., \& Shimabukuro, Y.E. (2012) Decoupling of deforestation and soy production in the southern Amazon during the late 2000s. Proceedings of the National Academy of Sciences, 109, 1341-1346 
Machovina, B., \& Feeley, K.J. (2014a) Livestock: limit red meat consumption. Nature, $508,186-186$

Machovina, B., \& Feeley, K.J. (2014b) Meat consumption as a key impact on tropical nature: a response to Laurance et. al. Trends in Ecology \& Evolution, 29, 430

Machovina, B., \& Feeley, K.J. (2014c) Taking a Bite Out of Biodiversity. Science, 343, 838

Masuda, T., \& Goldsmith, D. (2009) World Soybean Production: Area Harvested, Yield, and Long-Term Projections. International Food and Agribusiness Management Review, 12

McLauchlan, K.K., Hobbie, S.E., \& Post, W.M. (2006) Conversion From Agriculture To Grassland Builds Soil Organic Matter On Decadal Timescales. Ecological Applications, $16,143-153$

McMichael, A.J., Powles, J.W., Butler, C.D., \& Uauy, R. (2007) Food, livestock production, energy, climate change, and health. The Lancet, 370, 1253-1263

Menotti, A., Kromhout, D., Blackburn, H., Fidanza, F., Buzina, R., \& Nissinen, A. (1999) Food intake patterns and 25-year mortality from coronary heart disease: cross-cultural correlations in the Seven Countries Study. European Journal of Epidemiology, 15, 507515

Mittermeier, R.A., Gil, P.R., \& Mittermeir, C.G. (1997) Megadiversity. Earth's biologically wealthiest nations. In, CEMEX. Mexico City, Mexico

Mollison, B. (1988) Permaculture: a designer's manual. Permaculture: a designer's manual.

Mollison, B.C., \& Holmgren, D. (1979) Permaculture. Transworld Publishers

Morales-Hidalgo, D. (2006) Tree Cover Assessment: With Special Focus on the Relative Position Issue; Case Studies in Open Areas in Costa Rica. Cuvillier Verlag

Myers, N., Mittermeier, R.A., Mittermeier, C.G., da Fonseca, G.A.B., \& Kent, J. (2000) Biodiversity hotspots for conservation priorities. Nature, 403, 853-858

Nellemann, C. (2009) The Environmental Food Crisis: The Environment's Role in Averting Future Food Crises: a UNEP Rapid Response Assessment. UNEP/Earthprint

Nepstad, D., McGrath, D., Stickler, C., Alencar, A., Azevedo, A., Swette, B., Bezerra, T., DiGiano, M., Shimada, J., Seroa da Motta, R., Armijo, E., Castello, L., Brando, P., Hansen, M.C., McGrath-Horn, M., Carvalho, O., \& Hess, L. (2014) Slowing Amazon deforestation through public policy and interventions in beef and soy supply chains. Science, 344, 1118-1123 
Nepstad, D.C., Stickler, C.M., \& Almeida, O.T. (2006) Globalization of the Amazon Soy and Beef Industries: Opportunities for Conservation. Conservation Biology, 20, 15951603

Nepstad, D.C., Stickler, C.M., Filho, B.S.-., \& Merry, F. (2008) Interactions among Amazon land use, forests and climate: prospects for a near-term forest tipping point. Philosophical Transactions of the Royal Society B: Biological Sciences, 363, 1737-1746

Nestle, M. (1999) Animal v. plant foods in human diets and health: is the historical record unequivocal? Proceedings of the Nutrition Society, 58, 211-218

Pan, A., Sun, Q., Bernstein, A.M., Manson, J.E., Willett, W.C., \& Hu, F.B. (2013) Changes in red meat consumption and subsequent risk of type 2 diabetes mellitus: three cohorts of US men and women. JAMA internal medicine, 173, 1328-1335

Pelletier, N., \& Tyedmers, P. (2010) Forecasting potential global environmental costs of livestock production 2000-2050. Proceedings of the National Academy of Sciences, 107, 18371-18374

Pimm, S.L., Jenkins, C.N., Abell, R., Brooks, T.M., Gittleman, J.L., Joppa, L.N., Raven, P.H., Roberts, C.M., \& Sexton, J.O. (2014) The biodiversity of species and their rates of extinction, distribution, and protection. Science, 344

Popkin, B.M., Adair, L.S., \& Ng, S.W. (2012) Global nutrition transition and the pandemic of obesity in developing countries. Nutrition Reviews, 70, 3-21

Prein, M. (2002) Integration of aquaculture into crop-animal systems in Asia. Agricultural Systems, 71, 127-146

Pretty, J.N., Morison, J.I., \& Hine, R.E. (2003) Reducing food poverty by increasing agricultural sustainability in developing countries. Agriculture, Ecosystems \& Environment, 95, 217-234

Prins, H.H. (2000) Competition between wildlife and livestock in Africa. Wildlife conservation by sustainable use (pp. 51-80): Springer

Rae, A.N., \& Hertel, T.W. (2000) Future developments in global livestock and grains markets: the impacts of livestock productivity convergence in Asia-Pacific. Australian Journal of Agricultural and Resource Economics, 44, 393-422

Ripple, W.J., Estes, J.A., Beschta, R.L., Wilmers, C.C., Ritchie, E.G., Hebblewhite, M., Berger, J., Elmhagen, B., Letnic, M., Nelson, M.P., Schmitz, O.J., Smith, D.W., Wallach, A.D., \& Wirsing, A.J. (2014a) Status and Ecological Effects of the World's Largest Carnivores. Science, 343

Ripple, W.J., Smith, P., Haberl, H., Montzka, S.A., McAlpine, C., \& Boucher, D.H. (2014b) Ruminants, climate change and climate policy. Nature Clim. Change, 4, 2-5 
Rogers, C.S. (1990) Responses of coral reefs and reef organisms to sedimentation. Marine ecology progress series. Oldendorf, 62, 185-202

Röös, E., Sundberg, C., Tidåker, P., Strid, I., \& Hansson, P.-A. (2013) Can carbon footprint serve as an indicator of the environmental impact of meat production? Ecological Indicators, 24, 573-581

Rulli, M.C., Saviori, A., \& D’Odorico, P. (2013) Global land and water grabbing. Proceedings of the National Academy of Sciences, 110, 892-897

Sala, O.E., Stuart Chapin , F., III, Armesto, J.J., Berlow, E., Bloomfield, J., Dirzo, R., Huber-Sanwald, E., Huenneke, L.F., Jackson, R.B., Kinzig, A., Leemans, R., Lodge, D.M., Mooney, H.A., Oesterheld, M.n., Poff, N.L., Sykes, M.T., Walker, B.H., Walker, M., \& Wall, D.H. (2000) Global Biodiversity Scenarios for the Year 2100. Science, 287, $1770-1774$

Scurlock, J.M.O., \& Hall, D.O. (1998) The global carbon sink: a grassland perspective. Global Change Biology, 4, 229-233

Shu, X.O., Zheng, W., Potischman, N., Brinton, L.A., Hatch, M.C., Gao, Y.-T., \& Fraumeni, J.F. (1993) A population-based case-control study of dietary factors and endometrial cancer in Shanghai, People's Republic of China. American Journal of Epidemiology, 137, 155-165

Smith, P., Haberl, H., Popp, A., Erb, K.h., Lauk, C., Harper, R., Tubiello, F.N., Siqueira Pinto, A., Jafari, M., \& Sohi, S. (2013) How much land-based greenhouse gas mitigation can be achieved without compromising food security and environmental goals? Global Change Biology, 19, 2285-2302

Steinfeld, H., Gerber, P., Wassenaar, T.D., Castel, V., \& Haan, C.d. (2006a) Livestock's Long Shadow: Environmental Issues and Options. In. Rome: Food and Agriculture Organization of the United Nations

Steinfeld, H., Wassenaar, T., \& Jutzi, S. (2006b) Livestock production systems in developing countries: status, drivers, trends. Rev. sci. tech. Off. int. Epiz, 25, 505-516

Szott, L., Ibrahim, M., \& Beer, J. (2000) The hamburger connection hangover: cattle, pasture land degradation and alternative land use in Central America. Bib. Orton IICA/CATIE

Thomas, C.D., Cameron, A., Green, R.E., Bakkenes, M., Beaumont, L.J., Collingham, Y.C., Erasmus, B.F., De Siqueira, M.F., Grainger, A., \& Hannah, L. (2004) Extinction risk from climate change. Nature, 427, 145-148

Thornton, P.K. (2010) Livestock production: recent trends, future prospects.

Philosophical Transactions of the Royal Society B: Biological Sciences, 365, 2853-2867 
Tilman, D., \& Clark, M. (2014) Global diets link environmental sustainability and human health. Nature, advance online publication

Tilman, D., Fargione, J., Wolff, B., D'Antonio, C., Dobson, A., Howarth, R., Schindler, D., Schlesinger, W.H., Simberloff, D., \& Swackhamer, D. (2001) Forecasting Agriculturally Driven Global Environmental Change. Science, 292, 281-284

Tropics, S.o.t. (2014) State of the Tropics 2014 Report. In. Cairns, Australia

U.S. Department of Agriculture, A.R.S. (2013) USDA National Nutrient Database for Standard Reference, Release 26. Nutrient Data Laboratory Home Page. In

Walker, R., Browder, J., Arima, E., Simmons, C., Pereira, R., Caldas, M., Shirota, R., \& Zen, S.d. (2009) Ranching and the new global range: Amazônia in the 21st century. Geoforum, 40, 732-745

Walther, G.-R., Post, E., Convey, P., Menzel, A., Parmesan, C., Beebee, T.J., Fromentin, J.-M., Hoegh-Guldberg, O., \& Bairlein, F. (2002) Ecological responses to recent climate change. Nature, 416, 389-395

West, P.C., Gerber, J.S., Engstrom, P.M., Mueller, N.D., Brauman, K.A., Carlson, K.M., Cassidy, E.S., Johnston, M., MacDonald, G.K., Ray, D.K., \& Siebert, S. (2014) Leverage points for improving global food security and the environment. Science, 345, 325-328

White, R.P., Murray, S., Rohweder, M., Prince, S.D., \& Thompson, K.M. (2000) Grassland ecosystems. World Resources Institute Washington, DC, USA

Wilkie, D.S., Starkey, M., Abernethy, K., Effa, E.N., Telfer, P., \& Godoy, R. (2005) Role of prices and wealth in consumer demand for bushmeat in Gabon, Central Africa.

Conservation Biology, 19, 268-274

Wint, W., \& Robinson, T. (2007) Gridded livestock of the world 2007. Aportes (Costa Rica).(Jun 2004), 50-59

Young, V.R., \& Pellett, P.L. (1994) Plant proteins in relation to human protein and amino acid nutrition. The American Journal of Clinical Nutrition, 59, 1203S-1212S 


\section{CHAPTER V}

POTENTIAL GLOBAL ENERGY AND BIOFUEL YIELDS FROM CONVERTED PASTURES 


\begin{abstract}
Reducing meat consumption by humans and shifting to more efficient plant and animal protein sources could potentially free up large areas of pasture and feedcrop agriculture to produce biofuels. I examined the potential of converting pastures globally and animal feedstock agricultural lands in the U.S. and Brazil to low-input high-diversity (LIHD) biomass sources and the capacity of these systems to meet national energy demands via (1) cellulosic ethanol and (2) integrated gasification and combined cycle technology with Fischer-Tropsch hydrocarbon synthesis (IGCC-FT) processing. My analyses indicate that large amounts of energy, far in excess of many country's current demands, can potentially be produced from IGCC-FT processing of grassland biomass grown on converted pastures, especially in tropical developing countries. Over 40 countries could meet $\geq 100 \%$ of their internal demands for electricity, gasoline, and diesel. If energy products were shared between countries, the 95 countries with positive energy production yields could meet $46 \%, 28 \%$, and $39 \%$ of their combined electricity, gasoline, and diesel demands, respectively. While it is unrealistic to propose a $100 \%$ conversion of pasture lands to biofuel production, these analyses highlight the potential gains in energy production that could be achieved on already managed lands.
\end{abstract}




\section{Introduction}

Fossil fuels comprise approximately $80 \%$ of the primary energy consumed in the world, of which 58\% is consumed by the transportation sector (Nigam and Singh 2011). The projected rise of global atmospheric $\mathrm{CO}_{2}$ concentrations (Change 2007; Lawler et al. 2009) driven primarily by fossil fuel use could cause widespread climate-related stresses on natural and human systems (Rosenzweig and Parry 1994) that far exceed recent documented effects (McCarty 2001; Walther et al. 2002). Biomass-derived fuels can replace fossil fuels for many uses and thereby reduce net carbon emissions (Charles et al. 2007; Escobar et al. 2009). Indeed, some biofuel production methods are carbon negative in that they sequester more carbon through plant growth and humus production than is released through combustion and energy use associated with growing and processing (Mathews 2008; Tilman et al. 2006). Further examination and development of economical, high-efficiency and high-capacity biofuel systems is vitally important as a potentially powerful tool to help reduce net greenhouse gas emissions and consequent changes to climate.

Ethanol, derived primarily from fermentation by yeast of sugars in sugarcane and corn following hydrolysis of starch in the grain, is the leading biomass-based fuel in the world (Pimentel and Patzek 2008). Biodiesel can also be produced directly from vegetable oils of oleaginous plants such as soybean oil, rapeseed oil, and palm oil by transesterification processes or "cracking." However, it is argued that the first-generation biofuel systems that currently dominate biofuel production are not optimal to meet global needs. Problems include limited life-cycle energy efficiencies (that partly result from utilization of only a small fraction of total plant biomass); extensive use of chemical 
fertilizers and biocides; high levels of soil degradation and loss; limited to no soil carbon sequestration; low-biodiversity of monoculture production areas; and potential competition with food production (Demirbas 2008; Havlík et al. 2011).

Due to the limitations of sugar fermentation-based biofuels, research and development of other methods for creating more efficient and environmentally-friendly biofuels is expanding. Potential strategies include processing complex cellulosic components of biomass into simpler sugars prior to ethanol fermentation via chemical or enzymatic processing steps. Another strategy that may warrant additional research and development and capital investment, is the conversion of biomass into electricity, gasoline, and diesel synfuels via integrated gasification and combined cycle technology with Fischer-Tropsch hydrocarbon synthesis (IGCC-FT).

Fischer-Tropsch liquids (FTLs) are produced by first gasifying carbon-based materials under high temperature and pressure to produce $\mathrm{CO}$ and $\mathrm{H}_{2}$, which are then catalytically combined to produce straight-chained hydrocarbons that resemble semirefined crude oil. Coal and natural gas have been the primary carbon feedstocks, although biomass to liquid fuels have been achieved with changes in processing parameters designed to reduce the production of machine-gumming tars that can occur with biomass processing. FTLs can be shipped to conventional petroleum refineries for processing or refined on-site into diesel, gasoline, or jet fuel (Nigam and Singh 2011). FT fuels can be handled by existing transportation, storage, and refueling infrastructure for petroleum products, are largely compatible with current combustion engine technology, and can be blended with petroleum fuels (Takeshita and Yamaji 2008). FTLs can also be further 
distilled, hydro-cracked, cleaned and refined into a variety of raw materials for chemical industries.

An energetically-efficient and promising technology (Demirbas 2009), FT synthesis was developed in the 1930s in Germany, and is undergoing a resurgence of interest and research. Nine commercial plants utilizing fossil fuel feedstocks existed in Germany in the 1930s, one each in Texas and one in South Africa in the 1950s, and additional plants in South Africa and Malaysia in the 1990s (Dry 2002). Several plants operate today utilizing natural gas or coal as feedstocks. However, the use of biomass feedstocks is now limited to several small-scale experimental plants and commercialization is currently limited by technological challenges and especially competition with cheaper fossil fuels. Though the FT process is still a relatively expensive technology requiring large-scale production plants or further development in order to be economically viable, most oil companies have initiatives to further explore FT technology (IEA/AMF 2007).

FTLs from biomass are "high quality" in that they are free of sulfur, nitrogen, aromatics, and other contaminants typically found in petroleum products (Takeshita and Yamaji 2008). As such, the use of biomass FTLs reduces smog-inducing emissions by about $90 \%$ and emissions causing acidification and eutrophication of ecosystems by about $5-40 \%$ compared to fossil diesel or and gasoline. In addition, biomass FTLs are estimated to reduce the emissions of greenhouse gases by $60-90 \%$ vs. fossil fuels (IEA/AMF 2007). Indeed with some feedstock production systems, biomass FTLs can be carbon negative (Tilman et al. 2006). Potential sources of biomass feedstocks include agricultural by-products and dedicated feedstocks such as grasses or short-rotation trees. 
Most of these latter biofuel feedstocks are grown as monocultures that commonly require extensive application of chemical pesticides and fertilizers. However, low-input highdiversity (LIHD) mixtures of native grassland perennials have shown strong potential as a biofuel feedstock source. LIHD biomass converted via IGCC-FT can yield 51\% more usable energy per hectare of degraded infertile lands than corn-derived ethanol from fertile soils (Tilman et al. 2006). In addition, LIHD production systems have high levels of soil carbon sequestration, and have been shown to be carbon negative. For temperate grasslands ecosystem $\mathrm{CO}_{2}$ sequestration (approximately $4.4 \mathrm{Mg} / \mathrm{ha} / \mathrm{yr}$ of $\mathrm{CO}_{2}$ in soil and roots) exceeds fossil carbon dioxide releases during biofuel production (approximately $0.32 \mathrm{Mg} / \mathrm{ha} / \mathrm{yr}$ ) (Tilman et al. 2006). LIHD feedstocks also have low nutrient requirements due to increased soil nitrogen levels from the presence of legumes, require little or no pesticides or herbicides, and can provide valuable plant and wildlife habitat.

One concern with increasing the production of biofuels is the effects on food supplies and prices (Ajanovic 2011; Baka and Roland-Holst 2009; Duke et al. 2013; Escobar et al. 2009; Harvey and Pilgrim 2011; Rathmann et al. 2010; Taheripour et al. 2011; Tirado et al. 2010). This is especially true for biofuels derived from sugarcane, sugar beet, corn, and rapeseed which are staple food crops in many parts of the world. Second-generation biofuels derived from lignocellulosic sources will not compete directly with food demands, but there is still concern over competition with food on existing agriculture lands and the pressure to develop natural lands (Nigam and Singh 2011). The human population is projected to grow from 7 billion to 9 or more billion with increasing economic affluence, which will drive rising demand for more food and agricultural production, especially meat consumption (Alexandratos and Bruinsma 2012; 
Nellemann 2009). Expansion of agricultural lands is the leading cause of natural habitat destruction (Foley et al. 2005; Foley et al. 2011) and future agriculture expansion needs have been estimated to require conversion of an additional 1 billion ha of natural habitats by 2050 (Tilman et al. 2001). Given projected future food demands, further examinations of the tradeoffs between biofuel vs. food production are highly warranted.

An important factor when examining the land requirements for biofuel vs. food production is the type of food being produced. Today, livestock production is the single largest anthropogenic land use - accounting for up to $75 \%$ of all agricultural land and $30 \%$ of the Earth's land surface (Steinfeld et al. 2006a). Livestock consume $58 \%$ of human-appropriated biomass (Krausmann et al. 2008) and one-third of global cereal production (Alexandratos and Bruinsma 2012; Foley et al. 2011). However, substituting meat with soy protein as a human source of protein would reduce biomass appropriation in 2050 by $94 \%$ below 2000 baseline levels (Pelletier and Tyedmers 2010) and greatly reduce other environmental impacts related to use of water, fertilizer, fossil fuel, and biocides. (Reijnders and Soret 2003). This highlights the great potential of increasing proportional plant-based protein on a global scale to greatly reduce the area required to produce food for humans. Eliminating livestock and growing crops only for direct human consumption is estimated to increase food calories available for human consumption as much as $70 \%$, which could feed an additional 4 billion people, exceeding the projected 23 billion future people added through population growth (Cassidy et al. 2013). Changing dietary habits by reducing global per capita animal product consumption to even $10 \%$ from the current level of $20 \%$ would enable the future global population to be fed on just 
the current area of agricultural lands (Machovina and Feeley 2014b; Machovina and Feeley 2014c; McMichael et al. 2007).

Shifting to more plant-based protein sources and efficient animal protein sources could potentially free up large areas of current agricultural lands to produce biofuels. Here I investigate the potential of converting pastures and animal feedstock agricultural lands to LIHD biomass sources and the capacity of these systems to meet energy demands via cellulosic ethanol and IGCC-FT processing. The capacity of pastures to produce energy via cellulosic ethanol and IGCC-FT processing was examined on a global basis, while conversion of feedstock agricultural lands to IGCC-FT biomass sources was also assessed in the Unites States and Brazil, the two leading producers of biofuel and feedcrops. Both nations have extensive transportation demands and contrasting temperate and tropical feedstock production.

\section{Methods}

In order to estimate biomass production potential of land used to support grazing animals, I downloaded the global Pastures, v1 (2000) Map data set (Pasture Map; Fig. 5.1) and Global Patterns in Net Primary Productivity, v1 (1995) Map data set (NPP Map; Fig. 5.2) from NASA Socioeconomic Data and Applications Center (SEDAC)(Imhoff et al. 2004; Ramankutty et al. 2000) for analysis in ArcGIS 10 (ESRI 2011). The Pasture Map was created by combining agricultural inventory data with satellite data from Moderate Resolution Imaging Spectroradiometer (MODIS) and Satellite Pour 
l'Observation de la Terre (SPOT) Image Vegetation sensor. The Pasture Map provides estimates of the percent pasture cover per 0.08333 decimal degree cell (approximately $100 \mathrm{~km}^{2}$ at the equator) (Ramankutty et al. 2000). The NPP Map was created by applying the Carnegie-Ames-Stanford Approach (CASA) terrestrial carbon model (Potter et al. 1993) to global fields of normalized difference vegetation index (NDVI) from the Advanced Very High Resolution Radiometer (AVHRR) and surface climatology data from ISLSCP II (International Satellite Land Surface Climatology Project initiative II). The NPP Map provides estimates of units of elemental carbon fixed annually. It has a resolution of 0.250000 decimal degrees (approximately $784 \mathrm{~km}^{2}$ at the equator). I resampled the NPP map to 0.08333 decimal degrees $\left(\sim 100 \mathrm{~km}^{2}\right)$ and all analysis was performed at this spatial resolution.

Above ground net primary productivity (ANPP) of a cell in the NPP Map was estimated to be $50 \%$ of reported total net primary production (TNPP) based on published ANPP/TNPP ratios of 17 grassland sites (Scurlock et al. 2002). The mean value of the 17 sites was $42 \%$, but the dataset was weighted by a much higher number of temperate locations which typically allocate more NPP to subsurface tissues (Hui and Jackson 2006). The mean value of the 5 tropical sites was $58 \%$. Therefore the mean of the mean temperate and mean tropical values was chosen. For biomass production analysis, ANPP of pasture was calculated by multiplying the ANPP value (tons C) of a cell in the NPP Map by the pasture coverage value (\%) for the corresponding cell in the Pasture Map, producing a map indicating global distribution of harvestable ANPP of pasture (Pasture ANPP Map). As a comparison to the estimates of ANPP calculated in this analysis, I 
performed a linear regression of published values of ANPP from grassland study sites against my estimated ANPP values from cells containing the coordinates of the published values. Harvestable portion of production for biofuel use was estimated to be $50 \%$ of ANPP, with remainder of annual aboveground production left for natural ecosystem processes, soil building, and carbon sequestration. Total ANPP was summed for each country that contained pastures.

The total areas of production of livestock feedcrop corn, biofuel corn, feedcrop soy, feedcrop wheat, and biofuel sugarcane were estimated during 2012 for the U.S. and Brazil from literature and data sources (Advisor 2014; Association 2014; Birt 2012; USDA 2014). For analysis of dry weight biomass production of LIHD grasslands replacing croplands, a value of $6,000 \mathrm{~kg} / \mathrm{ha}$ was used for the U.S. based on previous estimates for fertile cropland soils (Tilman et al. 2006). For Brazil, a conservative value of $20,000 \mathrm{~kg} / \mathrm{ha}$ was used based on previous estimates for grazed unfertilized mixed grass/legume tropical pasture (Ibrahim and 'T Mannetje 1998). Harvestable biomass for energy production was estimated at $50 \%$ of ANPP.

Ethanol production via cellulosic methods was estimated at a rate of $0.255 \mathrm{~L} / \mathrm{kg}$ dry weight of biomass, with an additional $9.2 \%$ of ethanol energy yield also produced via simultaneous electrical production. Dry weight biomass of production from the ANPP Pasture Map was estimated to be $2 \mathrm{X}$ the amount of carbon. IGCC-FT processing energy yields are estimated as $48.75 \%$ higher than cellulosic ethanol, and products are divided into electricity (47\%) and liquid fuels (53\%), the latter further divided into diesel (62\%) and gasoline (38\%) similar to the methodology of Tilman et al. (Tilman et al. 2006). 
Energy required for biomass production, harvesting, and transport (4 GJ/ha) were deducted to estimate net energy produced by both cellulosic ethanol and IGCC-FT methods (Tilman et al. 2006). Total IGCC-FT electrical, diesel, and gasoline production potential for countries with pasture were compared to within-country demand for the same conventionally produced products in 2012 (Administration 2012) and a net energy balance for each country was calculated for electricity, diesel, and gasoline.

For all analyses only cells containing $\geq 25 \%$ coverage by pasture in the Pasture Map were included. In addition, I excluded all protected areas from analysis (protected areas were identified and mapped using data downloaded from the World Database on Protected Areas; www.wdpa.org).

\section{Results}

The ANPP Pasture Map (Fig. 5.3) indicates higher productivity rates in tropical areas, although temperate zones contain large extents of pasture. Harvestable pastures are found in 104 countries. ANPP of pastures ranges from 37.6 to $118,455.7$ tons $\mathrm{C} / 100 \mathrm{~km}^{2}$ with a mean ANPP of 165.3 tons $\mathrm{C} / 100 \mathrm{~km}^{2}$. ANPP values from literature were compiled from 146 geographic locations (Fig. 5.3b) that corresponded to equivalent locations on the ANPP Pasture Map. The ANPP values from literature are significantly higher than the ANPP estimates from the Pasture Map at the same locations (t-test $\left.\mathrm{p}=4.9 \times 10^{-7}\right)$. Across all 146 sample locations, the mean ANPP from the Pasture Map was 6,477 tons $\mathrm{C} / 100 \mathrm{~km}^{2}, 42 \%$ less than the mean value of ANPP values reported from the literature $\left(11,125\right.$ tons $\left.\mathrm{C} / 100 \mathrm{~km}^{2}\right)$. ANPP estimates form the literature are higher than estimates 
form the pasture map in 104 of the of the 146 sample locations ( $71 \%$ of the sites; Fig. $5.4)$.

The biomass yields of tropical countries are much higher, with Uruguay leading all the countries with a mean yield of 573 tons $/ \mathrm{km}^{2}$ and Saudi Arabia having the lowest yield at 13 tons $/ \mathrm{km}^{2}$. The mean yield of the 104 countries is 196 tons $/ \mathrm{km}^{2}$ (Tables $5.1 \&$ 5.2). If adjusted to correct for the mean $42 \%$ lower ANPP values calculated in the Pasture Map than reported for similar locations from the literature, the highest yields would be 986 tons $/ \mathrm{km}^{2}$ and the mean yield of the 104 countries would be 337 tons $/ \mathrm{km}^{2}$.

Total estimated harvestable biomass from pastures ( $50 \%$ of ANPP) varies widely by country from a low of $\sim 36,000$ dry tons of biomass in Saudi Arabia to a high of $\sim 1.35$ billion tons in Brazil (Table 5.1). The total harvestable biomass worldwide is $\sim 7.7$ billion tons. The leading 25 countries contain $\sim 85 \%$ ( $\sim 6.5$ billion tons) of this production (Table 5.2), and the leading 5 countries contain $\sim 43 \%$ ( $\sim 3.3$ billion tons). The amount of harvestable biomass is not a simple function of area since there are large expanses of pasture in temperate zones that have lower harvestable biomass than smaller tropical areas. For example, Kazakhstan, which is ranked $3^{\text {rd }}$ in harvestable area is ranked $13^{\text {th }}$ in harvestable biomass, and Angola, which is ranked $13^{\text {th }}$ in harvestable area is ranked $5^{\text {th }}$ in harvestable biomass.

The conversion of biomass to cellulosic ethanol (accounting for energy required to harvest, transport and process biomass) can potentially yield high amounts of ethanol in many countries (Table 5.1), with Brazil having the potential to produce the largest amount at $\sim 315$ billion liters. Fifteen countries, all in temperate and desert climates, are 
estimated to produce net negative amounts of ethanol energy (i.e., requiring more energy to harvest, transport and process than is produced). The total amount of ethanol that can potentially be produced from the 89 positive yielding countries is $\sim 1.3$ trillion liters.

The conversion of biomass to the three simultaneously FT-derived energy products - electricity, gasoline, and diesel (accounting for energy required to harvest, transport and process biomass) - can potentially yield high levels of output in many countries (Table 5.1; Fig 5.5). Brazil is the largest potential producer with an estimated potential output of $\sim 1.4$ billion $\mathrm{MwH}$ of electricity, $\sim 61$ billion liters of gasoline, and $\sim 89$ billion liters of diesel. Nine temperate and desert climate countries will be unable to produce net positive amounts of electricity, gasoline, and diesel (i.e., requiring more energy to harvest, transport and process than is produced). The total electricity, gasoline, and diesel that can be produced from the 95 positive yielding countries is estimated as $\sim 6.5$ billion MwH, 289 billion liters, and 424 billion liters, respectively.

Using the biomass produced on pastures, fifty-five countries could potentially produce enough electricity to meet at least $100 \%$ of their current internal demand, while 43 countries could produce more than twice their demand, and 22 countries could produce more than 10 times their demand (Fig. 5.6a). Brazil, the largest biomass producer, could produce 3 times its current internal electricity demands. However, the United States, ranked $4^{\text {th }}$ in amount of harvestable biomass, could meet only $10 \%$ of its current internal electricity demand. In terms of gasoline, forty-one countries could produce enough gasoline through biomass conversion to meet at least $100 \%$ of their current internal demand, while 31 countries could produce more than twice their demand, 
and 15 countries could produce more than 10 times their demand (Fig. 5.6b). Brazil could meet 2.7 times it internal gasoline demand, but the U.S. could supply less than $4 \%$ of its internal demand. In terms of diesel, forty-three countries could produce enough diesel to meet at least $100 \%$ of their current internal demand, while 28 countries could produce more than twice their demand, and 13 countries could produce more than 10 times their demand (Fig. 5.6c). Brazil could meet 1.8 times its internal diesel demand, but the U.S. could supply only $12 \%$ of its internal demand. If energy products were shared between the 95 countries with positive energy production yields, $46 \%, 28 \%$, and $39 \%$ of the 95 countries' combined electricity, gasoline, and diesel demands could be met, respectively. If the FT energy available from these 95 countries was used to address total global demand, $35 \%, 23 \%$, and $29 \%$ of electricity, gasoline, and diesel demands could be met.

The total amount of FT electricity, gasoline and diesel that could be produced on current feedcrop and biofuel croplands of the U.S. and Brazil indicate that Brazil has a much greater potential for producing fuels, meeting national energy demands, and even exporting energy from these lands (Table 5.3). The United States used approximately 3.9 billion MwH of electricity, 521.8 billion liters of gasoline, and 220.5 billion liters of diesel in 2012, whereas Brazil's demand was only 0.5 billion MwH, 22.8 billion liters, and 38.2 billion liters of electricity, gasoline, and diesel, respectively. The U.S.A. could supply $5 \%, 2 \%$, and $6 \%$ of its internal demand for electricity, gasoline, and diesel, respectively from lands used for feedcrop production (corn, soy, and wheat), Brazil could meet $97 \%, 86 \%, 58 \%$ of its internal demand for electricity, gasoline, and diesel, respectively from lands used for feedcrop production (corn and soy). When current areas 
used for ethanol production and pasture areas are added to the feedcrop land production potential, the U.S. could supply $18 \%, 7 \%$, and $21 \%$ of its internal demand for electricity, gasoline, and diesel, respectively, and Brazil could supply $421 \%, 373 \%$, and $252 \%$ of its internal demand for electricity, gasoline, and diesel, respectively.

\section{Discussion}

Based on ANPP estimates from the map analysis, large amounts of energy, far in excess of many country's current internal demands, can potentially be produced if current pasture lands were converted to biofuel production. Many countries have large areas of pasture and/or high enough productivity levels that pasture biomass could be a potentially useful and viable energy source. The 25 countries with the largest areas of pasture contain a combined $\sim 36$ million $\mathrm{km}^{2}$ of pasture, an area $\sim 3.7$ times the size of the U.S.A., producing $\sim 6.5$ billion tons of biomass. Tropical countries with large areas of pasture are especially suitable for energy production as biomass yields are much higher (i.e. $\sim 315$ tons $/ \mathrm{km}^{2}$ in Brazil vs. $\sim 86$ tons $/ \mathrm{km}^{2}$ in the U.S.A.). The 25 countries with the highest mean harvestable biomass yields (combined accounting for a $50 \%$ of total ANPP) are all located in the tropics.

Estimates of ANPP via the map analysis in this study may have underestimated true ANPP. Literature-reported values were an average of $72 \%$ higher than the mean value of the same locations calculated via the map analysis. As another comparison, published ANPP values from 24 locations of pasture across climate gradients in Argentina (Irisarri et al. 2014) ranged from 6,888 to 52,011 tons $\mathrm{C} / 100 \mathrm{~km}^{2}$ (mean $=26,908$ tons $\mathrm{C} / \mathrm{km}^{2}$ ), which was $81 \%$ higher the mean ANPP value from the map 
analysis for Argentina $\left(14,892\right.$ tons $\left.\mathrm{C} / 100 \mathrm{~km}^{2}\right)$. The lower estimates in the map analysis may be due estimates of NPP reported in the NPP Map data set that result from the coarse resolution ( $784 \mathrm{~km}^{2}$ at the equator) and the inclusion of non-pasture areas in grid cells. Since my analyses used the map-derived estimates of yield, my estimates of biomass productivity levels and the estimates of potential energy product yields are conservative.

Pastures with potentially net positive energy production via cellulosic ethanol are found in 89 countries. Production capacity is high in many countries, especially in the tropics. Brazil, the country with the greatest potential cellulosic ethanol production, could produce an estimated $\sim 315$ billion $\mathrm{L}$ from $3,239,700 \mathrm{~km}^{2}$ of pasture-grown biomass. This is nearly 4 times the production of China which has the next highest potential ( 72 billion L). In 2011-12, Brazil produced 22.7 billion L of ethanol from 559.2 million tons (fresh weight) of sugarcane harvested from 97,000 $\mathrm{km}^{2}$ (Association 2015). This amount of fresh sugar cane production equals approximately 84 million tons when converted (15\% conversion rate) to dry matter (Mendoza et al. 1980). Brazil's total sugarcane ethanol production is approximately $7.2 \%$ of the production volume via cellulosic ethanol from pastures estimated from 1.35 billion tons of dry matter biomass in this study, but the sugarcane harvest area is only $3 \%$ of the area of pastures. Therefore, current ethanol production methods from sugarcane have over twice the yields $(\sim 234,000 \mathrm{~L} / \mathrm{km} 2)$ of those estimated for cellulosic ethanol from pastures in this study $(97,000 \mathrm{~L} / \mathrm{km} 2)$. If the dry weight production of sugarcane was processed into ethanol via cellulosic methods $(0.255 \mathrm{~L} / \mathrm{kg})$, the output would equal $\sim 21.4$ billion L, approximately the same as the reported 22.7 billion $\mathrm{L}$ production levels for current ethanol production. The straw and 
dry and green leaves of sugarcane biomass ( $\sim 25 \%$ of ANPP) is burned in the fields, and its inclusion into cellulosic ethanol production would increase outputs. The mean dry weight yield of sugarcane produced in Brazil in 2012 was 864 tons $/ \mathrm{km}^{2}$, which is twice the mean yield for pastures in Brazil calculated in this study via the map analysis (417 tons $/ \mathrm{km}^{2}$ ). Sugarcane, which requires large amounts of fossil-fuel based fertilizer applications (Keating et al. 1997), has among the highest productivity rates of any plant. A key difference in the ethanol yields calculated in this study is the use of only $50 \%$ of ANPP for ethanol production. As mentioned above a larger percentage of sugarcane ANPP is harvested for production ( $>75 \%)$ following pre-harvest burning of green and dry leaves. Accounting for this difference would make yields of sugarcane ethanol and pasture cellulosic ethanol similar. Also, as mentioned above, pasture yields calculated via map analysis in this study may be underestimating actual production potentials. Tropical pasture consisting of mixed grass/legume species have been reported to yield $\sim 2000$ tons/ $\mathrm{km}^{2}$ dry weight (Ibrahim and 'T Mannetje 1998).

The potential production levels of electricity, gasoline, and diesel via IGCC-FT processing from pasture is high for many countries. Over 40 countries could potentially meet their current internal demands for electricity, gasoline, and diesel using pasturederived biomass. The production to demand ratios for these products is especially high in developing tropical countries. Production potential can exceed internal demands by more than 10 fold in many underdeveloped African nations, such as Chad, Somalia, Madagascar, Guinea-Bissau, Angola, Tanzania, Central African Republic, and Mali. Several less developed countries of South America, such as Bolivia and Paraguay, also 
have high production potential to demand ratios. Several more developed countries with large areas of pasture in subtropical or tropical climates, including Argentina, Colombia, and Australia, can potentially meet or exceed current internal energy demands. However, in most temperate countries, the lower ANPP combined with high energy demands creates a scenario where only small portions of national electricity and fuel demands can be met despite large expanses of harvestable pasture. For example, Russia has the $6^{\text {th }}$ largest area of harvestable pastures $\left(\sim 2\right.$ million $\left.\mathrm{km}^{2}\right)$ but can meet only $5 \%, 4 \%$, and $8 \%$ of its internal demand for electricity, gasoline, and diesel, respectively.

The difference in the abilities of tropical vs. temperate countries to produce energy from pasture biomass is clear when examining the differences between Brazil and the U.S. for existing pastures, as well as for potential pastures gained from converting existing feedcrop agricultural areas to biomass-producing pastures. Both countries have large areas of existing pasture with Brazil having 1.3 times more harvestable area than the U.S.A. However, the harvestable biomass for Brazil is 3.2 times greater primarily because the mean yield is over twice as high. Brazil also has much lower demand for energy products with levels of consumption of electricity, gasoline, and diesel $12 \%, 4 \%$, and $22 \%$, respectively, of the levels of consumption in the U.S. Because of this lower demand and higher yields, Brazil could meet $303 \%, 268 \%$, and $181 \%$ while the U.S. could meet only $11 \%, 4 \%$, and $12 \%$ of their internal electricity, gasoline, and diesel demands, respectively, from existing pastures.

The U.S.A. has 1.6 times the area of land dedicated to growing livestock feedcrops $\left(391,857 \mathrm{~km}^{2}\right.$ for corn, soy, and wheat) compared to Brazil $\left(238,582 \mathrm{~km}^{2}\right.$ for 
corn and soy). The yields used in this study for estimating potential production of grasslands established by converting fertile croplands are over 3 times higher for tropical Brazil than temperate U.S. soils. Because of these higher tropical yields and lower demand for energy products, Brazil could meet $97 \%, 86 \%$, and $58 \%$ of its demand for electricity, gasoline, and diesel, respectively by converting feedcrop lands to grasslands for FT-biomass production, whereas the U.S. could meet only $5 \%, 2 \%$, and $6 \%$ of its demand for electricity, gasoline, and diesel, respectively. Brazil does have much higher use of ethanol for transportation, and if this fuel demand was instead converted to gasoline, Brazil could still meet over $40 \%$ of its internal gasoline demands. If the current area dedicated to sugarcane ethanol production and pasture are added to the potential production of feedcrop land, Brazil could produce 4.2, 3.7 and 2.5 times its internal demand for electricity, gasoline, and diesel, respectively, making Brazil a potential exporter of these energy products. However, combining feedcrop, corn ethanol, and pasture lands could only supply $18 \%, 7 \%$, and $21 \%$ of the U.S. internal demand for electricity, gasoline, and diesel, respectively.

The results of this study indicate that there is great potential to meet energy needs of many countries from IGCC-FT processing of grassland biomass, especially in the tropics. The energy returns for IGCC-FT processing of LIHD grassland biomass are much greater than current ethanol production methods. For example, LIHD biomass converted via IGCC-FT in the temperate U.S. has been estimated by Tilman et al. (Tilman et al. 2006) to yield 51\% more usable energy per hectare from degraded infertile land than does corn grain ethanol from fertile soils $\left(28.4 \mathrm{GJ} \mathrm{ha}^{-1}\right.$ vs. $\left.18.8 \mathrm{GJ} \mathrm{ha}^{-1}\right)$, and 
fertile lands can yield about 50\% more LIHD biomass (and bioenergy) than degraded lands (Tilman et al. 2006). The same study estimated energy inputs of $4.01 \mathrm{GJ} \mathrm{ha}^{-1} \mathrm{yr}^{-1}$ for a biomass yield of 3,682 $\mathrm{kg} \mathrm{ha}^{-1} \mathrm{yr}^{-1}$ on degraded temperate soils, and $4.64 \mathrm{GJ} \mathrm{ha}^{-1} \mathrm{yr}^{-1}$ to produce $6,000 \mathrm{~kg} \mathrm{ha}^{-1} \mathrm{yr}^{-1}$ of LIHD biomass on fertile soils. If I use the latter higher input energy requirements for tropical grasslands, which can produce $20,000 \mathrm{~kg} \mathrm{ha}^{-1} \mathrm{yr}^{-1}$ of biomass, then net energy produced via IGCC-FT processing of tropical LIHD grasslands could yield net energy of $171 \mathrm{GJ} \mathrm{ha}^{-1} \mathrm{yr}^{-1}$ if $100 \%$ of ANPP is harvested. Harvesting only $50 \%$ of ANPP would still yield $85 \mathrm{GJ} \mathrm{ha}^{-1} \mathrm{yr}^{-1}$. Sugarcane ethanol net energy yields are approximately $113 \mathrm{GJ} \mathrm{ha}^{-1} \mathrm{yr}^{-1}$ (De Oliveira et al. 2005), which is produced via a monoculture system that burns and harvests nearly all ANPP and which requires large amounts of nutrient inputs.

In addition to the higher energy yields achievable through IGCC-FT processing of LIHD grasslands, converting pasture and croplands to LIHD grasslands that retain 50\% of ANPP would protect and build soils, sequester carbon, and improve biodiversity. Grasslands and their extensive shoot and fibrous root systems, except when overgrazed, provide protective cover for soils and prevent wind and water erosion. The death of roots and shoots incorporates carbon into soils, and grasslands hold large reservoirs of carbon that grow over time. Tilman et. al. (Tilman et al. 2006) estimated that temperate LIHD grasslands have net ecosystem carbon dioxide sequestration (4.4 megagram hectare ${ }^{-1}$ year $^{-1}$ of carbon dioxide in soil and roots) that greatly exceeds fossil carbon dioxide released during IGCC-FT biofuel production (0.32 megagram hectare ${ }^{-1}$ year $\left.^{-1}\right)$. Grasslands are also important reservoirs of biodiversity. A recent study which assembled 
the maximum values recorded for vascular plant species richness for contiguous areas from $1 \mathrm{~mm}^{2}$ up to 1 ha concluded that only two community types contain global plant species maxima. The maxima at large spatial scales were tropical rain forests, but the maxima at smaller spatial grain were from oligo- to meso-trophic, managed, semi-natural, temperate grasslands (e.g. 89 species per $\mathrm{m}^{2}$ ) (Wilson et al. 2012).

Actual amounts of cellulosic ethanol and IGCC-FT -derived electricity, gasoline, and diesel production capacities from pastures could potentially be $\sim 70 \%$ higher than estimated in this study via the map analysis. Therefore, fuel production capacities presented from this research are likely very conservative estimates, yet even these amounts could meet or make significant contributions to internal energy demands for many countries. However, several technological and economic challenges face the development of this energy source including (1) limited biomass based IGCC-FT plant development, (2) competition from less expensive fossil fuels, (3) extensive biomass transportation infrastructure needs, (4) competition with livestock demands, and (5) higher potential yields from algal based biofuels.

Currently only about a dozen biomass based IGCC-FT plants exist, and none are utilizing grassland biomass sources. Most existing plants are pilot or smaller demonstration-type facilities. However, one company, Cool Planet Energy Systems, broke ground on a plant in 2014 in Louisiana that will annually produce $\sim 38$ million L of gasoline from pine chips. British Airways plans to produce 50 million $\mathrm{L}$ of jet fuel from organic waste. A recent study (Hannula and Kurkela 2013) evaluated 20 individual large-scale biomass-to-liquid plant designs based on their technical and economic 
performance. The analysis showed that it is possible to produce sustainable low-carbon fuels from lignocellulosic biomass with first-law efficiency in the range of 49.6-66.7\% depending on the end-product and process conditions, and that sustainable low carbon fuels could be produced on parity with $\$ 110$ to $\$ 150$ per barrel crude oil price. The lower end of these production cost estimates are close to long-term price forecasts for crude oil and may not need substantial incentives to break even. However, new plants are likely to be smaller and projected to have much higher costs and subsidy requirements.

An important challenge to developing biomass based supplies is the large transportation network required to gather and ship biomass to IGCC-FT plants. A recent study (IEA/AMF 2007) estimated that to substitute $15 \%$ of the EU 15 countries fuel consumption would require $122 \mathrm{FT}$-plants of $1.6 \mathrm{GW}$ utilizing fast-growing willow trees (Salix sp.) as a biomass source covering an area the size of Poland. This would require a large number of trucks to ship biomass. For a modeled plant in Poland, this would be equivalent to 5 times as many trucks visiting the plant as visit the largest paper plants in the Nordic countries. Any biomass based energy source will require large storage and internal logistic facilities, including establishment of railways.

Perhaps the main limiting factor facing the conversion of pastures to biofuel production is the demand for the ruminant livestock products - beef, lamb, and goat that are now being raised on pastures. The potential energy source of pastures could only be utilized if global consumption of these meat products decreases. There were a reported 3.6 billion domestic ruminants on Earth in 2011 (1.4 billon cattle, 1.1 billion sheep, 0.9 billion goats and 0.2 billon buffalo), and on average, 25 million domestic ruminants have 
been added to the planet each year over the past 50 years (Ripple et al. 2014b). Between 2000 and 2050, the global cattle population may increase from 1.5 billion to 2.6 billion, and the global goat and sheep population from 1.7 billion to 2.7 billion, increases of $73 \%$ and 59\%, respectively (Hubert et al. 2010). Although red meat consumption is decreasing in many developed countries, developing countries are increasing consumption. Meat products can also be produced more intensively by using crop-based feeds, requiring less land and potentially freeing up some pastures for biomass-based fuel production. Reducing consumption of ruminants by humans and replacing it with plantbased protein or more efficient land-use animal protein sources (Machovina and Feeley 2014a) would be a critical step in utilizing grassland biomass for fuel production.

Although fuel production capacities via pasture-based IGCC-FT plants are large for many countries, more efficient renewable-based fuels that require less land area may be preferential for research, investment, and development. For example, cyanobacterial platform organisms engineered to directly produce alkanes from carbon sources (i.e. $\mathrm{CO}_{2}$ ) have great potential to efficiently produce fuel from small areas of ponds (Robertson et al. 2011; Schirmer et al. 2010). Photosynthetic microorganisms have higher growth rates than terrestrial plants, and the production systems can be based on nonarable land (Machado and Atsumi 2012). However, more research into potentially utilizing grasslands for biofuel production is warranted given their potential to make significant contributions to global energy supply.

Shifting meat consumption away from ruminants (cattle, goats, buffalo), which are the primary users of grasslands, to monogastrics (pigs and chickens) and supplying 
feedstocks from intensive cropland agriculture or better yet, integrated mixed-crop systems, could reduce livestock land use as well as methane production, a potent greenhouse gas. Reducing meat consumption to $10 \%$ of global diets (a $50 \%$ reduction with most reduction occurring in developed countries), as well as shifting to more efficient plant-based or animal-based protein sources, would reduce both $\mathrm{CO} 2$ and methane production and free up large areas of current agricultural lands to produce biofuels. While it is unrealistic to propose a $100 \%$ conversion of pasture lands or feedcrop agricultural lands to biofuel production, these analyses highlight the potential gains in energy production that could be achieved on already managed lands with a reduction in animal product consumption by humans. When combined with increased energy-use efficiencies by consumers, LIHD IGCC-FT biomass could potentially be an important, carbon negative energy source that helps address global climate change. 


\begin{tabular}{|c|c|c|c|c|c|c|c|c|c|c|}
\hline Country & $\begin{array}{c}\text { Harvestable } \\
\text { Area }\left(\mathbf{k m}^{2}\right)\end{array}$ & $\begin{array}{c}\text { Harvestable } \\
\text { Biomass } \\
\text { (million } \\
\text { tons) }\end{array}$ & $\begin{array}{c}\text { Mean } \\
\text { Yield } \\
\left(\text { tons } / \mathbf{k m}^{2}\right)\end{array}$ & $\begin{array}{c}\text { Ethanol } \\
\text { (billion } \\
\text { L) }\end{array}$ & $\begin{array}{c}\text { Electricity } \\
\text { (million } \\
\text { MWh) }\end{array}$ & $\begin{array}{c}\text { Gasoline } \\
\text { (billion } \\
\text { L) }\end{array}$ & $\begin{array}{c}\text { Diesel } \\
\text { (billion } \\
\text { L) }\end{array}$ & $\begin{array}{c}\text { Electricity } \\
\text { Prod/Use }\end{array}$ & $\begin{array}{l}\text { Gasoline } \\
\text { Prod/Use }\end{array}$ & $\begin{array}{c}\text { Diesel } \\
\text { Prod/Use }\end{array}$ \\
\hline Brazil & $3,239,700$ & $1,349.48$ & 417 & 314.65 & $1,379.01$ & 61.07 & 89.60 & 3.03 & 2.68 & 1.81 \\
\hline China & $4,871,500$ & 588.14 & 121 & 71.86 & 420.20 & 18.61 & 27.30 & 0.12 & 0.20 & 0.16 \\
\hline Australia & $4,349,500$ & 545.86 & 125 & 69.93 & 398.98 & 17.67 & 25.92 & 1.87 & 0.94 & 1.33 \\
\hline United States & $2,498,600$ & 477.69 & 191 & 85.87 & 417.49 & 18.49 & 27.13 & 0.11 & 0.04 & 0.12 \\
\hline Angola & 816,000 & 326.63 & 400 & 75.56 & 332.11 & 14.71 & 21.58 & 72.33 & 10.58 & 12.03 \\
\hline Sudan & $1,575,300$ & 290.17 & 184 & 51.08 & 250.60 & 11.10 & 16.28 & 39.41 & 7.27 & 4.86 \\
\hline Argentina & $1,875,400$ & 279.29 & 149 & 42.38 & 222.42 & 9.85 & 14.45 & 2.00 & 1.78 & 1.07 \\
\hline Mozambique & 552,800 & 251.20 & 454 & 59.52 & 259.32 & 11.48 & 16.85 & 25.39 & 54.63 & 28.41 \\
\hline Colombia & 570,200 & 237.64 & 417 & 55.42 & 242.86 & 10.75 & 15.78 & 5.36 & 2.96 & 2.52 \\
\hline Bolivia & 520,000 & 218.79 & 421 & 51.11 & 223.85 & 9.91 & 14.54 & 38.42 & 9.49 & 10.74 \\
\hline Tanzania & 489,400 & 204.69 & 418 & 47.76 & 209.27 & 9.27 & 13.60 & 61.50 & 27.89 & 15.56 \\
\hline South Africa & $1,242,500$ & 201.98 & 163 & 32.80 & 166.80 & 7.39 & 10.84 & 0.78 & 0.63 & 1.13 \\
\hline Kazakhstan & $3,821,000$ & 190.27 & 50 & -19.11 & 18.60 & 0.82 & 1.21 & 0.26 & 0.18 & 0.35 \\
\hline Paraguay & 345,100 & 182.97 & 530 & 44.44 & 191.90 & 8.50 & 12.47 & 28.31 & 20.08 & 11.20 \\
\hline Mexico & $1,084,500$ & 151.27 & 139 & 21.66 & 116.88 & 5.18 & 7.59 & 0.55 & 0.11 & 0.31 \\
\hline Zambia & 356,200 & 135.74 & 381 & 31.08 & 137.13 & 6.07 & 8.91 & 17.23 & 28.52 & 27.21 \\
\hline Uruguay & 233,400 & 133.76 & 573 & 32.84 & 141.27 & 6.26 & 9.18 & 15.50 & 11.68 & 7.35 \\
\hline Russian Fed. & $2,000,600$ & 128.58 & 64 & -1.94 & 42.97 & 1.90 & 2.79 & 0.05 & 0.04 & 0.08 \\
\hline Madagascar & 369,800 & 122.97 & 333 & 27.26 & 121.76 & 5.39 & 7.91 & 108.11 & 51.81 & 19.97 \\
\hline Mongolia & $1,592,100$ & 120.20 & 75 & 3.43 & 54.71 & 2.42 & 3.55 & 13.85 & 6.27 & 6.01 \\
\hline Venezuela & 274,200 & 97.90 & 357 & 22.09 & 98.00 & 4.34 & 6.37 & 1.07 & 0.27 & 0.66 \\
\hline Chad & 570,000 & 94.60 & 166 & 15.59 & 78.75 & 3.49 & 5.12 & 864.06 & 285.73 & 95.87 \\
\hline Dem.. Congo & 224,500 & 72.16 & 321 & 15.86 & 71.06 & 3.15 & 4.62 & 11.47 & 11.32 & 16.43 \\
\hline
\end{tabular}




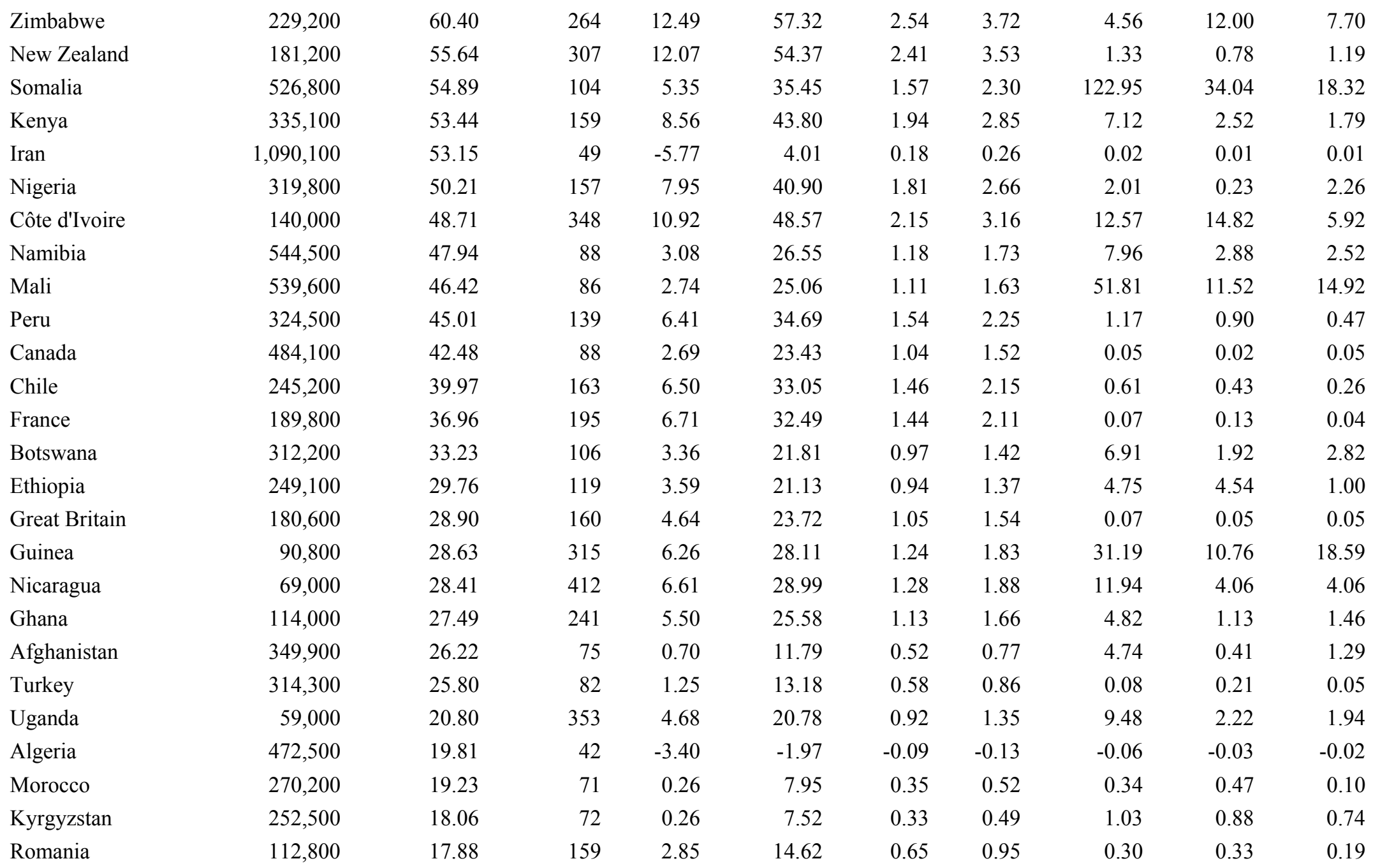




\begin{tabular}{|c|c|c|c|c|c|c|c|c|c|}
\hline Ireland & 112,200 & 17.71 & 158 & 2.81 & 14.46 & 0.64 & 0.94 & 0.57 & 0.33 \\
\hline Ecuador & 86,600 & 17.64 & 204 & 3.28 & 15.71 & 0.70 & 1.02 & 1.07 & 0.22 \\
\hline Domin. Rep. & 38,900 & 16.15 & 415 & 3.76 & 16.50 & 0.73 & 1.07 & 1.26 & 0.56 \\
\hline Uzbekistan & 395,100 & 15.82 & 40 & -3.05 & -2.49 & -0.11 & -0.16 & -0.06 & -0.06 \\
\hline Niger & 353,100 & 15.27 & 43 & -2.41 & -0.93 & -0.04 & -0.06 & -1.12 & -0.42 \\
\hline Guatemala & 47,600 & 15.12 & 318 & 3.31 & 14.86 & 0.66 & 0.97 & 1.87 & 0.51 \\
\hline Turkmenistan & 448,700 & 14.99 & 33 & -4.29 & -6.26 & -0.28 & -0.41 & -0.56 & -0.26 \\
\hline Spain & 116,500 & 14.55 & 125 & 1.85 & 10.60 & 0.47 & 0.69 & 0.04 & 0.06 \\
\hline Cuba & 44,600 & 14.29 & 320 & 3.14 & 14.07 & 0.62 & 0.91 & 1.03 & 1.73 \\
\hline Italy & 53,900 & 11.93 & 221 & 2.31 & 10.88 & 0.48 & 0.71 & 0.04 & 0.03 \\
\hline Costa Rica & 33,100 & 11.42 & 345 & 2.56 & 11.38 & 0.50 & 0.74 & 1.33 & 0.51 \\
\hline Senegal & 85,300 & 9.15 & 107 & 0.94 & 6.04 & 0.27 & 0.39 & 2.72 & 1.73 \\
\hline Cent. Afr. R. & 33,000 & 8.97 & 272 & 1.87 & 8.56 & 0.38 & 0.56 & 57.55 & 12.71 \\
\hline Burkina Faso & 93,600 & 8.69 & 93 & 0.65 & 5.07 & 0.22 & 0.33 & 6.56 & 1.18 \\
\hline Germany & 56,800 & 7.76 & 137 & 1.09 & 5.94 & 0.26 & 0.39 & 0.01 & 0.01 \\
\hline Malawi & 24,200 & 7.43 & 307 & 1.61 & 7.26 & 0.32 & 0.47 & 3.96 & 3.04 \\
\hline Swaziland & 19,000 & 7.41 & 390 & 1.70 & 7.51 & 0.33 & 0.49 & 7.09 & 2.78 \\
\hline Georgia & 39,400 & 7.23 & 184 & 1.27 & 6.24 & 0.28 & 0.41 & 0.82 & 0.56 \\
\hline Congo & 28,800 & 6.86 & 238 & 1.37 & 6.36 & 0.28 & 0.41 & 11.04 & 1.46 \\
\hline India & 59,500 & 6.51 & 109 & 0.69 & 4.35 & 0.19 & 0.28 & 0.01 & 0.01 \\
\hline Lesotho & 30,400 & 6.42 & 211 & 1.21 & 5.78 & 0.26 & 0.38 & 18.82 & 2.65 \\
\hline Austria & 38,200 & 5.99 & 157 & 0.95 & 4.88 & 0.22 & 0.32 & 0.08 & 0.09 \\
\hline Eritrea & 96,700 & 5.73 & 59 & -0.23 & 1.52 & 0.07 & 0.10 & 6.00 & 5.51 \\
\hline Azerbaijan & 54,100 & 5.71 & 106 & 0.57 & 3.72 & 0.16 & 0.24 & 0.27 & 0.12 \\
\hline Panama & 25,100 & 5.16 & 206 & 0.96 & 4.61 & 0.20 & 0.30 & 0.74 & 0.33 \\
\hline Guinea-Bissau & 18,700 & 5.13 & 274 & 1.07 & 4.90 & 0.22 & 0.32 & 78.70 & 7.79 \\
\hline
\end{tabular}




\begin{tabular}{|c|c|c|c|c|c|c|c|c|c|c|}
\hline El Salvador & 14,900 & 5.05 & 339 & 1.13 & 5.02 & 0.22 & 0.33 & 0.93 & 0.38 & 0.45 \\
\hline Netherlands & 28,500 & 4.94 & 173 & 0.84 & 4.18 & 0.18 & 0.27 & 0.04 & 0.03 & 0.02 \\
\hline Croatia & 32,600 & 4.87 & 149 & 0.74 & 3.89 & 0.17 & 0.25 & 0.25 & 0.20 & 0.12 \\
\hline Cameroon & 29,700 & 4.54 & 153 & 0.70 & 3.66 & 0.16 & 0.24 & 0.71 & 0.35 & 0.29 \\
\hline Bulg. & 30,200 & 4.22 & 140 & 0.60 & 3.26 & 0.14 & 0.21 & 0.11 & 0.18 & 0.10 \\
\hline Serb. Mont. & 27,000 & 3.81 & 141 & 0.55 & 2.97 & 0.13 & 0.19 & 0.10 & 0.20 & 0.11 \\
\hline Tajikistan & 66,300 & 3.60 & 54 & -0.25 & 0.66 & 0.03 & 0.04 & 0.05 & 0.25 & 0.16 \\
\hline Indonesia & 17,400 & 3.48 & 200 & 0.64 & 3.08 & 0.14 & 0.20 & 0.02 & 0.01 & 0.01 \\
\hline Burundi & 11,300 & 3.38 & 299 & 0.73 & 3.29 & 0.15 & 0.21 & 14.85 & 4.67 & 6.43 \\
\hline Rwanda & 8,200 & 2.93 & 357 & 0.66 & 2.93 & 0.13 & 0.19 & 9.17 & 1.81 & 1.87 \\
\hline Gabon & 8,700 & 2.65 & 304 & 0.57 & 2.58 & 0.11 & 0.17 & 1.79 & 1.48 & 0.27 \\
\hline Armenia & 21,500 & 2.58 & 120 & 0.31 & 1.83 & 0.08 & 0.12 & 0.39 & 0.08 & 0.13 \\
\hline Slovakia & 18,700 & 2.35 & 126 & 0.30 & 1.72 & 0.08 & 0.11 & 0.07 & 0.09 & 0.06 \\
\hline Tunisia & 45,500 & 2.33 & 51 & -0.21 & 0.29 & 0.01 & 0.02 & 0.02 & 0.02 & 0.01 \\
\hline Cambodia & 10,600 & 2.25 & 213 & 0.43 & 2.03 & 0.09 & 0.13 & 1.01 & 0.42 & 0.20 \\
\hline Pakistan & 56,000 & 2.09 & 37 & -0.47 & -0.53 & -0.02 & -0.03 & -0.01 & -0.01 & 0.00 \\
\hline Togo & 10,200 & 1.75 & 172 & 0.30 & 1.48 & 0.07 & 0.10 & 2.18 & 0.26 & 0.67 \\
\hline Nepal & 11,900 & 1.49 & 125 & 0.19 & 1.08 & 0.05 & 0.07 & 0.39 & 0.27 & 0.11 \\
\hline Gambia & 7,500 & 1.47 & 197 & 0.27 & 1.30 & 0.06 & 0.08 & 6.07 & 0.57 & 1.29 \\
\hline Iraq & 57,200 & 1.39 & 24 & -0.69 & -1.40 & -0.06 & -0.09 & -0.04 & -0.01 & -0.01 \\
\hline Greece & 11,200 & 1.29 & 115 & 0.15 & 0.89 & 0.04 & 0.06 & 0.02 & 0.01 & 0.01 \\
\hline Slovenia & 5,200 & 0.79 & 152 & 0.12 & 0.63 & 0.03 & 0.04 & 0.05 & 0.04 & 0.02 \\
\hline Timor-Leste & 2,000 & 0.45 & 224 & 0.09 & 0.41 & 0.02 & 0.03 & 6.05 & 1.03 & 0.48 \\
\hline Albania & 3,200 & 0.36 & 113 & 0.04 & 0.25 & 0.01 & 0.02 & 0.05 & 0.07 & 0.02 \\
\hline Oman & 15,000 & 0.30 & 20 & -0.20 & -0.44 & -0.02 & -0.03 & -0.03 & -0.01 & -0.02 \\
\hline Bhutan & 4,300 & 0.30 & 69 & 0.00 & 0.12 & 0.01 & 0.01 & 0.07 & 0.26 & 0.10 \\
\hline
\end{tabular}




\begin{tabular}{|c|c|c|c|c|c|c|c|c|c|c|}
\hline Israel & 2,400 & 0.09 & 38 & -0.02 & -0.02 & 0.00 & 0.00 & 0.00 & 0.00 & 0.00 \\
\hline Eq. Guinea & 100 & 0.04 & 412 & 0.01 & 0.04 & 0.00 & 0.00 & 0.47 & 0.06 & 0.02 \\
\hline Saudi Arabia & 2,800 & 0.04 & 13 & -0.04 & -0.11 & 0.00 & -0.01 & 0.00 & 0.00 & 0.00 \\
\hline
\end{tabular}

Table 5.1. Potential energy production of global pastures by country, ranked highest to lowest by biomass production. The total area of harvestable pastures equals total area of $100 \mathrm{~km}^{2}$ cells with $\geq 25 \%$ pasture coverage. Harvestable biomass equals $50 \%$ of estimated ANPP. Ethanol is total produced via cellulosic methods $(0.255 \mathrm{~L} / \mathrm{kg}$ dry weight $)$ less energy required to harvest, transport and process biomass. Electricity, gasoline, and diesel are amounts co-produced via IGCC-FT processing less energy required to harvest, transport and process biomass. Total energy produced via IGCC-FT is $48.75 \%$ higher than cellulosic ethanol, and products are divided into electricity (47\%) and liquid fuels (53\%), the latter further divided into diesel $(53 \%)$ and gasoline $(47 \%)$. The ratios of potential electricity, gasoline, and diesel produced via IGCC-FT processing to their respective in-country demands. 


\begin{tabular}{rlclclr} 
Country & $\begin{array}{c}\text { Harvestable } \\
\text { Area } \\
\left(\mathbf{k m}^{2}\right)\end{array}$ & Country & $\begin{array}{c}\text { Harvestable } \\
\text { Biomass } \\
\text { (tons) }\end{array}$ & Country & $\begin{array}{c}\text { Yield } \\
\text { (tons/km }^{2} \text { ) }\end{array}$ \\
\hline 1 & China & $4,871,500$ & Brazil & $1,349,475,136$ & Uruguay & 573 \\
2 & Australia & $4,349,500$ & China & $588,140,848$ & Paraguay & 530 \\
3 & Kazakhstan & $3,821,000$ & Australia & $545,861,542$ & Mozambique & 454 \\
4 & Brazil & $3,239,700$ & United States & $477,688,372$ & Bolivia & 421 \\
5 & United States & $2,498,600$ & Angola & $326,627,356$ & Tanzania & 418 \\
6 & Russia & $2,000,600$ & Sudan & $290,168,789$ & Colombia & 417 \\
7 & Argentina & $1,875,400$ & Argentina & $279,285,660$ & Brazil & 417 \\
8 & Mongolia & $1,592,100$ & Mozambique & $251,199,400$ & Dom. Republic & 415 \\
9 & Sudan & $1,575,300$ & Colombia & $237,642,698$ & Equatorial Guinea & 412 \\
10 & South Africa & $1,242,500$ & Bolivia & $218,791,220$ & Nicaragua & 412 \\
11 & Iran & $1,090,100$ & Tanzania & $204,689,280$ & Angola & 400 \\
12 & Mexico & $1,084,500$ & South Africa & $201,976,786$ & Swaziland & 390 \\
13 & Angola & 816,000 & Kazakhstan & $190,266,770$ & Zambia & 381 \\
14 & Colombia & 570,200 & Paraguay & $182,971,927$ & Venezuela & 357 \\
15 & Chad & 570,000 & Mexico & $151,265,707$ & Rwanda & 357 \\
16 & Mozambique & 552,800 & Zambia & $135,744,672$ & Uganda & 353 \\
17 & Namibia & 544,500 & Uruguay & $133,758,904$ & Côte d'Ivoire & 348 \\
18 & Mali & 539,600 & Russia & $128,581,175$ & Costa Rica & 345 \\
19 & Somalia & 526,800 & Madagascar & $122,966,751$ & El Salvador & 339 \\
20 & Bolivia & 520,000 & Mongolia & $120,203,007$ & Madagascar & 333 \\
21 & Tanzania & 489,400 & Venezuela & $97,902,316$ & Congo & 321 \\
22 & Canada & 484,100 & Chad & $94,600,683$ & Cuba & 320 \\
23 & Algeria & 472,500 & Congo & $72,163,749$ & Guatemala & 318
\end{tabular}


24 Turkmenistan

25 Uzbekistan
448,700

395,100

\section{Zimbabwe}

New Zealand
$60,396,014$

$55,638,155$
Guinea

Malawi
315

307

Table 5.2. The top 25 countries for harvestable area, harvestable biomass, and mean yield of biomass. Harvestable biomass is $50 \%$ of total ANPP, leaving $50 \%$ of production for habitat, soil building and carbon sequestration. 


\begin{tabular}{|c|c|c|c|c|c|c|c|c|}
\hline & $\begin{array}{c}\text { Area } \\
(\mathbf{k m} 2)\end{array}$ & $\begin{array}{c}\text { FT Net } \\
\text { Energy } \\
\text { Output } \\
\text { (GJ) }\end{array}$ & $\begin{array}{c}\text { FT } \\
\text { Electrcity } \\
\text { (million } \\
\text { MwH) }\end{array}$ & $\begin{array}{c}\text { FT } \\
\text { Gasoline } \\
\text { (billion L) }\end{array}$ & $\begin{array}{l}\text { FT Diesel } \\
\text { (billion L) }\end{array}$ & $\begin{array}{c}\text { FT } \\
\text { Electricity } \\
\text { Prod/Use }\end{array}$ & $\begin{array}{c}\text { FT } \\
\text { Gasoline } \\
\text { Prod/Use }\end{array}$ & $\begin{array}{c}\text { FT } \\
\text { Diesel } \\
\text { Prod/Use }\end{array}$ \\
\hline \multicolumn{9}{|l|}{ United States } \\
\hline Corn Feedcrop & 148,511 & 0.6 & 82.60 & 5.37 & 3.66 & 0.02 & 0.01 & 0.02 \\
\hline Soy Feedcrop & 215,590 & 0.9 & 119.90 & 7.80 & 5.31 & 0.03 & 0.01 & 0.04 \\
\hline Wheat Feedcrop & 27,757 & 0.1 & 15.44 & 1.00 & 0.68 & 0.00 & 0.00 & 0.00 \\
\hline Total Feedcrop Area & 391,857 & 1.6 & 217.94 & 14.17 & 9.66 & 0.05 & 0.02 & 0.06 \\
\hline Corn Ethanol & 159,119 & 0.7 & 88.50 & 5.75 & 3.92 & 0.02 & 0.01 & 0.03 \\
\hline Total Crop Area & 550,977 & 2.3 & 306.43 & 19.93 & 13.58 & 0.07 & 0.03 & 0.09 \\
\hline Pasture & $2,498,600$ & 3.2 & 417.49 & 27.13 & 18.49 & 0.11 & 0.04 & 0.12 \\
\hline Total & $3,049,577$ & 5.5 & $\begin{array}{r}723.93 \\
0.00\end{array}$ & 47.05 & 32.07 & 0.18 & 0.07 & 0.21 \\
\hline Corn Feedcrop & 64,017 & 0.3 & 118.56 & 7.71 & 5.25 & 0.26 & 0.23 & 0.16 \\
\hline Soy Feedcrop & 174,565 & 1.0 & 323.30 & 21.02 & 14.33 & 0.71 & 0.63 & 0.42 \\
\hline Total Feedcrop Area & 238,582 & 1.3 & 441.86 & 28.73 & 19.58 & 0.97 & 0.86 & 0.58 \\
\hline Sugarcane Ethanol & 0 & 0.5 & 95.82 & 6.23 & 4.25 & 0.21 & 0.19 & 0.13 \\
\hline Total Crop Area & 238,582 & 1.8 & 537.68 & 34.96 & 23.83 & 1.18 & 1.05 & 0.71 \\
\hline Pasture & $3,239,700$ & 10.6 & $1,443.39$ & 93.79 & 63.92 & 3.03 & 2.68 & 1.81 \\
\hline Total & $3,478,282$ & 12.4 & 1,981.07 & 128.75 & 87.75 & 4.21 & 3.73 & 2.52 \\
\hline
\end{tabular}

Table 5.3. Potential energy production via IGCC-FT from croplands and pasture in the U.S. and Brazil. Electricity, gasoline, and diesel are amounts co-produced via IGCC-FT processing less energy required to harvest, transport and process biomass. 
Products are divided into electricity (47\%) and liquid fuels (53\%), the latter further divided into diesel (53\%) and gasoline (47\%). The ratios are of potential electricity, gasoline, and diesel produced via IGCC-FT processing to their respective incountry demands. 


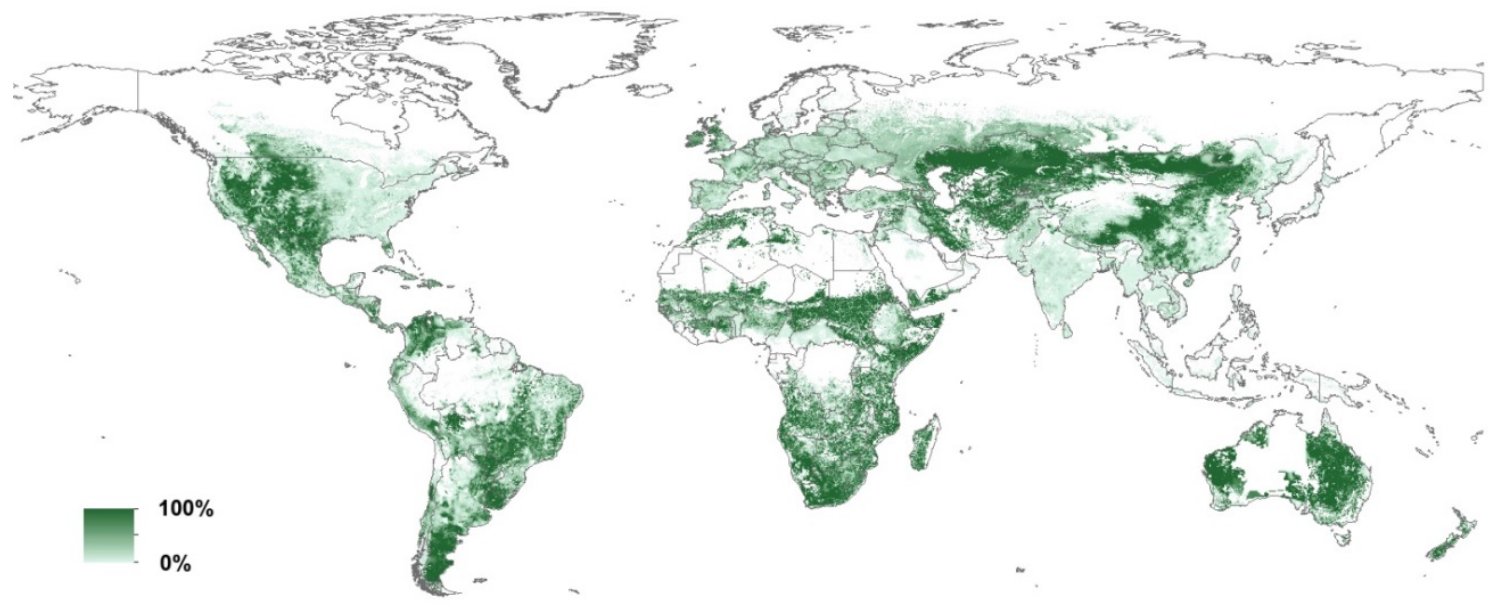

Fig 5.1. Map of global distribution of pastures. Values are percent cover of $\sim 100 \mathrm{~km}^{2}$ cells. 


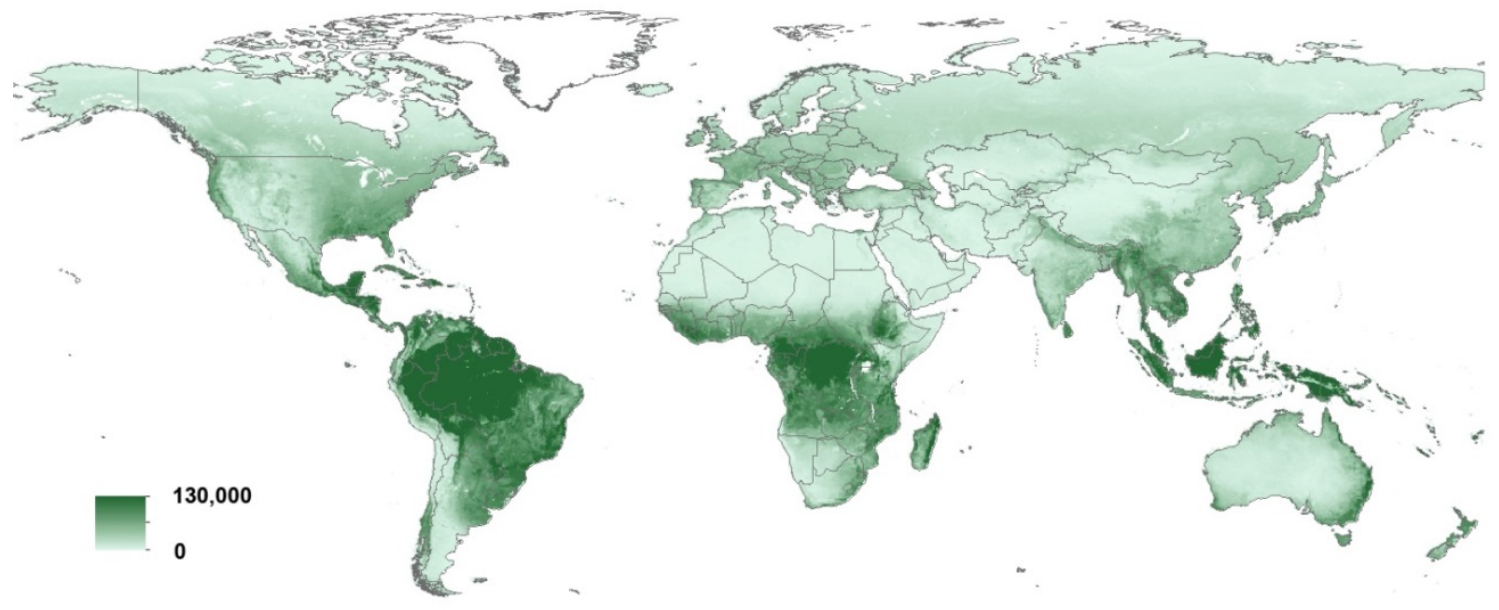

Fig 5.2. Map of global NPP. Values are tons $\mathrm{C} / 100 \mathrm{~km}^{2}$. Maximum value is $128,549.8$ tons $\mathrm{C} / 100 \mathrm{~km}^{2}$. Mean value is $25,849.3$ tons $\mathrm{C} / 100 \mathrm{~km}^{2}$. 

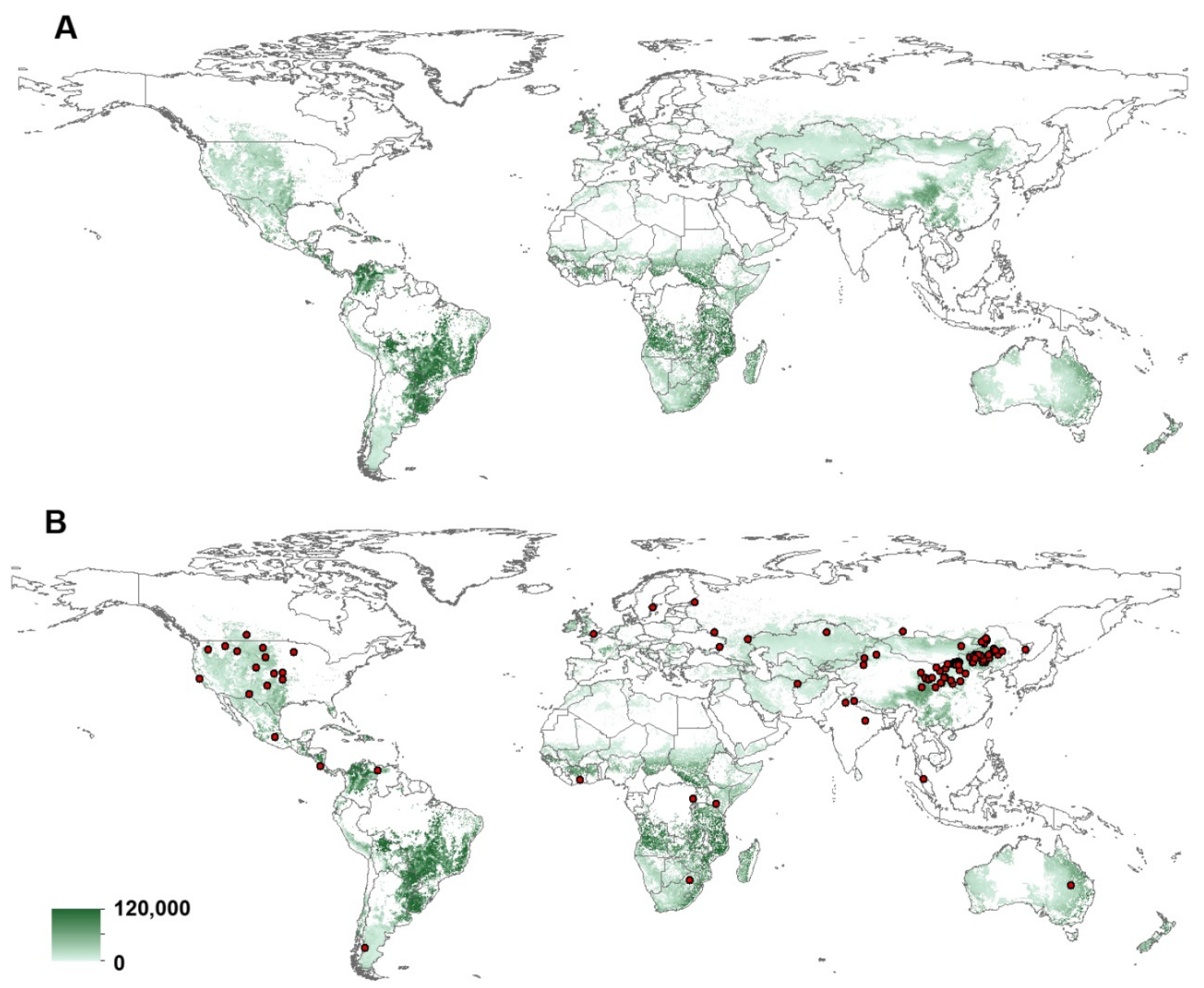

Fig. 5.3. Map of global distribution of harvestable pastures. (a) NPP of pastures. Values are tons $\mathrm{C} / 100 \mathrm{~km}^{2}$. Maximum value is $118,455.7$ tons $\mathrm{C} / 100 \mathrm{~km}^{2}$. Mean value is 165.3 tons $\mathrm{C} / 100 \mathrm{~km}^{2}$. (b) Locations of sample sites for comparison of Pasture Map NPP values to literature values. 


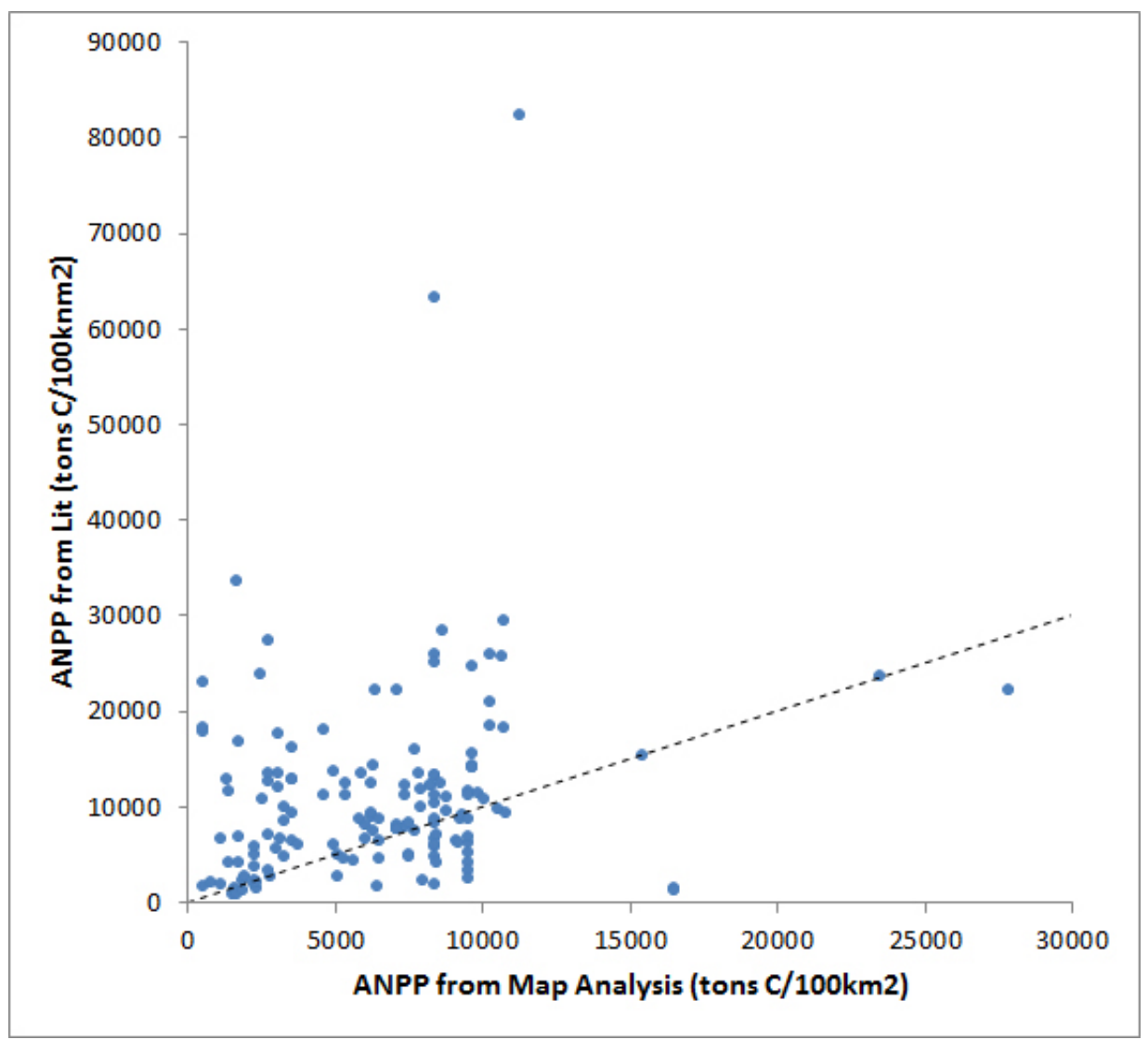

Fig. 5.4. Relationship between ANPP estimated from the map analysis and published ANPP values at 146 sample locations of pastures. The dotted line is the 1:1 ratio. ANPP is higher from literature values than map analysis at 104 locations ( $71 \%$ of the sites). 

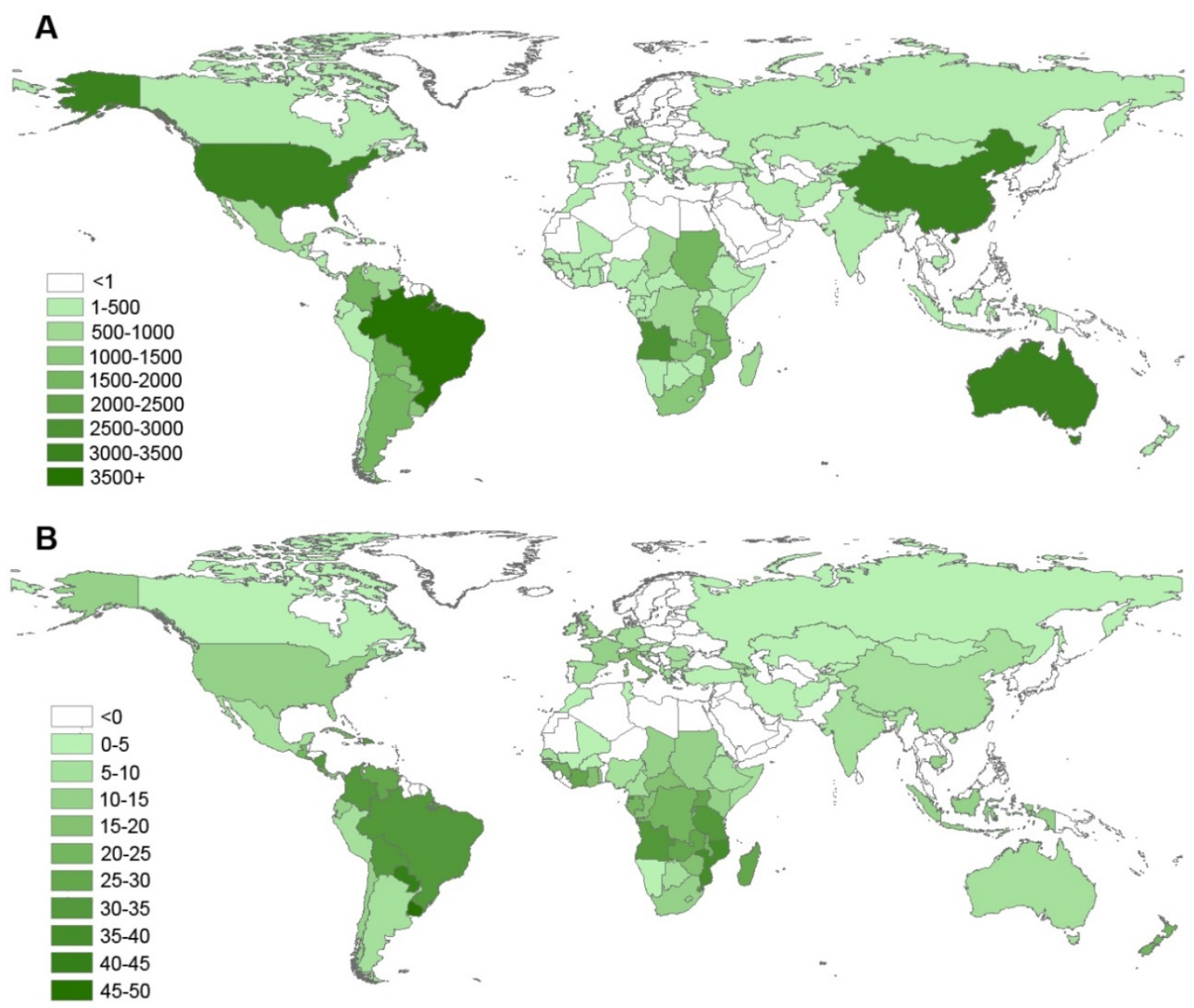

Fig. 5.5. Potential energy output from pastures via IGCC-FT processing less energy required to harvest, transport and process biomass. (a) Total potential energy. Values are in billion megajoules. (b) Average yield. Values are in gigajoules/ha. 

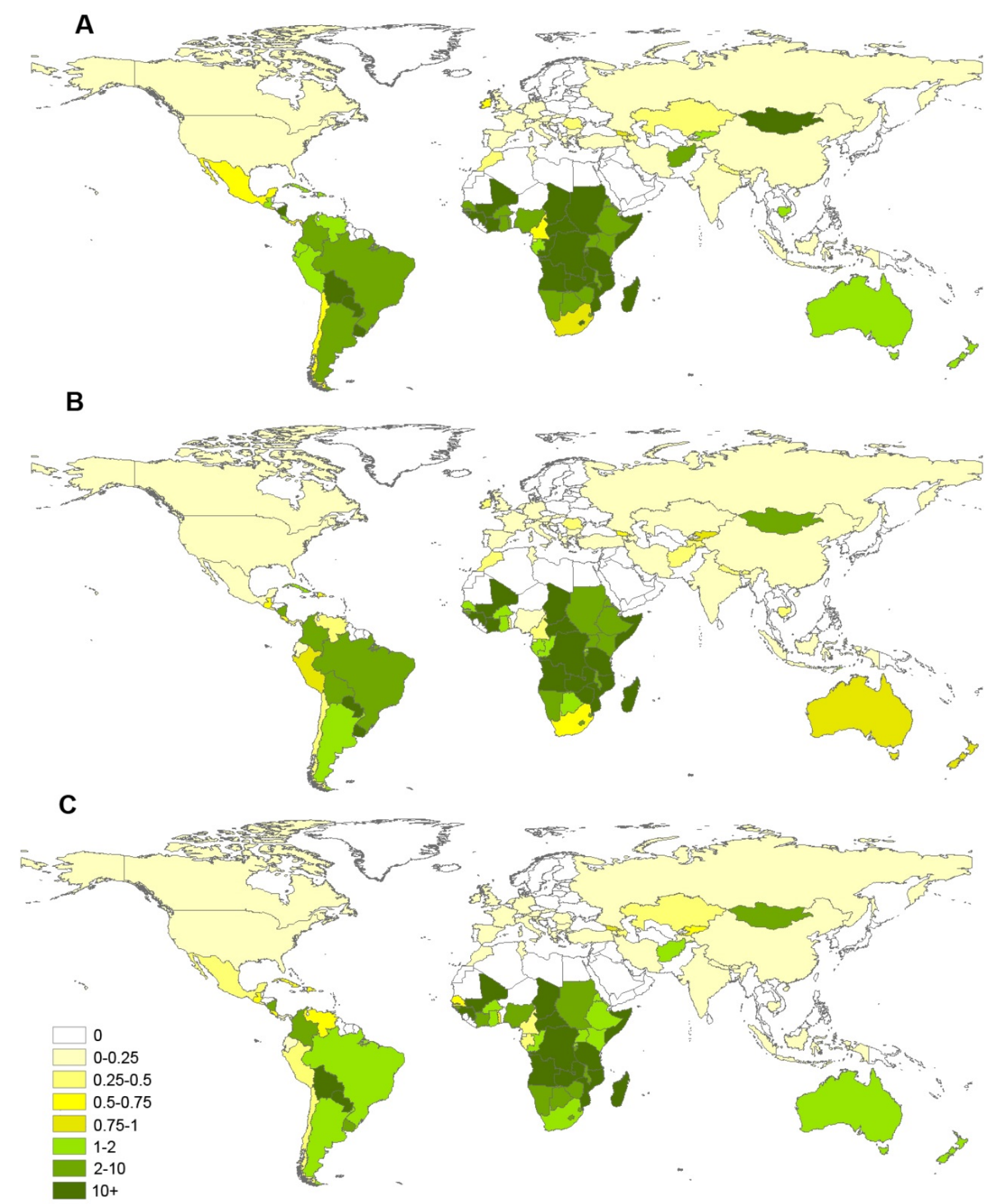

Fig. 5.6. Ratios of potential energy production of pastures from IGCC-FT processing vs. internal demand for (a) electricity, (b) gasoline, and (c) diesel. 


\section{Literature Cited}

Administration, U.S.E.I., 2012. International Energy Statistics. U.S. Department of Energy, Washington, D.C.

Advisor, S.a.C., 2014. soybeansandcorn.com, Hinsdale, IL.

Ajanovic, A., 2011. Biofuels versus food production: Does biofuels production increase food prices? Energy 36, 2070-2076.

Alexandratos, N., Bruinsma, J., 2012. World agriculture towards 2030/2050: the 2012 revision. FAO, Rome.

Association, B.S.I., 2015. UNICA.

Association, T.A.S., 2014. SoyStats 2014.

Baka, J., Roland-Holst, D., 2009. Food or fuel? What European farmers can contribute to Europe's transport energy requirements and the Doha Round. Energy Policy 37, 25052513.

Birt, N., 2012. Wheat for livestock feed estimated to rise sharply, AgWeb. Farm Journal.

Cassidy, E., West, P.C., Gerber, J.S., Foley, J.A., 2013. Redefining agricultural yields: from tonnes to people nourished per hectare. Environmental Research Letters 8, 8.

Change, I.P.O.C., 2007. Report of The Nineteenth Session of the Intergovernmental Panel on Climate Change (IPCC) Geneva, 17-20 (am only) April 2002.

Charles, M.B., Ryan, R., Ryan, N., Oloruntoba, R., 2007. Public policy and biofuels: The way forward? Energy Policy 35, 5737-5746.

De Oliveira, M.E.D., Vaughan, B.E., Rykiel, E.J., 2005. Ethanol as fuel: energy, carbon dioxide balances, and ecological footprint. Bioscience 55, 593-602.

Demirbas, A., 2008. Biofuels sources, biofuel policy, biofuel economy and global biofuel projections. Energy Conversion and Management 49, 2106-2116.

Demirbas, A., 2009. Biorefineries: Current activities and future developments. Energy Conversion and Management 50, 2782-2801.

Dry, M.E., 2002. The Fischer-Tropsch process: 1950-2000. Catalysis Today 71, 227241.

Duke, C.S., Pouyat, R.V., Robertson, G.P., Parton, W.J., 2013. Ecological Dimensions of Biofuels. . Issues in Ecology Report No. 17.

Escobar, J.C., Lora, E.S., Venturini, O.J., Yáñez, E.E., Castillo, E.F., Almazan, O., 2009. Biofuels: Environment, technology and food security. Renewable and Sustainable Energy Reviews 13, 1275-1287. 
ESRI, 2011. ArcGIS Desktop, Release 10. . Environmental Systems Research Institute, Redlands, CA.

Foley, J.A., DeFries, R., Asner, G.P., Barford, C., Bonan, G., Carpenter, S.R., Chapin, F.S., Coe, M.T., Daily, G.C., Gibbs, H.K., Helkowski, J.H., Holloway, T., Howard, E.A., Kucharik, C.J., Monfreda, C., Patz, J.A., Prentice, I.C., Ramankutty, N., Snyder, P.K., 2005. Global Consequences of Land Use. Science 309, 570-574.

Foley, J.A., Ramankutty, N., Brauman, K.A., Cassidy, E.S., Gerber, J.S., Johnston, M., Mueller, N.D., O/'Connell, C., Ray, D.K., West, P.C., Balzer, C., Bennett, E.M., Carpenter, S.R., Hill, J., Monfreda, C., Polasky, S., Rockstrom, J., Sheehan, J., Siebert, S., Tilman, D., Zaks, D.P.M., 2011. Solutions for a cultivated planet. Nature 478, 337342.

Hannula, I., Kurkela, E., 2013. Liquid transportation fuels via large-scale fluidised-bed gasification of lignocellulosic biomass.

Harvey, M., Pilgrim, S., 2011. The new competition for land: Food, energy, and climate change. Food Policy 36, Supplement 1, S40-S51.

Havlík, P., Schneider, U.A., Schmid, E., Böttcher, H., Fritz, S., Skalský, R., Aoki, K., Cara, S.D., Kindermann, G., Kraxner, F., Leduc, S., McCallum, I., Mosnier, A., Sauer, T., Obersteiner, M., 2011. Global land-use implications of first and second generation biofuel targets. Energy Policy 39, 5690-5702.

Hubert, B., Rosegrant, M., van Boekel, M.A., Ortiz, R., 2010. The future of food: scenarios for 2050. Crop Sci. 50, S-33-S-50.

Hui, D., Jackson, R.B., 2006. Geographical and interannual variability in biomass partitioning in grassland ecosystems: a synthesis of field data. New Phytol. 169, 85-93.

Ibrahim, M.A., 'T Mannetje, L., 1998. Compatibility, persistence and productivity of grass-legume mixtures in the humid tropics of Costa Rica. 1. Dry matter yield, nitrogen yield and botanical composition. Tropical Grasslands 32, 96-104.

IEA/AMF, 2007. Synthetic gasoline and diesel oil produced by Fischer-Tropsch Technology. A possibility for the future? International Energy Agency's (IEA) Implementing Agreement on Advanced Motor Fuels (IEA/AMF), Göteborg, Sweden.

Imhoff, M.L., Bounoua, L., Ricketts, T., Loucks, C., Harriss, R., Lawrence, W.T., 2004. HANPP Collection: Global Patterns in Net Primary Productivity (NPP). NASA Socioeconomic Data and Applications Center (SEDAC), Palisades, NY.

Irisarri, J.G.N., Oesterheld, M., Golluscio, R.A., Paruelo, J.M., 2014. Effects of Animal Husbandry on Secondary Production and Trophic Efficiency at a Regional Scale. Ecosystems 17, 738-749. 
Keating, B., Verburg, K., Huth, N., Robertson, M., 1997. Nitrogen management in intensive agriculture: sugarcane in Australia.

Krausmann, F., Erb, K.-H., Gingrich, S., Lauk, C., Haberl, H., 2008. Global patterns of socioeconomic biomass flows in the year 2000: A comprehensive assessment of supply, consumption and constraints. Ecol. Econ. 65, 471-487.

Lawler, J.J., Shafer, S.L., White, D., Kareiva, P., Maurer, E.P., Blaustein, A.R., Bartlein, P.J., 2009. Projected climate-induced faunal change in the Western Hemisphere. Ecology 90, 588-597.

Machado, I.M., Atsumi, S., 2012. Cyanobacterial biofuel production. J. Biotechnol. 162, $50-56$.

Machovina, B., Feeley, K.J., 2014a. Livestock: limit red meat consumption. Nature 508, 186-186.

Machovina, B., Feeley, K.J., 2014b. Meat consumption as a key impact on tropical nature: a response to Laurance et. al. Trends Ecol. Evol. 29, 430.

Machovina, B., Feeley, K.J., 2014c. Taking a Bite Out of Biodiversity. Science 343, 838.

Mathews, J.A., 2008. Carbon-negative biofuels. Energy Policy 36, 940-945.

McCarty, J.P., 2001. Ecological Consequences of Recent Climate Change. Conserv. Biol. $15,320-331$.

McMichael, A.J., Powles, J.W., Butler, C.D., Uauy, R., 2007. Food, livestock production, energy, climate change, and health. The Lancet 370, 1253-1263.

Mendoza, T., Rosario, E., Pizon, E., 1980. Drymatter yield and feeding value of sugarcane as influenced by spacing and age of cutting. Philippine Journal of Crop Science 5, 34-36.

Nellemann, C., 2009. The Environmental Food Crisis: The Environment's Role in Averting Future Food Crises: a UNEP Rapid Response Assessment. UNEP/Earthprint.

Nigam, P.S., Singh, A., 2011. Production of liquid biofuels from renewable resources. Progress in Energy and Combustion Science 37, 52-68.

Pelletier, N., Tyedmers, P., 2010. Forecasting potential global environmental costs of livestock production 2000-2050. Proceedings of the National Academy of Sciences 107, 18371-18374.

Pimentel, D., Patzek, T.W., 2008. Ethanol production: energy and economic issues related to US and Brazilian sugarcane, Biofuels, Solar and Wind as Renewable Energy Systems. Springer, pp. 357-371. 
Potter, C.S., Randerson, J.T., Field, C.B., Matson, P.A., Vitousek, P.M., Mooney, H.A., Klooster, S.A., 1993. Terrestrial ecosystem production: a process model based on global satellite and surface data. Global Biogeochemical Cycles 7, 811-841.

Ramankutty, N., Evan, A.T., Monfreda, C., Foley, J.A., 2000. Global Agricultural Lands: Pastures, 2000. NASA Socioeconomic Data and Applications Center (SEDAC), Palisades, NY.

Rathmann, R., Szklo, A., Schaeffer, R., 2010. Land use competition for production of food and liquid biofuels: An analysis of the arguments in the current debate. Renewable Energy 35, 14-22.

Reijnders, L., Soret, S., 2003. Quantification of the environmental impact of different dietary protein choices. The American Journal of Clinical Nutrition 78, 664S-668S.

Ripple, W.J., Smith, P., Haberl, H., Montzka, S.A., McAlpine, C., Boucher, D.H., 2014. Ruminants, climate change and climate policy. Nature Clim. Change 4, 2-5.

Robertson, D.E., Jacobson, S.A., Morgan, F., Berry, D., Church, G.M., Afeyan, N.B., 2011. A new dawn for industrial photosynthesis. Photosynthesis Res. 107, 269-277.

Rosenzweig, C., Parry, M.L., 1994. Potential impact of climate change on world food supply. Nature 367, 133-138.

Schirmer, A., Rude, M.A., Li, X., Popova, E., Del Cardayre, S.B., 2010. Microbial biosynthesis of alkanes. Science 329, 559-562.

Scurlock, J.M.O., Johnson, K., Olson, R.J., 2002. Estimating net primary productivity from grassland biomass dynamics measurements. Global Change Biol. 8, 736-753.

Steinfeld, H., Gerber, P., Wassenaar, T.D., Castel, V., Haan, C.d., 2006. Livestock's Long Shadow: Environmental Issues and Options. Food and Agriculture Organization of the United Nations, Rome.

Taheripour, F., Hertel, T.W., Tyner, W.E., 2011. Implications of biofuels mandates for the global livestock industry: a computable general equilibrium analysis. Agricultural Economics 42, 325-342.

Takeshita, T., Yamaji, K., 2008. Important roles of Fischer-Tropsch synfuels in the global energy future. Energy Policy 36, 2773-2784.

Tilman, D., Fargione, J., Wolff, B., D'Antonio, C., Dobson, A., Howarth, R., Schindler, D., Schlesinger, W.H., Simberloff, D., Swackhamer, D., 2001. Forecasting Agriculturally Driven Global Environmental Change. Science 292, 281-284.

Tilman, D., Hill, J., Lehman, C., 2006. Carbon-Negative Biofuels from Low-Input HighDiversity Grassland Biomass. Science 314, 1598-1600. 
Tirado, M.C., Cohen, M.J., Aberman, N., Meerman, J., Thompson, B., 2010. Addressing the challenges of climate change and biofuel production for food and nutrition security. Food Res. Int. 43, 1729-1744.

USDA, E.R.S., 2014. U.S. Feedgrain Production 2014/15.

Walther, G.-R., Post, E., Convey, P., Menzel, A., Parmesan, C., Beebee, T.J., Fromentin, J.-M., Hoegh-Guldberg, O., Bairlein, F., 2002. Ecological responses to recent climate change. Nature 416, 389-395.

Wilson, J.B., Peet, R.K., Dengler, J., Pärtel, M., 2012. Plant species richness: the world records. Journal of Vegetation Science 23, 796-802. 
VITA

\section{BRIAN LOUIS MACHOVINA}

Born, Elyria, Ohio

1992

B.S., Biology

Florida International University

Miami, FL

1994

M.S., Biology

Florida International University

Miami, FL

Ph.D. Program, Biology; Reseach Assistant, SERP

Florida International University

Miami, FL

1997-2000

Assistant Director

Oasis Preserve International

Los Angeles, CA

2000-2002

Director of Business Development

Guayaki Sustainable Rainforest Products, Inc.

Los Angeles, CA

2002-2004

Executive Director

California Coastkeeper Alliance

Los Angeles, CA

2004-2005

Vice President \& CFO

Essential Living Foods, Inc.

San Luis Obispo, CA

2006-2010

Co-Owner

Essential Living Foods, Inc.

San Luis Obispo, CA

2010-2015

Doctoral Candidate

Florida International University

Miami, Florida

Co-Founder 
Healthy Foods, LLC

Cleveland, $\mathrm{OH}$

\section{PUBLICATIONS}

Machovina, B. (2015) Eating plants to save them. Proceedings of "Botanists of the 21st century: roles, challenges and opportunities," UNESCO, Paris, 22-25 September 2014.

Machovina, B. \& K. Feeley (2014) Meat consumption's impact on tropics: a key challenge. Trends in Ecology \& Evolution. 29(8):430.

Machovina, B. \& K. Feeley (2014) Livestock: limit red meat consumption. Nature 508:186

Machovina, B. \& K. Feeley (2014) Taking a bite out of biodiversity. Science 343(6173):838

Feeley, K. \& B. Machovina. (2014) Increasing preference for beef magnifies human impact on world's food web. Proceedings of the National Academy of Sciences 111(9), E794.

Machovina, B. \& K. Feeley (2013) Climate change driven shifts in the extent and location of areas suitable for export banana production. Journal of Ecological Economics 95:83-95.

Feeley, K.J., E. Rehm, \& B. Machovina. (2013) The responses of tropical forest species to global climate change: acclimate, adapt, migrate, or go extinct? Frontiers of Biogeography, 4(2). 